Estimação de obstáculos e área de pista com pontos 3D esparsos 

SERVIÇO DE PÓS-GRADUAÇÃO DO ICMC-USP

Data de Depósito:

Assinatura:

\title{
Estimação de obstáculos e área de pista com pontos 3D esparsos
}

\author{
Patrick Yuri Shinzato
}

Orientador: Prof. Dr. Denis Fernando Wolf

Tese apresentada ao Instituto de Ciências Matemáticas e de Computação - ICMC-USP, como parte dos requisitos para obtenção do título de Doutor em Ciências - Ciências de Computação e Matemática Computacional. VERSÃO REVISADA 
Ficha catalográfica elaborada pela Biblioteca Prof. Achille Bassi e Seção Técnica de Informática, ICMC/USP, com os dados fornecidos pelo(a) autor(a)

\begin{tabular}{|c|c|}
\hline \multirow[t]{3}{*}{$\mathrm{S} 556 \mathrm{e}$} & $\begin{array}{l}\text { Shinzato, Patrick Yuri } \\
\quad \text { Estimação de obstáculos e área de pista com } \\
\text { pontos 3D esparsos / Patrick Yuri Shinzato; } \\
\text { orientador Denis Fernando Wolf. -- São Carlos, } 2015 . \\
\quad 79 \text { p. }\end{array}$ \\
\hline & $\begin{array}{l}\text { Tese (Doutorado - Programa de Pós-Graduação em } \\
\text { Ciências de Computação e Matemática Computacional) -- } \\
\text { Instituto de Ciências Matemáticas e de Computação, } \\
\text { Universidade de São Paulo, } 2015 \text {. }\end{array}$ \\
\hline & $\begin{array}{l}\text { 1. Robótica Móvel. 2. Veículos Inteligentes. } 3 . \\
\text { Visão Computacional. 4. Câmera Estereoscópica. I. } \\
\text { Wolf, Denis Fernando, orient. II. Título. }\end{array}$ \\
\hline
\end{tabular}


Estimation of obstacles and road area with sparse 3D points 

SERVIÇO DE PÓS-GRADUAÇÃO DO ICMC-USP

Data de Depósito:

Assinatura:

\title{
Estimation of obstacles and road area with sparse 3D points
}

\author{
Patrick Yuri Shinzato
}

Advisor: Prof. Dr. Denis Fernando Wolf

Doctoral dissertation submitted to the Instituto de Ciências Matemáticas e de Computação - ICMC-USP, in partial fulfillment of the requirements for the degree of the Doctorate Program in Computer Science and Computational Mathematics. FINAL VERSION.

USP - São Carlos

May 2015 
Ficha catalográfica elaborada pela Biblioteca Prof. Achille Bassi e Seção Técnica de Informática, ICMC/USP, com os dados fornecidos pelo(a) autor(a)

\begin{tabular}{|c|c|}
\hline \multirow[t]{3}{*}{ S556e } & $\begin{array}{l}\text { Shinzato, Patrick Yuri } \\
\text { Estimation of obstacles and road area with } \\
\text { sparse 3D points / Patrick Yuri Shinzato; } \\
\text { orientador Denis Fernando Wolf. -- São Carlos, } 2015 \text {. } \\
\quad 79 \text { p. }\end{array}$ \\
\hline & $\begin{array}{l}\text { Tese (Doutorado - Programa de Pós-Graduação em } \\
\text { Ciências de Computação e Matemática Computacional) -- } \\
\text { Instituto de Ciências Matemáticas e de Computação, } \\
\text { Universidade de São Paulo, } 2015 \text {. }\end{array}$ \\
\hline & $\begin{array}{l}\text { 1. Mobile robotics. 2. Intelligent vehicles. } 3 . \\
\text { Computer vision. 4. Stereo camera. I. Wolf, Denis } \\
\text { Fernando, orient. II. Título. }\end{array}$ \\
\hline
\end{tabular}




\section{Abstract}

Worldwide, an estimated 1.2 million lives are lost in road crashes each year and Advanced Driver Assistance Systems (ADAS) and Self-driving cars promise to reduce this number. Among the various issues to complete this technology, perception systems are still an unsolved issues. Normally two of them, obstacle detection and road detection, make use of sophisticated algorithms such as supervised machine learning methods which can perform with impressive results if it was trained with good datasets. Since it is a complex and an expensive job to create and maintain databases of scenarios from the entire world, adaptive and/or self-supervised methods are good candidates for detection systems in the near future. Due that, this thesis present a method to estimate obstacles and estimate the road terrain using low cost sensors (stereo camera), avoiding supervised machine learning techniques and the most common assumptions used by works presented in literature. These methods were compared with 3D-LIDAR approaches achieving similar results and thus it can be used as a pre-processing step to improve or allow adaptive methods with machine learning systems. 



\section{Resumo}

De acordo com a Organização Mundial da Saúde, cerca de 1,2 milhões de pessoas no mundo morrem em acidentes de trânsito. Sistemas de assistência ao motorista e veículos autônomos podem diminuir o número de acidentes. Dentre as várias demandas existentes para viabilizar essa tecnologia, sistemas computacionais de percepção ainda permanecem sem uma solução definitiva. Dois deles, detecção de obstáculos e de via navegável, normalmente fazem uso de algoritmos sofisticados como técnicas de aprendizado supervisionado, que mostram resultados impressionantes quando treinados com bases de dados bem definidas e diversificadas. Entretanto, construir, manter e atualizar uma base de dados com exemplos de vários lugares do mundo e em diversas situações é trabalhoso e complexo. Assim, métodos adaptativos e auto-supervisionados mostram-se como boas alternativas para sistemas de detecção do futuro próximo. Neste contexto, esta tese apresenta um método para estimar obstáculos e via navegável através de sensores de baixo custo (câmeras estereoscópicas), sem o uso de técnicas de aprendizado de máquina e de diversas suposições normalmente utilizadas por trabalhos ja disponíveis na literatura. Esses métodos utilizando sensor estereoscópico foram comparados fazendo uso de sensores do tipo 3D-LIDAR e mostraram resultados semelhantes. Este sistema poderá ser usado como uma fase pré-processamento de dados para melhorar ou viabilizar métodos adaptativos de aprendizado. 



\section{Acknowledgements Agradecimentos}

Eis que aqui se encontra o resultado de alguns anos de trabalho. Um resultado que significa o fim e o início de uma etapa da vida de todo aquele que procura contribuir de alguma forma para a ciência e benefício da sociedade. Por isso, não poderia deixar de colocar neste documento, o meu profundo agradecimento a todos que de alguma forma contribuíram para a execução deste trabalho. Neste sentido, agradeço ao meu orientador, o professor Denis Fernando Wolf, pela oportunidade da realização deste trabalho e principalmente pela amizade e confiança. Agradeço a toda a minha família pelo apoio. Agradeço aos meus pais, Cristina e Silvio Shinzato, pelo apoio e incentivo para concluir a faculdade, mestrado e agora o doutorado para enfim, seguir a carreira acadêmica. Agradeço a minha namorada Janaína, que vem me apoiando e tornando a minha vida muito mais feliz. Agradeço a minha irmã Marjolly e ao meu cunhado Leandro pela convivência desde o mestrado, ajudando a tornar São Carlos um bom lar. Agradeço à todos os amigos de São Carlos e Campo Grande pelo companheirismo, festas, reuniões e as várias conversas jogadas fora que ajudaram a passar o tempo de forma muito mais agradável. Eu não conseguirei listar todos aqui, mas eu não poderia deixar de agradecer as meninas (Taís, Drica e Line + Eloá) pelas inúmeras bagunças e viagens (aqui nas redondezas e na Europa). Um agradecimento especial a todos os meus amigos do laboratório que me aguentaram até agora, pois pego no pé da maioria deles, obrigado pela ajuda e convivência.

Agradecimentos ao Laboratório de Robótica Móvel e ICMC pela oportunidade e acolhimento para o desenvolvimento deste trabalho, ao Institut für Mess- und Regelungstechnik no KIT na Alemanha pelo período que trabalhei por lá. Agradecimento ao INCT-SEC, CNpQ e FAPESP que financiaram alguns dos projetos do LRM e finalmente agradecimentos à FAPESP processo: 2010/01305-1, pelo apoio financeiro da bolsa. 



\section{Contents}

Abstract

Resumo iii

$\begin{array}{ll}\text { Acknowledgements } & \text { v }\end{array}$

List of Figures $\quad$ ix

List of Tables $\quad$ xiii

1 Introduction 1

1.1 Problem Statement and Objectives . . . . . . . . . . . . . . . 4

1.2 Thesis Contributions . . . . . . . . . . . . . . . . 5

1.3 Applications ........................ 6

1.4 Thesis Outline . . . . . . . . . . . . . . . . . . . 6

$\begin{array}{lll}2 & \text { Related Work } & 7\end{array}$

3 Obstacle Estimation $\quad 11$

3.1 Definition of Obstacle by 3D Spatial-Condition . . . . . . . . . . . . 11

3.2 General Model of 3D Point Clouds in Screen Plane . . . . . . . . . . . . . . . 13

3.3 3D LIDAR + Monocular Image Sensor . . . . . . . . . . . . . . . . 15

3.4 Stereo Image Sensor . . . . . . . . . . . . . . . . . . . . . 16

3.5 Estimation of Obstacles in Image Plane . . . . . . . . . . . . . . . . 18

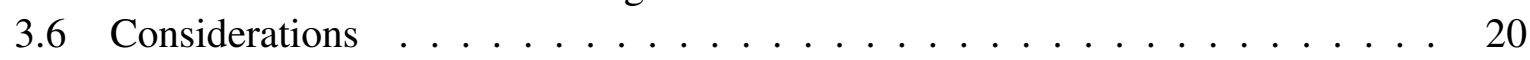


4 Road Estimation $\quad 21$

4.1 Perspective Modelling of Road Detection Problem . . . . . . . . . . . . 21

4.1.1 Polygon Generation . . . . . . . . . . . . . . . . 22

4.1.2 Estimation of Road Area . . . . . . . . . . . . . . . . . 22

4.2 Improvements with Green-Confidence . . . . . . . . . . . . . . . . . . 25

4.3 Considerations . . . . . . . . . . . . . . . . 28

5 Experimental Evaluation $\quad 29$

5.1 Online KITTI-ROAD Benchmark . . . . . . . . . . . . . . . . . . . . . . . . 32

5.2 Ground-Truth Images From training-dataset . . . . . . . . . . . . . . . . . 36

5.3 3D LIDAR + Monocular Image Sensor Approach . . . . . . . . . . . . . . . . . 38

5.4 Stereo Image Sensor Approach with Sparse-ELAS . . . . . . . . . . . . . . . 41

5.5 Stereo Image Sensor Approach with Semi-Global Block-Matching . . . . . . . 46

5.6 Considerations . . . . . . . . . . . . . . . . . . 47

6 Other Contributions and Applications 49

6.1 Road Markings Enhancement . . . . . . . . . . . . . . . . . . . . . . . 49

6.2 Lane Departure Warning . . . . . . . . . . . . . . . . . . . . 50

6.3 Automatic Semantic Waypoint Mapping . . . . . . . . . . . . . . . . 54

6.4 Navigation and Adaptive Cruise Control . . . . . . . . . . . . . . . . . . 57

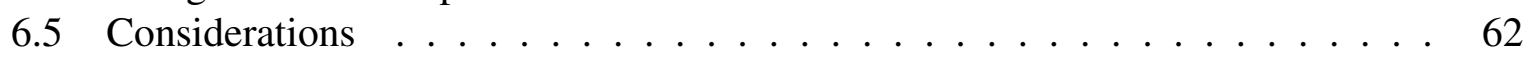

7 Conclusion $\quad 63$

7.1 Future Directions . . . . . . . . . . . . . . . . . . 64

$\begin{array}{ll}\text { Bibliography } & 67\end{array}$

$\begin{array}{ll}\text { A Published Papers } & \mathbf{7 7}\end{array}$

A.1 Journal Papers . . . . . . . . . . . . . . . . . . . . . . . . . . . . . . . . . . . . . . .

A.2 Conference Papers . . . . . . . . . . . . . . . 78 


\section{List of Figures}

1.1 The vehicular robotic platforms of CaRINA project. . . . . . . . . . . . . 3

1.2 Given a single 3D point cloud read from a sensors system installed in a vehicle platform and a screen projection function, the present work tries to detect the road terrain in the screen plane. (Images from KITTI-ROAD Benchmark dataset). 4

3.1 Intuition behind the algorithm from (Matthies et al., 1995) and (Talukder et al., 2002). The blue lines represents the laser projection of a lidar sensor. The higher patch with $H_{1}$ would probably be considered obstacle, while the lower patch with $\mathrm{H}_{2}$ would be considered traversable. Image adapted from (Talukder et al., 2002) . . . . . . . . . . . . . . . . . . . 12

3.2 The sigmoid-like threshold function is used to map an arbitrary value calculated between a pair of points for the [0,1] interval depending on parameters $\mu$ and $\sigma$.

3.3 Generic Modelling of 3D point clouds in screen plane. The cylinder of left image represents a 3D-lidar in the 3D world, in this work also represents the origin point $(x=0, y=0, z=0)$. The right image shows a screen plane (image world) with all points from 3D-lidar projected inside it. . . . . . . . . 14

3.4 Situations where the model cannot use structured neighbourhood. . . . . . . . 15

3.5 Upper image: 3D points are showed in red using the $(u, v)$ image coordinates obtained by transformation from matrices calibrations. Bottom image: Point cloud where each point is coloured (gray-scale) with the respectively pixel from the image. . . . . . . . . . . . . . . . . . . . . . 17

3.6 Results of reprojection step of a stereo image sensor. . . . . . . . . . . . . . 18

3.7 Sparse 3D points generated by stereo image sensors and projected in left image. 19

3.8 Results from our obstacle probability calculation method in a 2D image world. Warmer color pixels indicate more confidence level of this pixel being an obstacle. . . . . . . . . . . . . . . . . . . 20 
4.1 Comparison between the data structure of one polygon and a polar histogram that estimates road area. . . . . . . . . . . . . . . 23

4.2 Result of Estimation of road shape with several thresholds and origin points. . . 24

4.3 Result of our road estimation method as "estimation of each node $p$ of $G$ ". The $R(p)$ is propagate for all pixels neighbours of $p$ that are inside of an arbitrary radius of distance. . . . . . . . . . . . . . . . . . . . . 24

4.4 Out-city road without curbs but with grass as delimiter of the road area. . . . . 25

4.5 $\mathrm{L}^{*} \mathrm{a} * \mathrm{~b} *$ color space showing the $\left(\mathrm{a}^{*} \mathrm{~b} *\right)$-space with different $\mathrm{L}^{*}$ values. The darker colors are more likely to be blue than green/red. Coordinate $\mathrm{a}^{*}$ flows in the horizontal line, and coordinate $b^{*}$ in the vertical line. . . . . . . . . .

4.6 Result of our green detection method, warmer colors represents greater confidence about node $p$ be the grass that limits the road. Is possible to see that darker green does not have a good confidence since is too close to the limits of the upper left quadrant, i.e. too close of darker colors in the $\mathrm{L}^{*} \mathrm{a}^{*} \mathrm{~b}^{*}$ space. . . .

4.7 Result of combination of green-confidence with obstacle-confidence, warmer colors represents greater confidence about node $p$ be obstacle/grass that limits the road. Is easy to see a improvement in the grass area with good light conditions. 28

5.1 We adopted the same evaluation of (Fritsch et al., 2013) which evaluates the road area in a 2D metric space represented by bird-eye-view image (BEV). These samples of image results from a lane detection system shows the perspective effect that brings bias to pixels in near range (bottom part of image (a)), while in BEV images all pixels have the same weight in any classical pixel-based evaluation method. . . . . . . . . . . . . . .

5.2 The results in the first row belongs to GRES3D+VELO, while second contains results from GRES3D+SELAS and finally, the third row shows GRES3D+SGBM. A comparison between GRES3D+VELO and stereo approaches shows that 3D-LIDAR system has few threshold values that changes the recall range which means a more stable approach. Graphics available in KITTI website. . . . . . . . . . 34

5.3 Image results from all three approaches for the Urban Marked category. . . . . 35

5.4 Image results from all three approaches for the Urban Multiple Marked category. 35

5.5 Image results from all three approaches for the Urban Unmarked category. . . . 36

5.6 Example of ground-truth image that will degrade the performance of any ego-road estimation method, since it is not able to detect the side road. Upper Image: Original Image. Middle Image: Ground-truth from Kitti benchmark; green pixels represent the expected road area and red pixels represent no-road area. Bottom Image: Output from our method using a sensors system composed by a 3D-LIDAR and Monocular Camera. . . . . . . . . . . . . . . .

5.7 Given a ground-truth image (upper left image), the obstacle estimation method creates an obstacle-confidence map (upper right image) where lighter color represents more certainty about the pixel be an obstacle and darker pixels represents more certainty about the pixels be road. After that, the road estimation method generates the polygon that represents the road area, i.e. the green area from (bottom left image). . . . . . . . . . . . . . . . 
5.8 The MaxF and AP values (\% scores in $y$-axis) between these instances with different precision-degree's ( $x$-axis) show a nearly small linear variation between the results. Blue line represents MaxF and orange line represents AP.

5.9 Output from our proposed method using 3D-LIDAR sensor. From left to right, the first figure shows the original image, the middle shows the obstacle-confidence using 3D-LIDAR points, and last the third is the road-confidence in perspective view. All confidence-maps are shown in thermal-color style images, i.e. red color means the highest value while blue the lowest non-zero value, note that zero confidence means the original color (RGB).

5.10 Single-Frame Evaluation of GRES3D+VELO in Boxplot. It shows three sets of boxplots where each set shows four metric evaluations (Precision, Recall, FPR, FNR) for each category from training-dataset.

5.11 Worst result detected by the Boxplot representation. This image was highlighted as red ' + ' in category Urban Unmarked from the graphics displayed in Figure 5.10. This failure is caused by the non-existence of curbs or obstacles to delimit this road. . . . . . . . . . . . . . . . . . . .

5.12 Single-Frame Evaluation of GRES3D+SELAS in Boxplot. It shows three sets of boxplots where each set shows four metric evaluations (Precision, Recall, FPR, FNR) for each category from training-dataset.

5.13 Worst result detected by the Boxplot representation. This image was highlighted as red '+' in category Urban Unmarked from the graphics displayed in Figure 5.12. This failure is caused by noise in the disparity map which implies in a low confidence degree in the road area. After the selection of best-threshold which maximizes F1-measure, a huge amount of pixels (blue pixels) are classified as non-road. Despite the poor results generated by low confidence, you can see a result consistently classified. . . . . . . . . . . . . . . .

5.14 Good results of classification generated by GRES3D+SELAS showed in thermal color style, i.e. red means highest confidence degree about pixel be road and blue means lowest road-confidence that are greater than zero.

5.15 Samples of results from GRES3D+SGBM. It is possible see good detection in near range and failure in road markings edges. . . . . . . . . . . .

5.16 Single-Frame Evaluation of GRES3D+SGBM in Boxplot. It shows three sets of boxplots where each set shows four metric evaluations (Precision, Recall, FPR, FNR) for each category from training-dataset.

6.1 Each red box represents a pixel. The method calculates the difference of the value of the pixels in the same orientation (represented by the black lines) and distance from the target pixel (central box). Several orientations and distances are used for handling noise.

6.2 Upper image: shows the original image. Middle image: shows the same image after apply our method. Bottom image: shows the bird-eye-view image after apply the filter. Note that this filter is robust to shadows conditions. . . . . . . .

6.3 (a) ROI from the original image. (b) Image $M$ which has accumation from pixel's contrast. (c) Processed ROI image using a Otsu thresholding. . . . . . . . 52

6.4 Images result from ego-lane detetion system in normal conditions . . . . . . . 52 
6.5 Images result from system in night situations and strong shadows occurences

6.6 Levenberg-Marquardt fitting results. The orange and the magenta roundabout in 6.6a were collected at different times and have slightly different centers. In $6.6 \mathrm{~b}$ we perform the roundabout filtering to merge them into one Roundabout structure. . . . . . . . . . . . . . . . . .

6.7 Lane noise reduction. The lane points are shown in green and the red lines represent the calculated trajectory. . . . . . . . . . . . . . . . . 56

6.8 Campus II of USP Sao Carlos RVIZ view. The user can follow the 3D model of CaRINA II after selecting some point from our map. The current path is painted in purple. . . . . . . . . . . . . . . . . . . 57

6.9 Campus II of USP Sao Carlos satellite view and resulting map shown in red. . . 58

6.10 Results of obstacle detection method in a range image. White pixels are obstacles and black pixels otherwise. . . . . . . . . . . . . . . . . . . 59

6.11 Vizualization of VirtualScan in red over all white points from 3D-LIDAR Velodyne HDL-32E. Note that white points are in 3D coordinates while red points are in $2 \mathrm{D}$ coordinates. . . . . . . . . . . . . . . . . . 60

6.12 Pictures of public demonstration of CaRINA-2 . . . . . . . . . . . 61

7.1 Example of future work: Detection and tracking of moving obstacles. . . . . . 64

7.2 2D map created with just a single frame of gray-scale image and 3D points generated by a 3D-LIDAR. . . . . . . . . . . . . . 66 


\section{List of Tables}

5.1 Chosen parameters in this experiment. . . . . . . . . . . . . . 31

5.2 Rank of road area estimation in online Kitti Benchmark (December,2014). MaxF is the maximum $\mathrm{f} 1$-measure. . . . . . . . . . . . . . . . . 32

5.3 Road-area evaluation [\%] in a Testing Dataset. Online available . . . . . . . . 33

5.4 Comparison of several precision-degree in road-estimation evaluation [\%] using the ground-truth images as estimated-obstacles. The results shows that is not possible to achieve $100 \%$ of MaxF due to only ego-road be detected. . . . . . . 38

5.5 Road-area evaluation [\%] of (GRES3D+VELO) in a Training Dataset. . . . . . 39

5.6 Road-area evaluation [\%] of (GRES3D+SELAS) in a Training Dataset. . . . . 43

5.7 Road-area evaluation [\%] of (GRES3D+SGBM) in a Training Dataset. . . . . . 46 


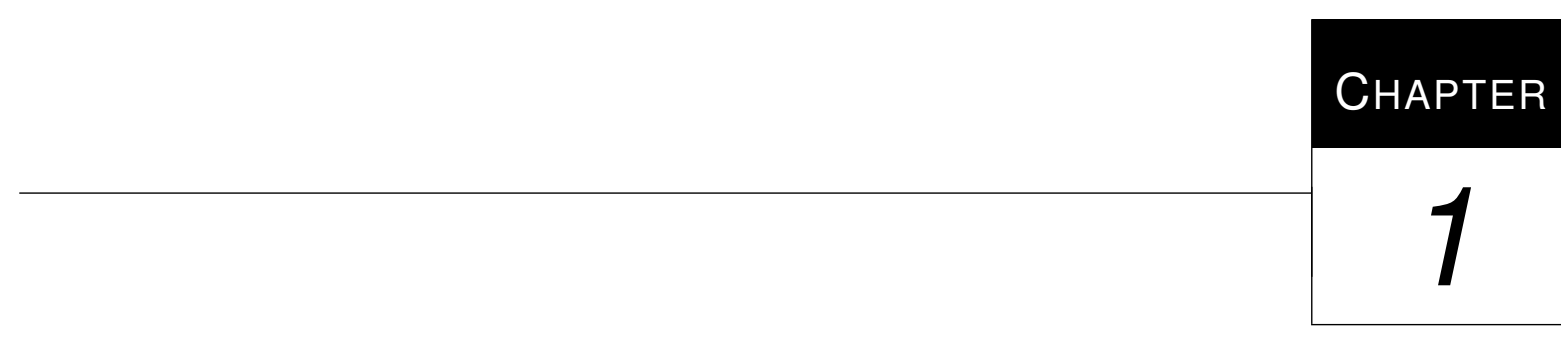

Introduction

"Thus, the task is not so much to see what no one yet has seen, but to think what nobody yet has thought about that which everybody sees."

(Arthur Schopenhauer)

According to world health organization (WHO, 2014), each year, approximately 1.2 million lives are lost due to traffic accidents worldwide, and around 50 million people suffer non-fatal car accidents. The majority of these are due to human error, like small distractions when drivers use a mobile phone or eat something, or more seriously faults like drunk driving or exceeding the speed limit. Advanced Driver Assistance Systems (ADAS) and Self-driving cars promise to reduce this number of deaths and injuries. In addition, this technology can bring other benefits like optimal fuel usage, efficiency with freight transportation and traffic flow improvement in big cities, providing transportation for children who are not allowed to drive, and adults unable to drive such as physically handicapped, visually impaired or elderly people.

Recent progress in self-driving cars research shows the viability of this technology for the next years (Google, 2014; Ziegler et al., 2014). While vehicle control and trajectory planning algorithms have already been demonstrated successfully, the environment perception task is still an unsolved problem. Among the various perception tasks, the obstacle detection and terrain 
classification are two of most fundamental abilities that a mobile robot requires to act over an environment. In the Intelligent Vehicles context, commercial ADAS like Adaptive Cruise Control (ACC) and Forward Collision Warning (FCW) depend on obstacle detection output to detect and notify the driver about a risky situation. Nevertheless, such systems still requires use of expensive sensors or are limited to specific conditions as flat surface or geometric appearance. In some cases, they can detect only obstacles of a minimum height or size. Several terrain classification systems used for intelligent vehicles are also known as road detection or lane detection systems being the last one an input to commercial ADAS called Lane Departure Warning (LDW) and Lane Keeping System (LKS). Unfortunately, these systems are dependent on well maintained road markings, or good light conditions to detect a pattern of colors, textures or intensities from a specific sensor. In most cases, statistical techniques associated to temporal information are used to treat possible errors caused by sensor noise, and limitations of the detection model. The most common type of approach used to detect the road-model is based on machine learning methods, which can perform with impressive results but is very dependent of a good training-database.

Since it is an expensive and complex job to create and maintain huge databases of road scenarios of several and very different scenarios (e.g. Brazil, Germany, South Africa and India), adaptive and/or self-supervised methods are good candidates for robust detection systems for the future. Due to that, this thesis presents a method to estimate obstacles and estimate the road terrain avoiding the most common assumptions used by works presented in literature over the past years. It is a method to help machine learning techniques as a preprocessing step, therefore the fundamental requirement it is the low runtime to be computed in parallel with a classification system. Since stereo image sensors have low-cost sensors, automotive industries (Bosch, 2014; Daimler, 2014) have shown interest in using then in intelligent vehicle applications. Due to that, the present work focus on this type of sensor and get comparable performance and runtime with costly sensors approaches (3D-LIDAR). In particular, this thesis is set out to answer the following questions:

- How to detect the road area with limited prior knowledge (training) and avoiding strong assumptions like specific shape, or flat surface?

- Can we use stereo image sensor as an alternative to 3D-LIDAR sensor to detect the road area in regular urban scenarios?

- Since the density of a disparity map from correspondence algorithms directly influences the runtime, can we detect obstacles/road using a non-fully dense disparity map? 
In order to evaluate and compare our approach with different sensor systems (stereo image and 3D-LIDAR sensors), this thesis make use of an experimental data from AnnieWay platform that is online available on KITTI Benchmark ${ }^{1}$. More specifically, the KITTI-ROAD ${ }^{2}$ dataset have been used in the experimental evaluation of this work.

This thesis is also part of a larger project called CaRINA (Carro Robótico Inteligente para Navegação Autônoma ${ }^{3}$ ) developed in the Mobile Robotics Laboratory (LRM, 2014) with support granted by (CAPES, 2014; CNPq, 2014; FAPESP, 2014). CaRINA project aims to research and develop technologies of ADAS and autonomous navigation systems to urban environments. It began in April 2010 with an electric service car, which has been modified for computer control and instrumented with a variety of sensors for perception. In October of the same year the vehicle (known as CaRINA-1) was performing its first autonomous control test. Few months later CaRINA-1 was able to autonomously traverse a $1 \mathrm{~km}$ trajectory in the university campus. The work on CaRINA-2 (a standard Fiat Palio Adventure) started in July 2011. One year later, to the best of our knowledge it has been the first commercial vehicle from Latin America capable to perform autonomous navigation in public streets ${ }^{4}$. Figure 1.1 shows CaRINA 1 and 2 research platforms.

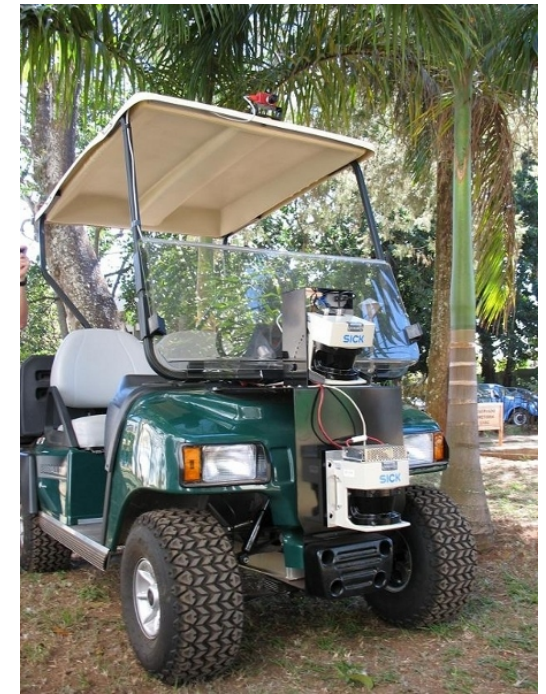

(a) CaRINA-1

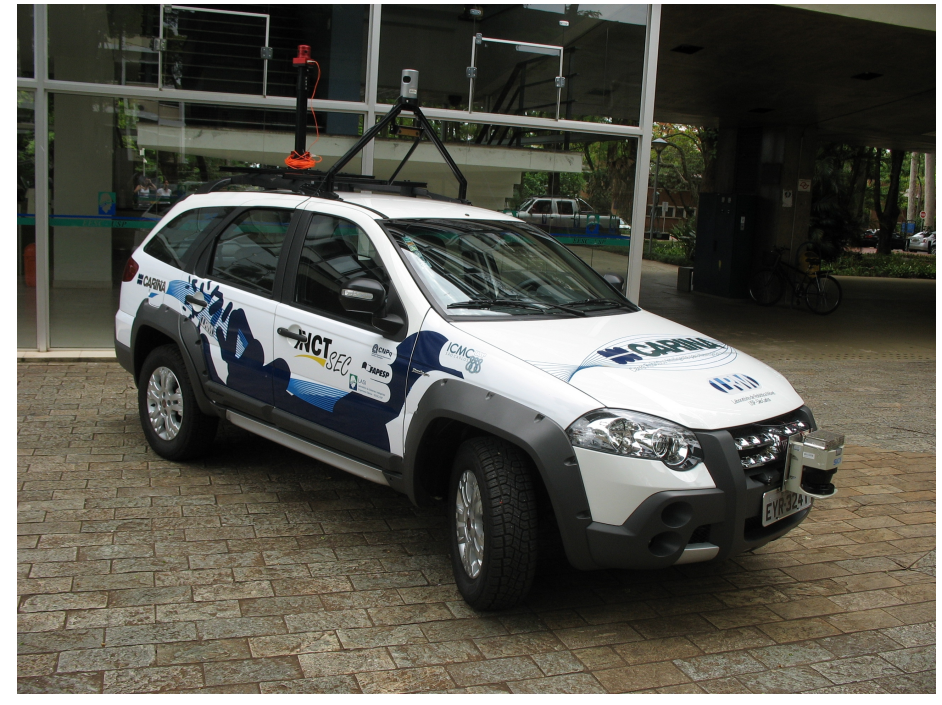

(b) CaRINA-2

Figure 1.1: The vehicular robotic platforms of CaRINA project.

\footnotetext{
${ }^{1}$ http://www.cvlibs.net/datasets/kitti/

${ }^{2}$ http: //www.cvlibs.net/datasets/kitti/eval_road.php

${ }^{3}$ In English means Intelligent Robotic Car to Autonomous Navigation

${ }^{4}$ http: / / www . youtube.com/ Irmicmc
} 


\subsection{Problem Statement and Objectives}

Given a sensor system installed in a vehicle platform capable of generate a single frame of data in a 3D point cloud format, and a screen projection function (provided by a calibration methods), this work tries to detect the road terrain in the screen plane (see Figure 1.2) based on few and weak assumptions. For instance and without loss of generality, the screen plane can be interpreted as an image provided by a camera. The major assumptions used in this work is that the vehicle platform is already on the road, the vehicle platform is not upside down, and the road is delimited by curbs and obstacles. This is reasonable for urban scenarios.

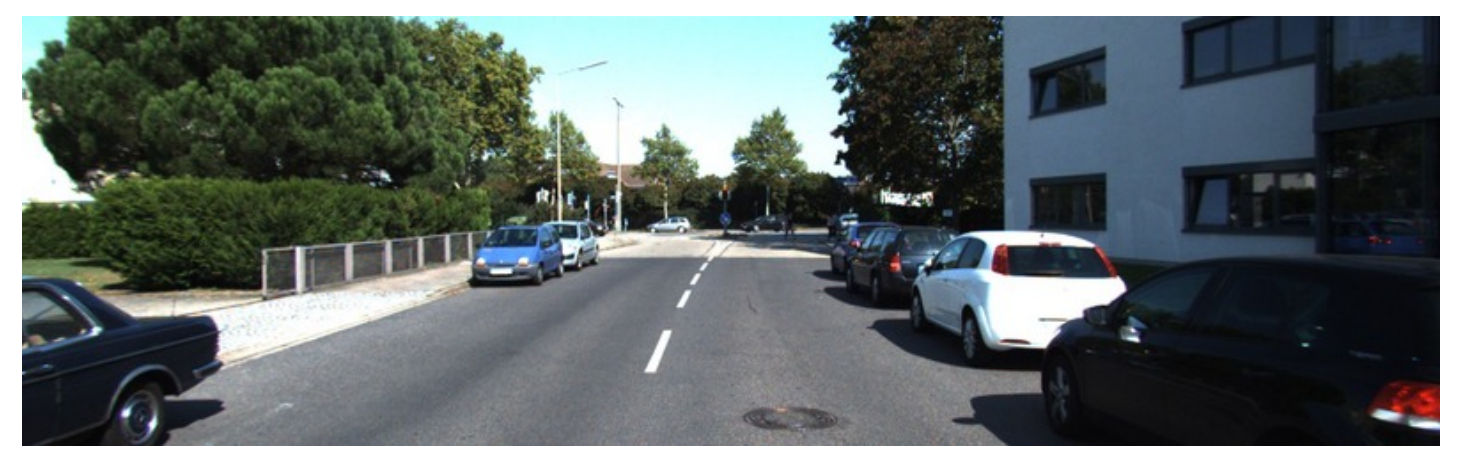

(a) Raw color image that represents an arbitrary screen plane.

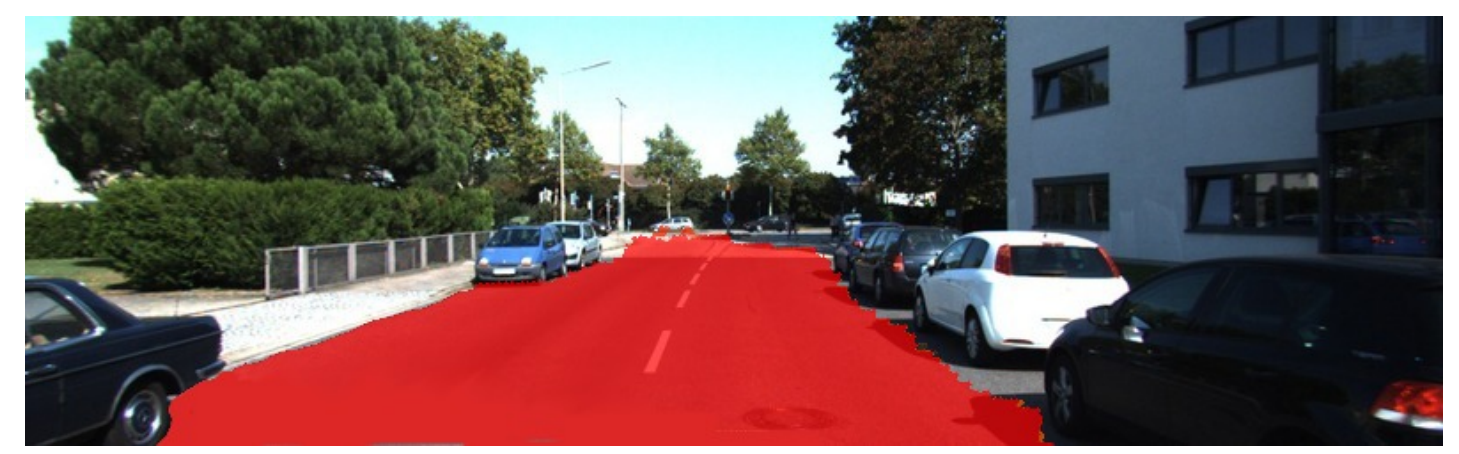

(b) The expected road area to be detected by present method.

Figure 1.2: Given a single 3D point cloud read from a sensors system installed in a vehicle platform and a screen projection function, the present work tries to detect the road terrain in the screen plane. (Images from KITTI-ROAD Benchmark dataset).

Since this work aims to explore and evaluate a new approach for an intelligent vehicles application, the processing time is take into account in a standard hardware configuration, i.e. no General Purpose Graphical Processor Unit (GPGPU) and no Field-Programmable Gate Array (FPGA). Also, the proposed model is free of training or previous map information. It does not use temporal information, i.e. our model does not accumulate information between frames. 
The entire work is divided in two major parts, the first one to detect obstacles and the second one to estimate the road area. Our obstacle detection method uses sparse 3D points projected in a perspective view to generate a graph based on screen coordinates. After that, it calculates an obstacle-confidence degree for each node using a spatial-condition represented by all edges of the graph. The second part is a fast road estimation method that uses obstacle-confidence as input to estimate several confidence maps of road area in an screen plane and combine them.

\subsection{Thesis Contributions}

The contributions of this thesis are as follows:

- A data structure of 3D point clouds that combined with perspective view creates a neighbourhood information for each point allowing to estimate obstacles using only a local spatial-condition, i.e., no constants like minimum-height.

- A distribution of 3D points over the screen plane that allow to estimate the road area without training methods or assumptions about the geometry features and shape. This model can be applied in different sensors systems.

- A model capable to perform estimation of obstacles and road area avoiding the requirement of process all pixels from a pair of images (provided by stereo cameras). This model allow us to perform modified versions (sparse version) of stereo matching algorithm to improve runtime.

- An approach to use stereo cameras as an alternative to 3D-LIDAR in day time conditions without hardware improvements (no FPGA or GPGPU).

- A fast method that estimates obstacles in a stereo image with low runtime using only local information. One of its advantages is that it is free of assumptions about size, height or shape of common urban objects, therefore cars, pedestrians, posts and most importantly, curbstones can be detected with the same algorithm. The height of the lowest detected obstacle is determined by the accuracy of $3 \mathrm{D}$ point cloud and by spatial-condition's parameters.

- A fast road estimation method for screen planes is presented. This method does not dependent on road markings, texture-patterns, shape or geometric features as flat surface and height. 


\subsection{Applications}

In this section we highlight some applications that can take advantages of the contributions of this work:

- ADAS: While the proposed Obstacle Estimation method can be used by several ADAS like ACC, FCW and Park Assistance. The Road Estimation can be used by road following systems in unmarked roads, and by a pre-processing method before detect road markings.

- Object Classification and Tracking: The proposed Obstacle Estimation method can be used as input in Vehicle/Pedestrian Detection method for a posterior classification and tracking.

- Autonomous Navigation Systems: The road/obstacle estimation methods can be used to generates inputs to an reactive autonomous navigation systems.

- Automatic Mapping of Road Network: One of current issues for autonomous vehicles is the ability of a easy creation and maintaining of precise and updated maps of urban environments. Since a city is continuously expanding and changing, the road network also changes the number and position of traffic signs, lanes, and intersections. Therefore, since the proposed Road Estimation method is free of several assumptions, it can be used to compose an automatic mapping system of road network.

\subsection{Thesis Outline}

This thesis is structured as follows: Chapter 2 shows a brief discussion of related works in autonomous vehicles, obstacle detection and road detection. Chapter 3 presents the proposed obstacle estimation method with two different sensor configurations. Chapter 4 presents the proposed road estimation method with some techniques to improve it and detect some semantic information. Chapter 5 describes experimental evaluations of road detection in some different perspectives. Chapter 6 describes other contributions derived from this thesis. Finally, the conclusions are presented in Chapter 7. Published papers and other academic works published while the production of this thesis are shown in the Appendix section. 


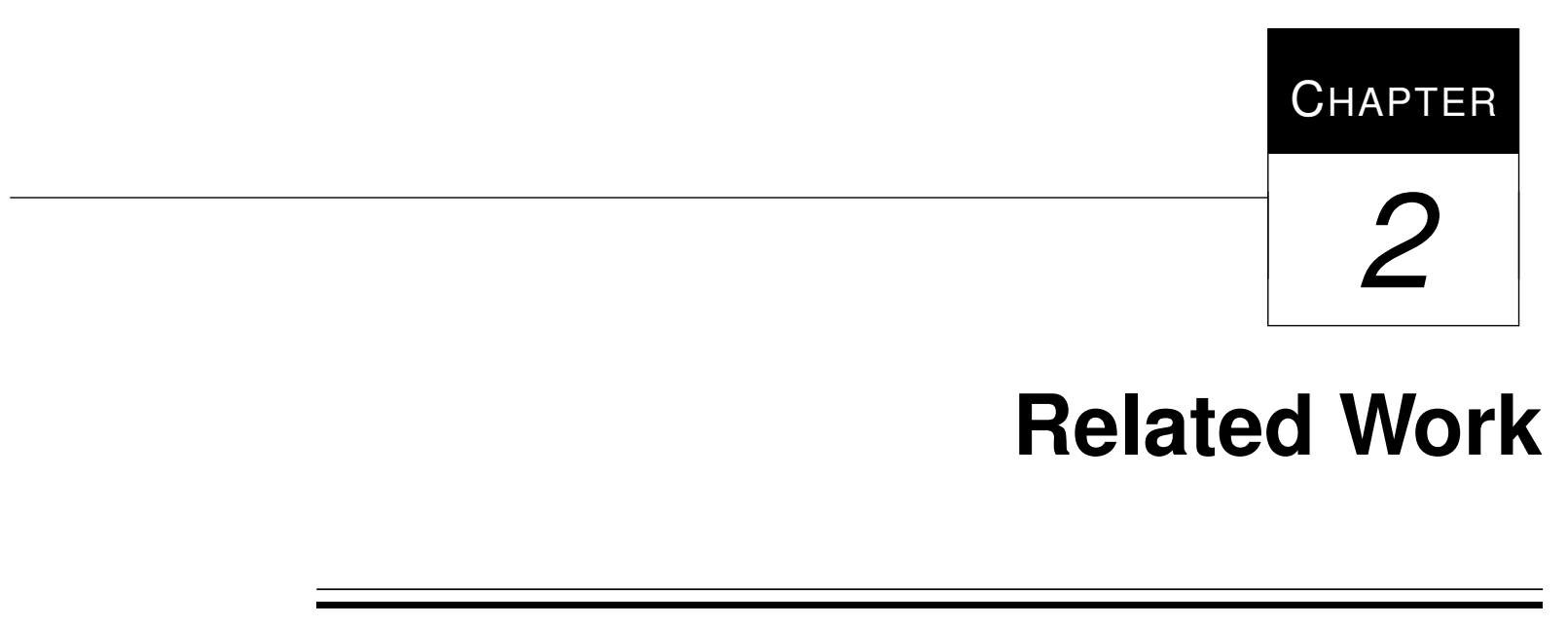

In the last 10 years, the Defense Advanced Research Projects Agency (DARPA) encouraged the autonomous vehicles research area by the organization of three big competitions. The Grand Challenges (Buehler et al., 2007), performed in 2004 and 2005, had focus on crossing the desert and skills known as "road following" and "obstacle avoidance". The first edition was disappointing, the best team traveled just $12 \mathrm{Km}$ from $224 \mathrm{Km}$ proposed by DARPA. In 2005 , this challenge was proposed again and five vehicles finished the new route. In 2007, perhaps the most famous and relevant competition was launched, the Urban Grand Challenge (Buehler et $a l ., 2009$ ) where robots-cars should be able to safely navigate with respect to other robots-cars, human-driven vehicles, and the urban environment. They had also to obey some of the traffic rules from California. This competition had 11 finalists teams and 6 robots finished the route of $96 \mathrm{Km}$ completely. Despite the impressive results of the 2007 edition which proved the feasibility of this kind of technology, it is important to highlight that the autonomous navigation in urban environments problem was simplified in order to evaluate the robots and then perform this competition.

In Europe, the European Land-Robot Trial (ELROB) (ELROB, 2012) is a initiative similar to DARPA competitions but without a winner. It is performed every year since 2006 alternating between military and civilian approaches. Unlike ELROB, Europe has initiatives where companies, universities and research centres have cooperation projects to develop technologies to improve safety and intelligence of the vehicles. We can mention "The European 
Field Operational Test" (EuroFot) (EuroFOT, 2012) and "Highly Automated Vehicles for Intelligent Transport" (HAVEit) (HAVEit, 2012) with focus in the development of systems like ACC (Adaptative Cruise Control), FCW (Foward Collision Warning), BSIS (Blind Spot Information System), LDW (Lane Departure Warning) and other. Finally, in 2011, Europe hosted the "Grand Cooperative Driving Challenge" (GCDC) (GCDC, 2012) that boosted research in systems of cooperative-driving, where a robot can talk with other robots and infrastructure in order to negotiate its planning and control. This challenge will repeat in 2016 as a second edition of a challenge in cooperative-driving systems.

Despite the high importance that the latest initiatives and competitions received, it is important to highlight that the development of autonomous vehicles has been studied since 80 s by various research groups around the world, such as the ARGO project (Broggi et al., 1999), VaMoRs vamp (Gregor et al., 2000), NavLab (Thorpe et al., 1988), and several others. Even today there are research projects on autonomous vehicles with different approaches and prototypes such as AUTONOMOSlabs (AUTONOMOSlabs, 2012) that has two vehicles - the "Spirit of Berlin" and "Made In Germany" which in 2012 conducted the "Mission: Brandenburger Tour" where the vehicle travelled on the streets and highways of Berlin without driver's actuation. Another group which also has projects with smart cars is VisLab (VISLAB, 2012) that created several prototypes - and some of most recent projects are "BRAiVE" (2009), "VDCL Grandeur" and "Porter" (2010) that travelled a route of 13,000 km from Italy to China.

Although several research groups have been actively working on autonomous vehicles around the world, few initiatives take place in Latin America. One of the first projects in this area is developed at the Federal University of Minas Gerais. Its software architecture is described in (V.B. Sabbagh, 2010). Experiments of autonomous navigation are reported in (D.A. Lima, 2010; de Lima e Pereira, 2013). Researchers from Federal University of Itajuba has also been working on autonomous navigation (L. Mg, 2010) and parking (L.L.G. Veermas, 2010). Both groups have fully automated cars and have been testing navigation algorithms in open spaces. A commercially automated vehicle has been recently acquired by Federal University of Espirito Santo, which has been actively working on obstacle detection using stereo vision (L. Veronese, 2011). Some research groups have also been working on algorithms such as: pedestrian recognition (H. Roncancio, 2012), autonomous parking (M. Heinen, 2006), and collision risk estimation (A. Miranda Neto, 2013). Besides commercial vehicles, other types of outdoor robotic platforms have been also used to autonomous navigation (S.S. Bueno, 2009).

Currently, perhaps the most famous vehicle and considered one of the state of the art is the "Google Self-Driving Car" (Google, 2012) that took as its starting point, all technology developed in the DARPA Urban Challenge. In the industry community, several companies like Volvo and Mercedes already presented, in 2014 and 2015, their concept products (Mercedes, 
2015; VOLVO, 2015). Unlike Google, the main characteristic of these new prototypes is the absence of high-cost sensors. More specifically, these vehicles are using the fusion of radars and cameras instead 3D-LIDAR sensor. Thus, the industrial community has encouraged the researchers to develop solution for road detection and obstacle detection using low cost sensors (radar and camera).

Among the topics addressed in this thesis, tasks such as obstacle detection and detection of road has been researched by computer vision and robotics community by many years. Visual road detection, also known as "lane detection" and "road following", has been one of the desirable skills to improve autonomous vehicles systems. As a result, visual road detection systems have been developed by many research groups, such as (Broggi et al., 1999) (Gregor et al., 2000) (Thorpe et al., 1988). Details about these and other works can be found in several surveys (Bonin-Font et al., 2008) (Desouza e Kak, 2002) (Bertozzi et al., 2000) (Dickmanns, 1998), (Bar Hillel et al., 2012). Most often, these algorithms aim at finding edges as road delimiters, try to match geometric models (Crisman e Thorpe, 1991), detect lane-markings (Takahashi et al., 1999) (Bertozzi e Broggi, 1998) or detect vanishing points (Kong et al., 2009) to detect or estimate the drivable space ahead of the vehicle (Enzweiler et al., 2013), (He et al., 2004; Jung e Kelber, 2005). These approaches are often limited to paved, structured and well-maintained roads as they make use of some image processing techniques that require threshold parameters. Some works based on machine learning methods obtain texture and color in a previous training step, where a human annotates the road area manually (Kong et al., 2010; Kuehnl et al., 2012; Shinzato et al., 2012). These vision approaches with supervised machine learning fail if they are evaluated in scenarios that differ from those in the training data, like system trained to asphalt but used in gravel road surfaces or cobblestone surfaces of old cities. In adaptive approaches, the system assumes parts of image that will always have the same label (Tan et al., 2006) which can easily fails due the large difference between urban scenarios across the world.

For approaches that use LIDARs, a planar road assumption was required in (Moosmann e Stiller, 2013) and a local convexity feature was introduced in (Moosmann et al., 2009) to distinguish road surface from objects in LIDAR data. Road estimation can also be based on an intensity measurement processing to detect lane-markings (Kammel e Pitzer, 2008). Furthermore, the estimation of roughness for segmentation and detection of road delimiters was proposed by (Häselich et al., 2011).

In some cases, the road detection is a modification of an obstacle detection system to detect small obstacles like curbs and then estimates the road area delimited by them. These approaches are known as "curb detection systems", and can use several types of sensors or combination of them. In a simple approach, (Shin et al., 2010) extracts the curbs by the 1D laser scanner while (Oniga et al., 2008) uses a stereo image sensor and the Canny algorithm to find the curbs 
directly. (Siegemund et al., 2010) find the curbs by using a stereo image sensor with conditional random field (CRF) to propagate the curb detection result. (Aufrere et al., 2003) detect the curbs using a 1D laser scanner and a camera together. (Zhao e Yuan, 2012) uses the 3D-LIDAR (64 rings) to detect curbs and obstacles based on three spatial cues, elevation difference, gradient value and normal orientation. Such approaches are based on data structures, as grid and voxels and are limited due to the need of parameter definitions, like intensity threshold values, cell size, and minimum height difference. Grid approaches do not work properly for distant objects, since few points hit the same cell and lose accuracy, depending on the cell size. In order to avoid such problems, many approaches accumulate few scans (temporal information) before performing the classification or make use of estimation methods to improve the detection model.

Most common approaches require several assumptions or conditions to work appropriately. One common requirement of visual approaches is the need of previous tuning of parameters or training of model with guarantee that the training dataset has samples of all types of scenario. The LIDAR approaches have requirements like minimum height and does not work well in far ranges. In this thesis, we have developed an obstacle detection and road estimation method that requires a very limited number of parameters without requirements of temporal information (accumulation of frames) or a priori knowledge of the space (map). 
This chapter presents an obstacle estimation method that makes use of sparse 3D points projected in a screen plane. The main idea is to calculate a confidence degree for each point based on a spatial-condition between it and its neighbours. This confidence degree represents how likely a point is part of an obstacle (details in section 3.1). However, before calculating this confidence, the system needs to pre-process a raw sensor data in a structure that also describes the neighbourhood between all points (section 3.2). This structure is a graph and must be generated by a preprocessing step that is dependant of sensor systems used (section 3.3 and section 3.4).

\subsection{Definition of Obstacle by 3D Spatial-Condition}

Our obstacle detection method is an evolution of works already presented on literature by (Matthies et al., 1995, 1998; Talukder et al., 2002) in which the obstacle detector should work in unstructured environments as cross-country terrain. Their technique measures slope and height of surface patches, where a "slope" is measured by the angle formed by the surface and the vertical axis. Figure 3.1 shows an example of a 1D-range profile, where slant $(\theta)$ and height $(H)$ are shown for two different surface patches. The intuition behind this approach is: if a surface patch has limited slope then it belongs to ground surface, but if this patch is steep, it corresponds to an obstacle large enough to be considered by the robot. Thus, the lower patch 
in Figure 3.1 would probably be considered traversable, while the higher patch would probably be considered an obstacle.

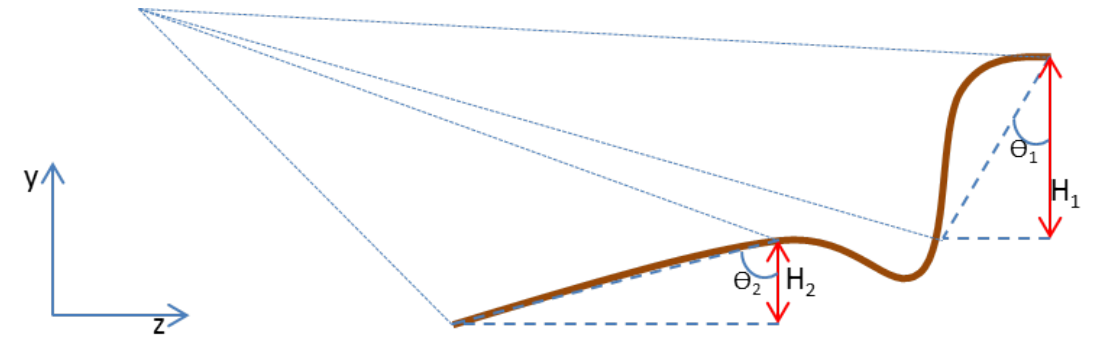

Figure 3.1: Intuition behind the algorithm from (Matthies et al., 1995) and (Talukder et al., 2002). The blue lines represents the laser projection of a lidar sensor. The higher patch with $H_{1}$ would probably be considered obstacle, while the lower patch with $\mathrm{H}_{2}$ would be considered traversable. Image adapted from (Talukder et al., 2002).

The technique of (Matthies et al., 1995) looks exclusively at 1D range profiles as shown in Figure 3.1, which makes the algorithm very fast, but has drawbacks in terms of detection accuracy. In (Talukder et al., 2002), they present an improved version of obstacle detection which computes 3D slopes. The "obstacles" are defined in terms of two distinct points in 3D space, i.e., given two 3D points $p_{1}=\left(p_{1 x}, p_{1 y}, p_{1 z}\right)$ and $p_{2}=\left(p_{2 x}, p_{2 y}, p_{2 z}\right)$, these points are considered to be obstacles if they satisfy two conditions:

1. $H_{r}<\left|p_{2 y}-p_{1 y}\right|<H_{\max }$ i.e. their difference in height (along $y$ axis) is larger than $H_{r}$ but smaller than $H_{\max }$.

2. $\frac{\left|p_{2 y}-p_{1 y}\right|}{\left|p_{2}-p_{1}\right|}>\cos \left(\theta_{r}\right) \quad$ i.e., the line joining them forms an angle with the horizontal plane larger than $\theta_{r}$.

where $H_{r}, H_{\max }$ and $\theta$ are suitably chosen constants.

Both approaches were developed to work with range images or 3D-reconstruction from disparity maps, i.e., (Matthies et al., 1995) and (Talukder et al., 2002) methods works with images where each pixel represents a point in, respectively, 1D and 3D space. Our obstacle detection method is an evolution of these approaches since it is not restricted to structured data as images, the constants of height are not required any more and instead a binary output, our method computes a continuous value that represents a confidence degree about $p_{1}$ be classified as an obstacle. The confidence degree is computed by a function that remap the condition 2 from (Talukder et al., 2002) to a sigmoid-like function defined as in Equation (3.1):

$$
\operatorname{sigmoid}\left(\frac{\left|p_{1 y}-p_{2 y}\right|}{\| p_{1}-p_{2}||}, \cos (\theta), \sigma\right)
$$


where $\left|p_{1 y}-p_{2 y}\right|$ corresponds to the absolute difference of height values between these two points, $\left\|p_{1}-p_{2}\right\|$ is the $3 \mathrm{D}$ euclidean distance between points $p_{1}$ and $p_{2}, \theta$ and $\sigma$ are suitably chosen constants, and $\operatorname{sigmoid}(x, \mu, \sigma)$ is a function defined as in Equation 3.2:

$$
\operatorname{sigmoid}(x, \mu, \sigma)=0.5+\frac{0.5(x-\mu) \sigma}{\sqrt{1+(x-\mu)^{2} \sigma^{2}}},
$$

where $\mu$ specifies the effective threshold and $\sigma$ is a scale parameter that influences the tangent slope at the threshold (Fig. 3.2).

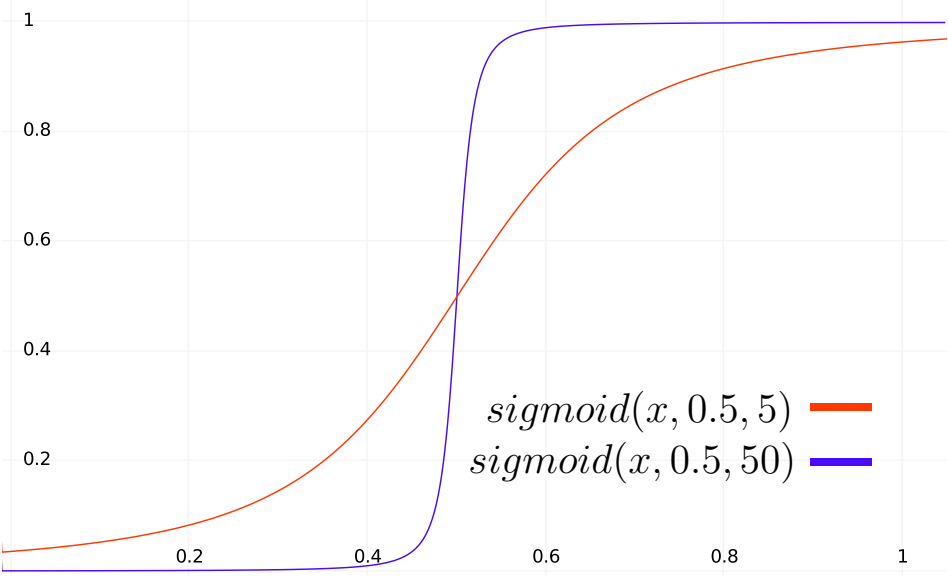

Figure 3.2: The sigmoid-like threshold function is used to map an arbitrary value calculated between a pair of points for the [0,1] interval depending on parameters $\mu$ and $\sigma$.

In order to treat sensor noise, it is important that the estimation value of a point $p_{1}$ does not depend on only one neighbour, and instead, the algorithm should compute this value using all neighbourhood of $p_{1}$. To do that, our method requires a data structure that describes the neighbourhood between all points inside the screen plane. Therefore, this thesis also proposes a general model of 3D point clouds where the neighbourhood of each point is defined by the distance between screen coordinates in 2D plane of each point. This model results in a graph where the edges are created by a planar triangulation method.

\subsection{General Model of 3D Point Clouds in Screen Plane}

Given a set of points of type $p_{a}=\left\{x_{a}, y_{a}, z_{a}\right\}$ relative to an arbitrary coordinate system, and a function $T()$ that calculates screen coordinates $(u, v)$ of $p_{a}$, a preprocessing step can create a undirected graph $G=\{P, E\}$. Where $P$ is the set of nodes that represents sparse 3D points and $E$ is the set of all edges that guarantee that there exists no other point between two points 
connected by an edge (see Figure 3.3). Every node, in our approach, must have 3D coordinates $(x, y, z)$ and screen coordinates $(u, v)$. The $3 \mathrm{D}$ coordinates are used only to estimate obstacle points and screen coordinates are used for the generation of all edges.
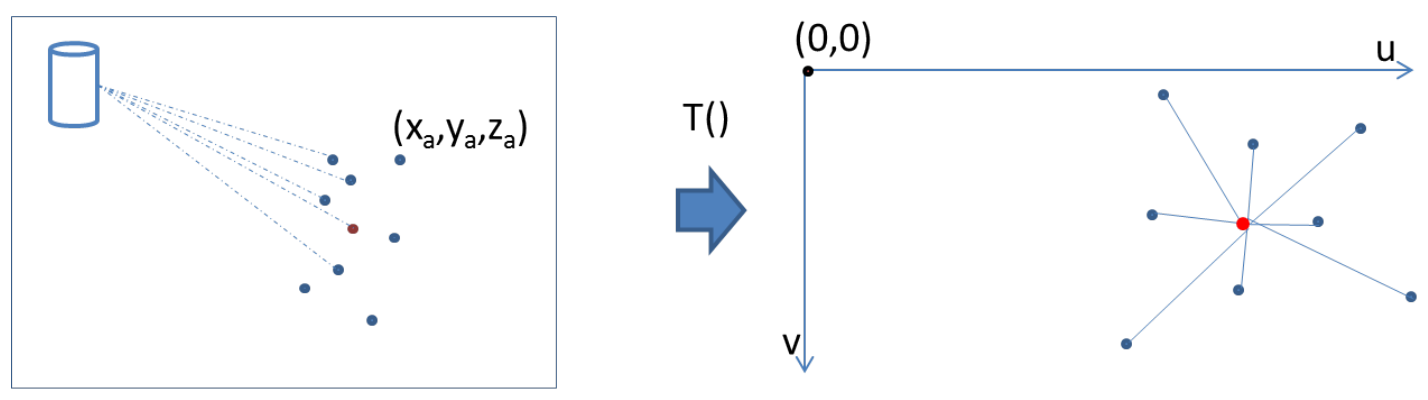

Figure 3.3: Generic Modelling of 3D point clouds in screen plane. The cylinder of left image represents a 3D-lidar in the 3D world, in this work also represents the origin point $(x=0, y=0, z=0)$. The right image shows a screen plane (image world) with all points from 3D-lidar projected inside it.

To generate the set of edges $E$, the screen coordinates $(u, v)$ and the planar Delaunay Triangulation (Lee e Schachter, 1980) are used. This triangulation method copes with situations in which the present model cannot use a structured neighbourhood like 4-connected or 8-connected pixels in kernel-filters from image processing methods. These situations happens when the distribution of points is not regular, which is very common in stereo camera approaches when the disparity map is not fully dense (Figure 3.4a), or in projection of sparse points from 3D-LIDARS (Figure 3.4b) in an image. The Delaunay Triangulation properties also guarantee that there is no other point between the points from Delaunay's edges. All edges created in this step represent the local-relationship between two points and are used by the obstacle estimation function.

In this work, the present model is implemented and described with two different sensor systems to a comparative evaluation: the first acquires 3D points $(x, y, z)$ from high-precision 3D-LIDAR (Velodyne HDL-64E ${ }^{1}$ ) and with intrinsic and extrinsic parameters computes screen coordinates $(u, v)$ into monocular camera. The second computes disparity maps from a stereo camera and uses screen coordinates to compose the triple \{column, line, disparity $\}$ as 3D points $(u, v, d)$. Is important to notice that both approaches require a previous calibration step, where intrinsic and extrinsic matrices are computed. Since we used an online dataset, these matrices are already provided and are computed by an automatic calibration method described in (Geiger et al., 2012b) that is also available online to extract matrices from other sensors system.

\footnotetext{
${ }^{1}$ Velodyne HDL-64E is a LIDAR sensor that integrates several lasers into one rotating housing.
} 


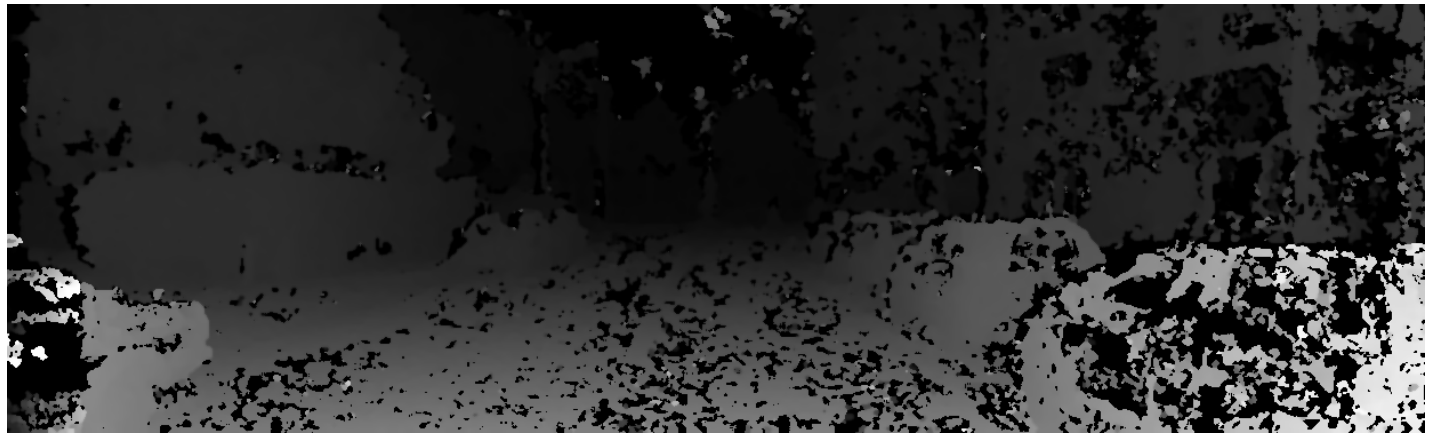

(a) Black pixels does not have valid disparity value, so can not generate a node.

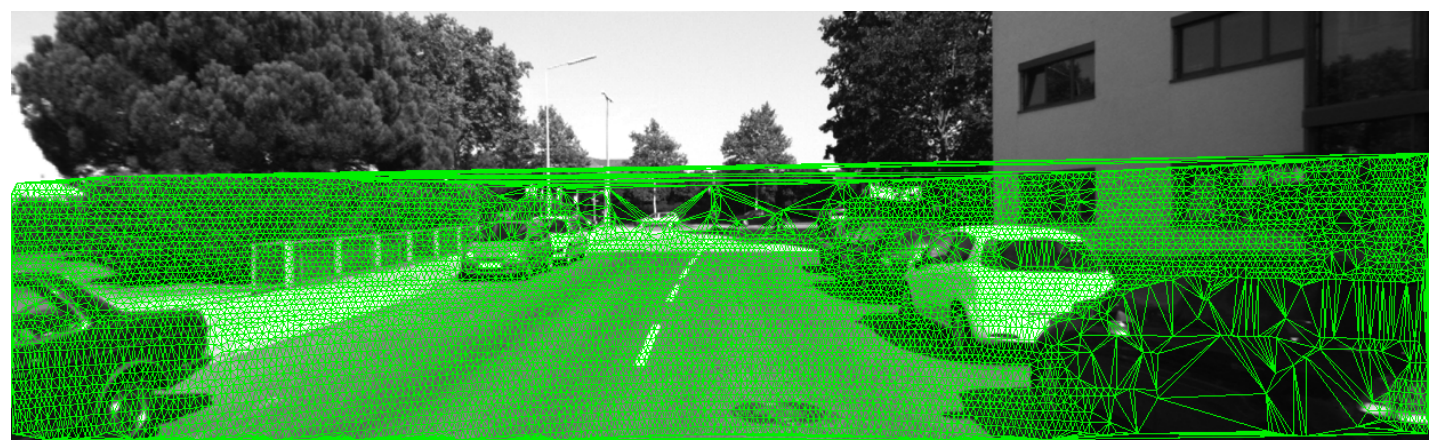

(b) Edges between 3D-LIDAR points. These points sometimes, have big distances between them as in right part of this sample.

Figure 3.4: Situations where the model cannot use structured neighbourhood.

\subsection{D LIDAR + Monocular Image Sensor}

The sensor type known as LIDAR, from Light Detection And Ranging, works emitting a beam of electromagnetic radiation to a specific direction. For each beam, the sensor uses the delay time until it returns to calculate the distance between the emitter and a surface. This distance joined with some calibration parameters creates a point in the specified dimension space that the sensor was calibrated for. Therefore, a 3D-LIDAR sensor give us a large amount of 3D points called 3D point cloud. The Veloyne HDL-64E sensor (used in this work) generates a $3 \mathrm{D}$ point cloud at $10 \mathrm{Hertz}$ in a range of 360 degree and 80 meters of maximum distance. For this sensors system, the present model projects a sparse and unstructured 3D point cloud into an image. As described in (Geiger et al., 2013), we used intrinsic and extrinsic parameters to transform a 3D point $p_{l}=\left(x_{l}, y_{l}, z_{l}, 1\right)^{T}$ relative to 3D-LIDAR coordinate frame into a 3D point $p_{c}=\left(x_{c}, y_{c}, z_{c}, 1\right)^{T}$ in a camera coordinate frame using Equation 3.3:

$$
p_{c}=\left(\begin{array}{cc}
\mathbf{R}_{\text {rect }}^{0} & 0 \\
0 & 1
\end{array}\right)\left(\begin{array}{cc}
\mathbf{R}_{\text {velo }}^{\text {cam }} & \mathbf{t}_{\text {velo }}^{\text {cam }} \\
0 & 1
\end{array}\right) p_{l}
$$


where $\mathbf{R}_{\text {rect }}^{0}$ is the rotation matrix $(3 \times 3)$ from a raw-image-camera to a rectified-image-camera, $\mathbf{R}_{\text {velo }}^{\text {cam }}$ and $\mathbf{t}_{\text {velo }}^{\text {cam }}$ are, respectively, the rotation matrix $(3 \times 3)$ and translation vector $(3 \times 1)$ that transform a point from a 3D-LIDAR coordinate frame into a camera coordinate frame.

The projection matrix $\mathbf{P}_{\text {rect }}^{(i)}$ from the $i^{\text {th }}$ image-plane is applied to all 3D points $p_{c}$, as shown in Equation 3.4, for the obtaining of the screen coordinates $(u, v)$.

$$
\left(\begin{array}{l}
u \\
v \\
1
\end{array}\right)=\left(\begin{array}{c}
x / w \\
y / w \\
w / w
\end{array}\right)=\left(\begin{array}{cccc}
f_{u}^{(i)} & 0 & c_{u}^{(i)} & -f_{u}^{(i)} b_{x}^{(i)} \\
0 & f_{v}^{(i)} & c_{v}^{(i)} & 0 \\
0 & 0 & 1 & 0
\end{array}\right)\left(\begin{array}{c}
x_{c} \\
y_{c} \\
z_{c} \\
1
\end{array}\right)
$$

After applying Equation 3.4 (see (Geiger et al., 2013) for details), points that are not inside the image or have value $x_{c}<0$ are removed. The remaining points compose set $P$ of nodes $p=\left(x_{c}, y_{c}, z_{c}, u, v\right)^{T}$ that can be projected in an image or can be viewed in a 3D space (see Figure 3.5).

\subsection{Stereo Image Sensor}

According to Forsyth e Ponce (2002), a general definition to stereo vision is a method composed by two processes: The fusion of features observed by two eyes and the 3D reconstruction of image. In practice, Bradski e Kaehler (2008) defines that the stereo vision is composed by four steps: Mathematically $(i)$ removal of radial and tangential lens distortions (undistortion), (ii)rectification process, $($ iii) find the same features in left and right camera views known as correspondence step, and finally $(i v)$ transform the disparity map into distances by triangulation in the reprojection step (see example in Figure 3.6). For details about these four steps, the reader is referred to (Bradski e Kaehler, 2008).

In this work, our obstacle estimation method requires the computation of only the first three steps of (Bradski e Kaehler, 2008) definition, where the correspondence step is computed by the Efficient Large-Scale Stereo Matching (ELAS) (Geiger et al., 2010). ELAS is a stereo matching algorithm that is capable of computing disparity maps at large-scale image resolutions with very low runtime. The method can handle stereo correspondences that are highly ambiguous, assuming piecewise smooth disparities to create support points that compose a $2 \mathrm{D}$ mesh and carry valuable prior information for the remaining disparities. The algorithm automatically determines the disparity range, can be easily parallelized and has shown impressive performance on the realistic KITTI dataset (Geiger et al., 2012a) and on the large-scale Middlebury benchmark (Scharstein e Szeliski, 2002). 


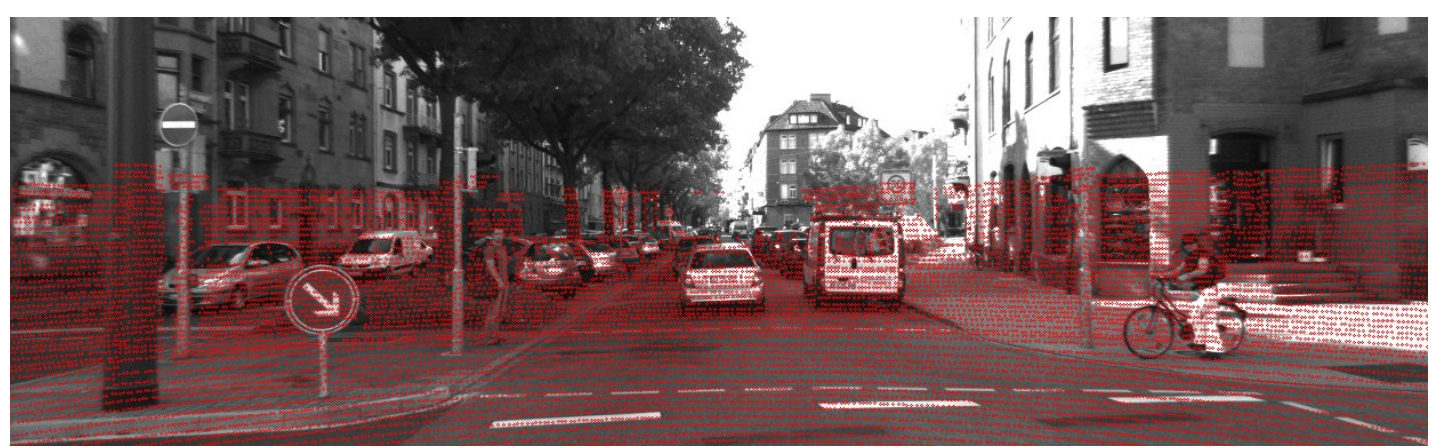

(a)

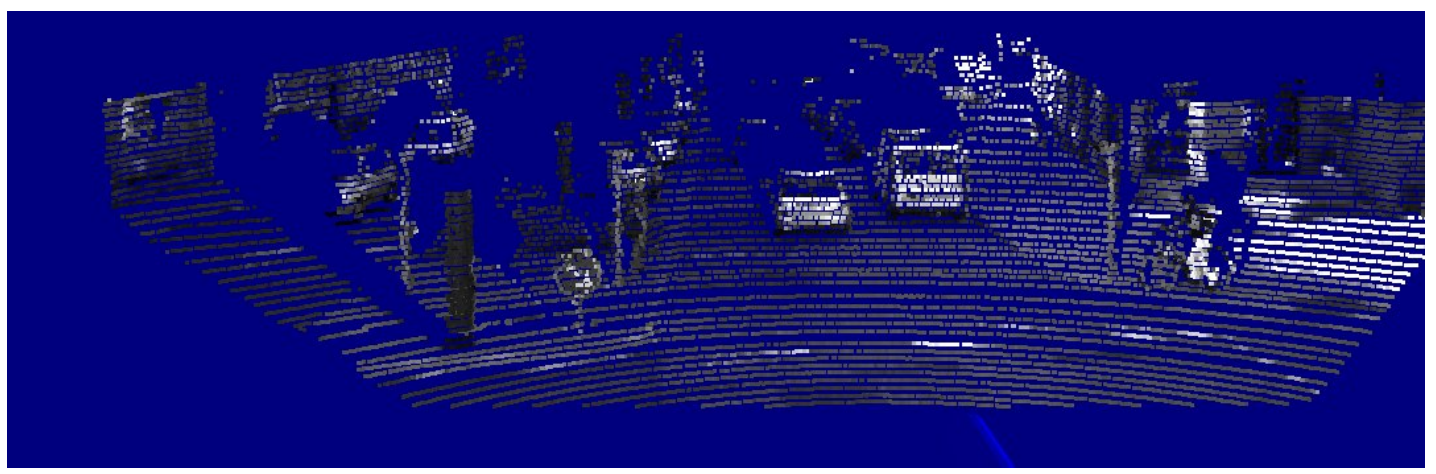

(b)

Figure 3.5: Upper image: 3D points are showed in red using the $(u, v)$ image coordinates obtained by transformation from matrices calibrations. Bottom image: Point cloud where each point is coloured (gray-scale) with the respectively pixel from the image.

Before running stereo matching algorithm, we apply a histogram equalization method to both images (left and right) to smooth noise from heavy shadows. We also select a region of interest $(r o i)$ with (height $=\frac{2}{3}$ original_height) to improve the speed performance and the disparity image is then computed. In contrast with several currently applications, we do not make use of post-processing techniques like median filter or bilateral filter that tries to create a fully dense disparity map. Since our obstacle estimation method works with a sparse set of points $P$, after computing the disparity map, our method selects pixels equally distributed in the image. In other words, the set $P$ contains only points $p=(u, v, d)$ where $d$ is a valid disparity value (greater than zero), and given a constant $\lambda$ and any $i$, we select only points with $u=(\lambda * i)$ while $(u<w i d t h)$, and $v=(\lambda * i)$ while $(v<h e i g h t)$ (see an example in Figure 3.7a). Due the characteristic of our method, we also propose a modification of ELAS to improve the runtime. This modification is the calculation of disparity value only in the lines $v=(\lambda * i)$, i.e. only lines that have points which the obstacle estimation method will use. Figure $3.7 \mathrm{~b}$ shows the result of stereo correspondence algorithm limited to lines used by the obstacle estimation method. 


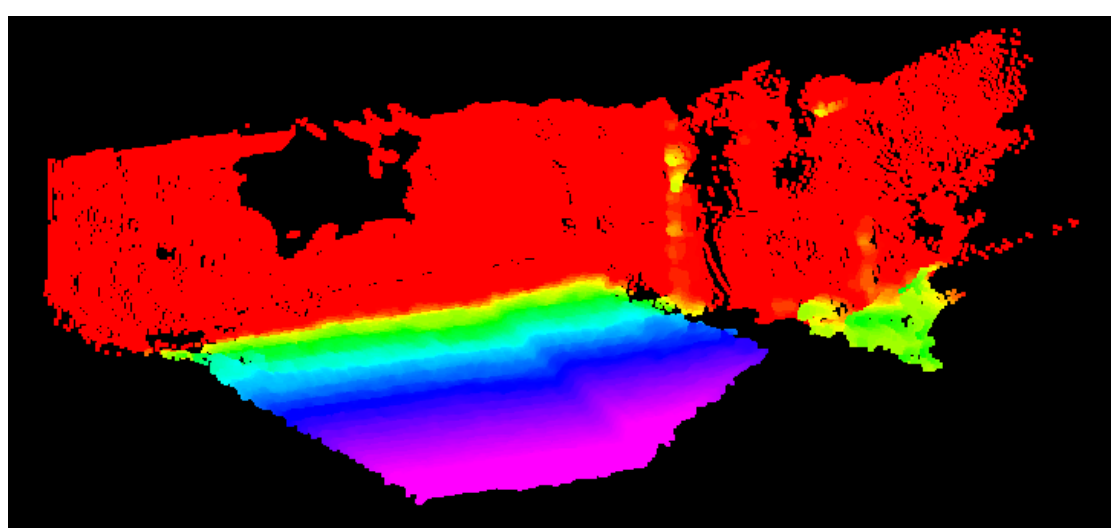

(a) 3D point cloud showing disparity values

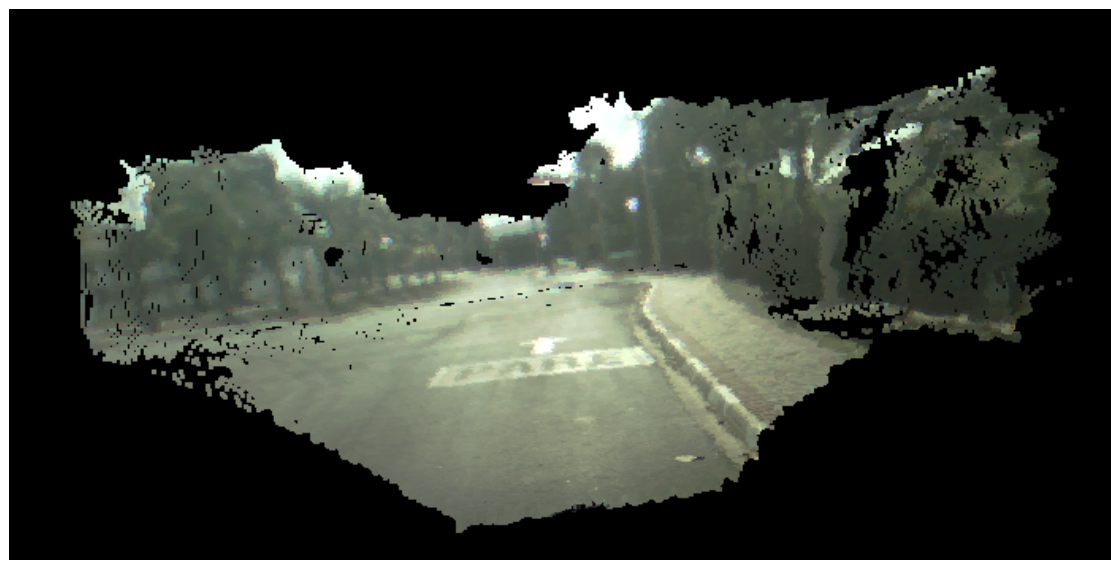

(b) 3D point cloud showing RGB values

Figure 3.6: Results of reprojection step of a stereo image sensor.

\subsection{Estimation of Obstacles in Image Plane}

Given the definition of obstacle by the spatial-condition and a generic modelling of a 3D point cloud in graph format, the method of estimation of obstacles is resumed to a calculation of a cost value $C$ for each node $p$ from graph $G$, where $0.0 \leq C \leq 1.0$ and represents a confidence level of node $p$ being classified as an obstacle. Using graph $G$ generated by a pre-processing step, our approach calculates $C$ using Equation 3.5, which depends only on the local spatial condition represented by Equation 3.1 for each pair of nodes connected by an edge. This method classifies nodes as obstacles without any constant height threshold value. Figure 3.8 shows an example of the confidence level of nodes in the screen plane. The image is a sample of information data used to generate the road confidence map.

Given graph $G=\{P, E\}$, for each node $p_{1} \in P$ there exists a subset $Q\left(p_{1}\right) \subset P$ of nodes that represents the neighbourhood of $p_{1}$. The cost value $C$ from node $p_{1}$ is calculated as in 


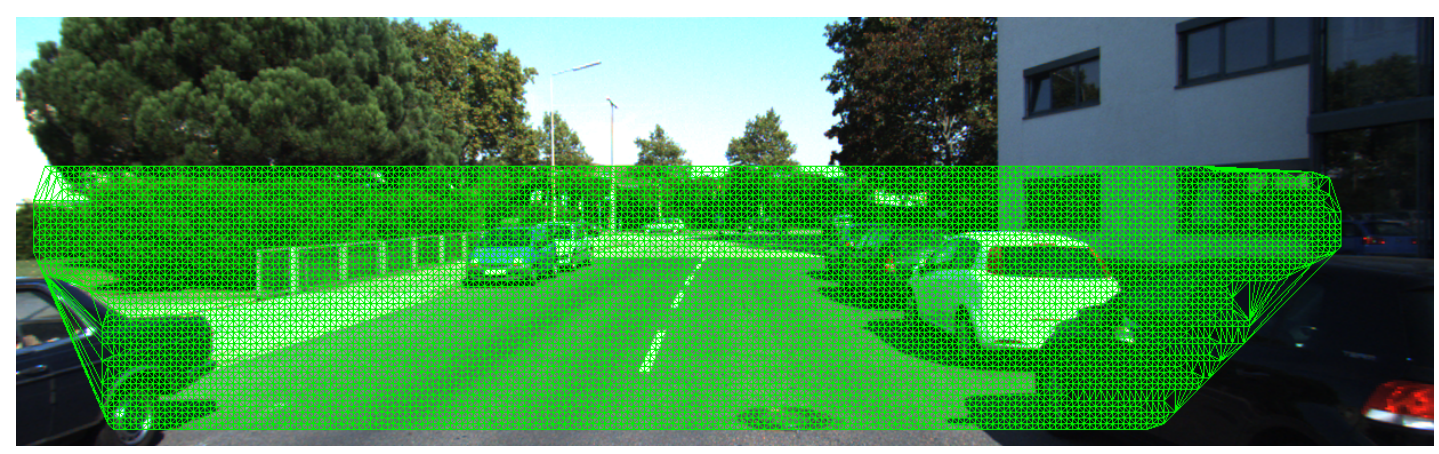

(a) Sparse set of points and edges projected in image.

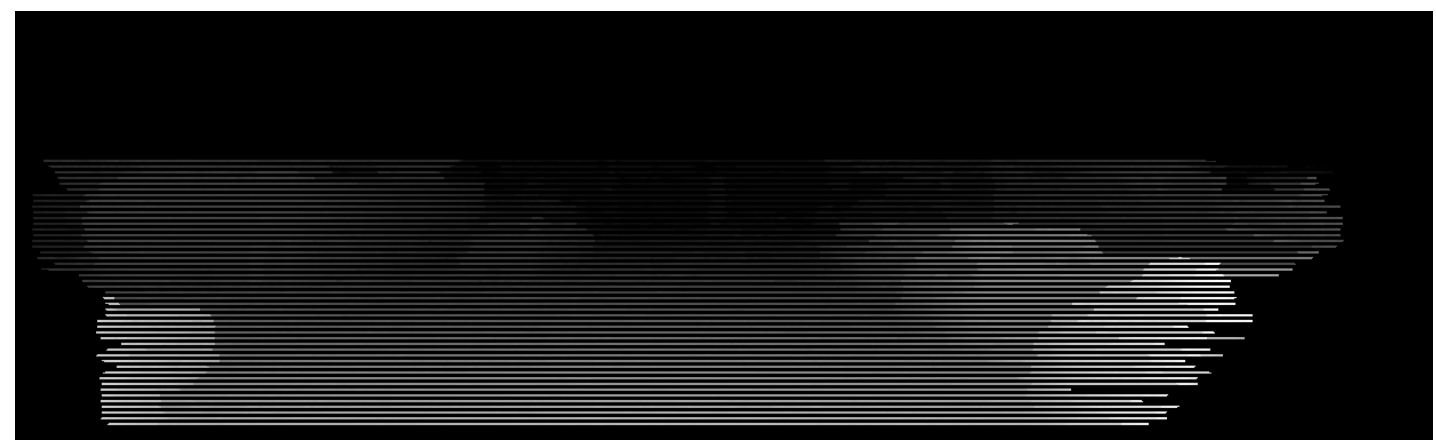

(b) Sparse Disparity Map

Figure 3.7: Sparse 3D points generated by stereo image sensors and projected in left image.

Equation 3.5:

$$
C\left(p_{1}\right)=\frac{1}{\left\|Q\left(p_{1}\right)\right\|} \sum_{n \in Q\left(p_{1}\right)} \text { sigmoid }\left(\frac{\left|p_{1 y}-p_{2 y}\right|}{\| p_{1}-p_{2}||}, \cos (\theta), \sigma\right)
$$

where $\left|p_{1 y}-p_{2 y}\right|$ corresponds to the absolute difference of height values between these two points, $\left\|p_{1}-p_{2}\right\|$ is the euclidean distance between points $p_{1}$ and $p_{2}, \theta$ and $\sigma$ are suitably chosen constants, $Q\left(p_{1}\right)$ can be expressed as $Q 1\left(p_{1}\right) \cup Q 2\left(p_{1}\right)$, where $Q 1\left(p_{1}\right)=\left\{p \mid\left(p_{1}, p\right) \in E\right\}$ and $Q 2\left(p_{1}\right)=\left\{p \mid\left(p_{2}, p\right) \in E \wedge p_{2} \in Q 1\left(p_{1}\right)\right\}$, and $\operatorname{sigmoid}(x, \mu, \sigma)$ is a function defined as in Equation 3.2.

Our approach, which calculates continuous values as output from the obstacle estimation method was introduced to classify points from stereo image sensors (due the imprecision of disparity maps). Given the camera coordinate axes ( $x=$ right, $y=$ down, $z=$ front $)$, the general idea is: if value $\left(\frac{|p(y)-n(y)|}{\| p-n||}\right)$ is greater than a predetermined value $(\cos (\theta))$, then node $p$ is more likely to be classified as an obstacle. The average of all continuous values calculated for each neighbour of $p$ is the confidence degree of $p$ being classified as an obstacle, i.e. $C(p)$ is the obstacle-confidence of node $p$. 


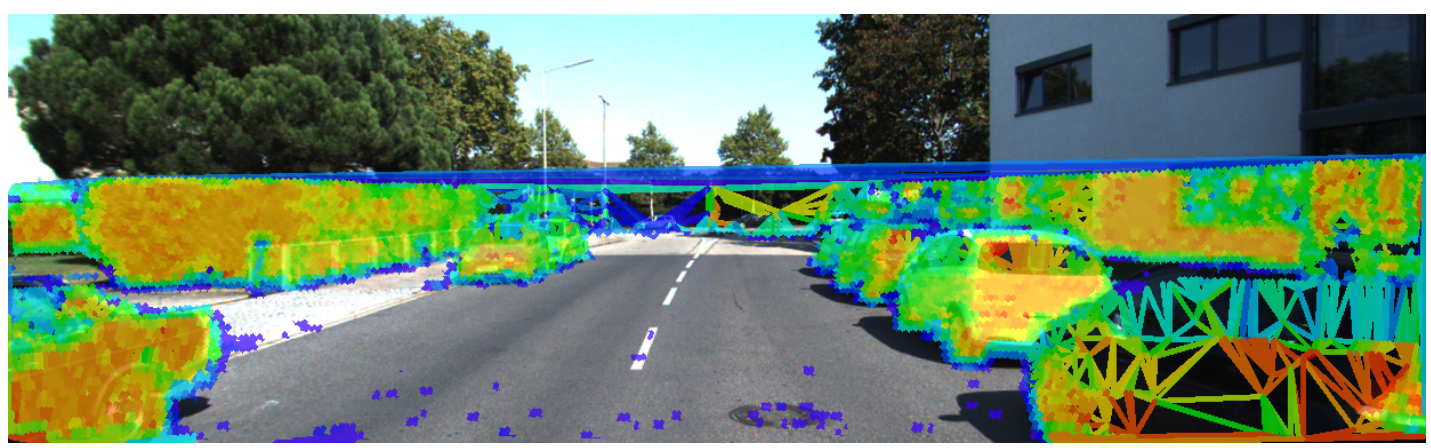

(a) Results with 3D LIDAR+Monocular Image Sensor. The large are on the road does not show color because the high precision of 3D-lidar generates cost $C=0$ for these points.

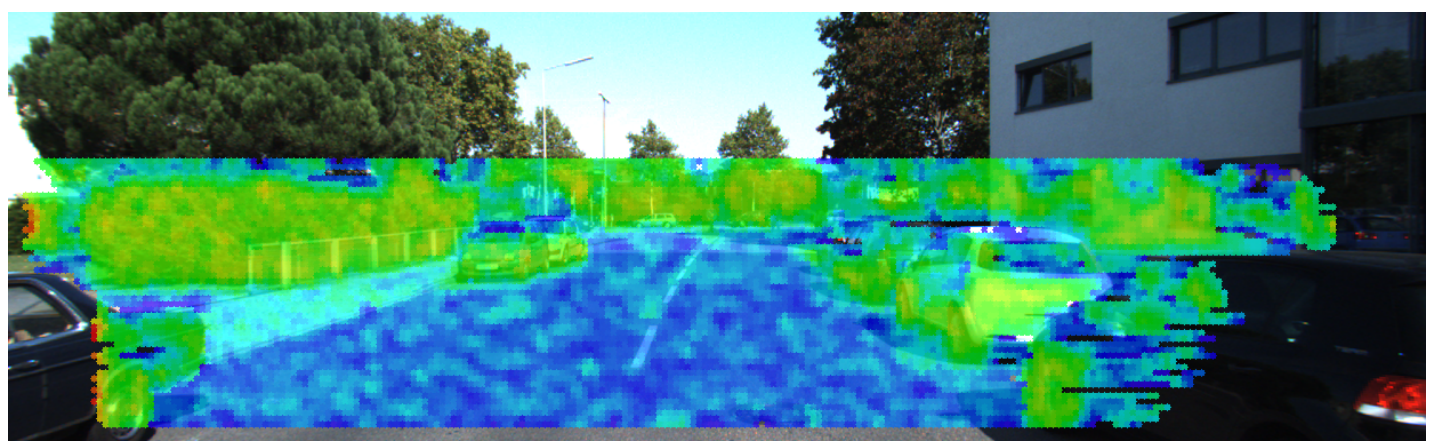

(b) Results with Stereo Image Sensor

Figure 3.8: Results from our obstacle probability calculation method in a 2D image world. Warmer color pixels indicate more confidence level of this pixel being an obstacle.

\subsection{Considerations}

Since our obstacle estimation method does not have a constant height threshold, with the same parameters is possible to detect several different types of obstacles like tall grass, trees, cars, pedestrians and even curbs. However, this method is directly dependant of the precision of the 3D point cloud to have a good performance. This dependency is evident in Figure 3.8 when we compare a single result from a stereo image approach versus a 3D-LIDAR and monocular image approach. To handle sensor noise and inaccuracy from correspondence algorithms, the road estimation method must consider several possible values that indicates $C(p)$ is an obstacle. 
This chapter presents a method to estimate road area in a screen plane (image) using the previously calculated obstacle-confidence of several pixels represented by nodes of graph $G$ (see chapter 3 for details about graph $G$ ). To handle sensor noise and the inaccuracy of stereo correspondence algorithms, the final result of our method is composed by a combination of several road-areas estimated by our method with minimal differences between the intern parameters. To improve robustness of our road estimation, and since it is an outdoor application, it is fair enough to take advantage of colors, assuming that green color means the grass that also delimits roads (section 4.2). Incorporating the green-confidence with the obstacle-confidence our system was able to handle situations where the road is not delimited by curbs, and instead, delimited by grass.

\subsection{Perspective Modelling of Road Detection Problem}

Since our obstacle estimation method does not have a constant height threshold (it is a spatial condition), with the same parameters it is possible to detect several different types of obstacles like tall grass, trees, cars, pedestrians and even curbs. Due to this, our road detection method estimates the road area assuming that "any road is delimited by the detected obstacles in the screen plane, the vehicle platform is already on the road, and the vehicle is not upside down". This estimation is computed based on the combination of several road boundaries represented 
by polygons, where each one is generated from a structure called polar-range-histogram (same intuition of vector field histogram (Borenstein e Koren, 1991)). The major difference between these histograms is that each one has a different origin point and/or a different threshold value $\beta$. The general idea of $\beta$ is that, using the previously calculated cost value $C(p)$ (chapter 3 ), a node $p$ is classified as an obstacle if and only if $C(p)$ is higher than $\beta$. Therefore, many road boundary candidates can be created using soft and severe threshold values. The assumptions "vehicle platform is already on the road and vehicle is not upside down" allow us to define the origin points in bottom central area of screen plane and avoid geometric shape assumptions.

\subsubsection{Polygon Generation}

Before the generation of a polygon, the respective polar-range-histogram must be computed. In this step, a polar histogram $H_{\text {hist }}$ is defined by one origin-point $(u, v)$ located in central bottom area of the image, a threshold value $\beta$ for the obstacle classification and a number of bins $k$. The number of bins is defined as $k=\left(360^{\circ} /\right.$ precision-degree $)$, where precision-degree is a suitably chosen constant. Is important to notice that the choice of this parameter directly influences the runtime (more bins means more processing time) and the accuracy of detection of road area (more bins can improve the hit rate), a discussion about these influences is given in section 5.2.

Given a polar-range-histogram $H_{\text {hist }}$, the amplitude of each bin is the $2 \mathrm{D}$ euclidean distance between origin-point $(u, v)$ and the closest obstacle-node $(u, v)$ in the respective angle. See an example of this histogram in Figure 4.1a in which origin-point $(u, v)$ is showed in red color, and all amplitudes are shown in green lines. Without loss of generality, a polygon $H_{\text {poly }}$ (Figure $4.1 \mathrm{~b}$ ) is a set of all $2 \mathrm{D}$ points derived from the projection of all bins of $H_{\text {hist }}$ in the screen plane as shown the Equation 4.1. The images in Figure 4.1 show, respectively, the polar range histogram and the polygon that represents one of estimations of the road area with arbitrary parameters.

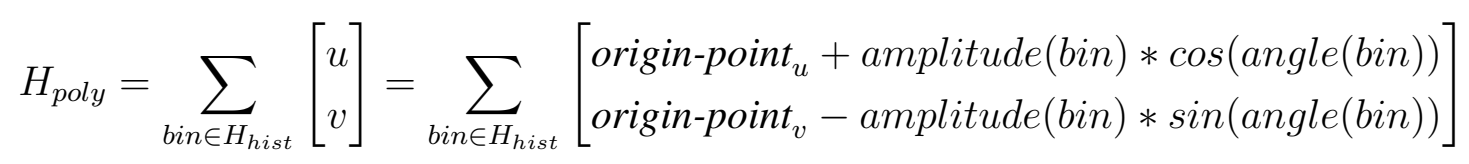

where amplitude() and angle() are functions that returns, respectively, the euclidean distance and the angle stored in a bin from $H_{\text {hist }}$.

\subsubsection{Estimation of Road Area}

Sensor noise is a very common problem of several robotic applications, even highly-precise and costly sensors has some noise in particular situations, e.g., distance calculated by a LI- 


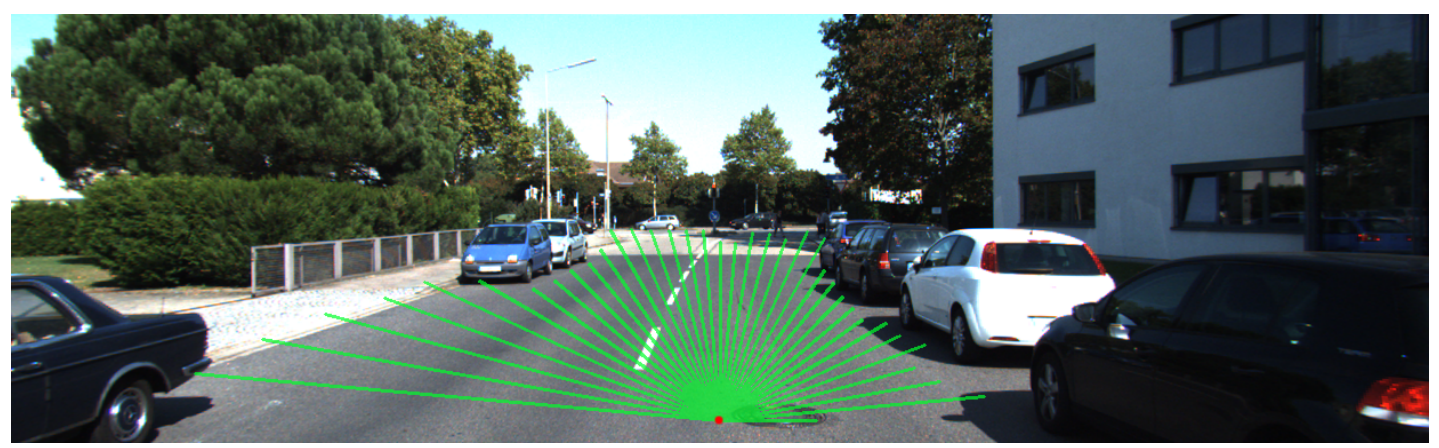

(a) Histogram with $k=36$ bins, i.e. (precision-degree $=10^{\circ}$ ). The red point is the origin-point

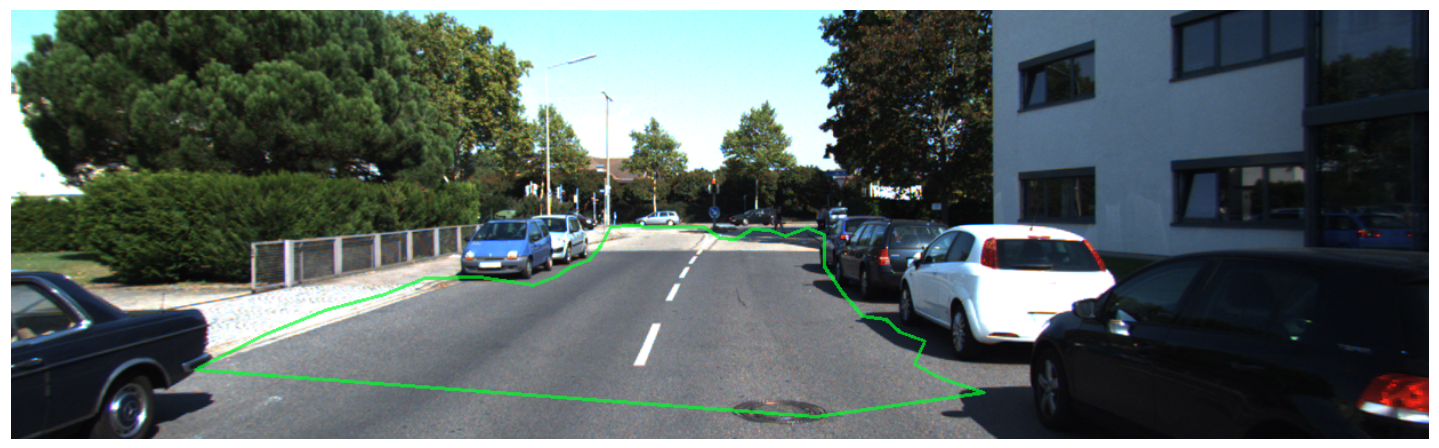

(b) Polygon with $k$ vertex.

Figure 4.1: Comparison between the data structure of one polygon and a polar histogram that estimates road area.

DAR sensor in a specular/black surface. Stereo image sensors are more susceptible to noise over long distances due the size of camera sensor (CCD or CMOS), and easily suffer noise due to lighting conditions. To handle noise over the 3D coordinates and consequently, over obstacle-estimation, the last part of the proposed road estimation method is a generation of a confidence degree $R$ for each pixel from the screen plane. This confidence degree is calculated by combination of an arbitrary set $H$ of polygons by the function described in Equation 4.2 which calculates $R(p)$ for each pixel $p$ inside the screen plane. Fig. 4.2 shows a sample of a road estimation result from our proposed method in thermal-color style images (Red color means highest confidence level while blue means the lowest confidence level).

$$
R(\text { pixel })=\sum_{h \in H} \begin{cases}\frac{1}{\|H\|} & \text { if pixel is inside polygon } h \\ 0 & \text { otherwise }\end{cases}
$$

where $\|H\|$ is the number of estimated polygons.

In order to maintain the low runtime and depending of the required output, this method also can be modified with changing "estimation of every pixel" by "estimation of each node $p$ of $G$ ". 


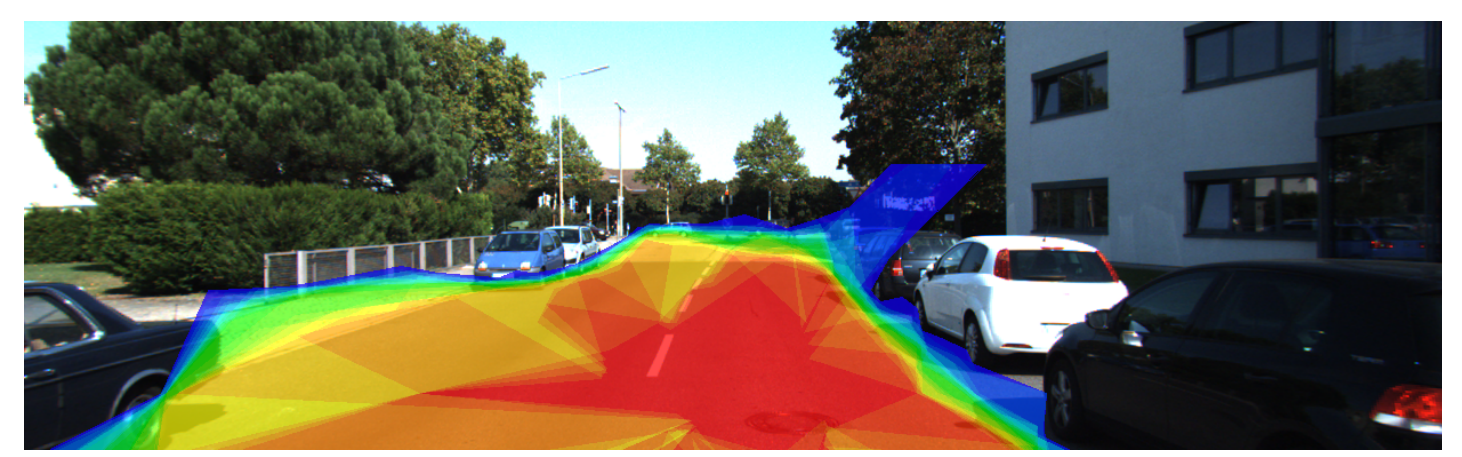

Figure 4.2: Result of Estimation of road shape with several thresholds and origin points.

In this case, a "Point-in-Polygon" method of complexity $n \log (n)$ is used to compute $R(p)$, and since $0 \leq R(p) \leq 1.0$, these values can be interpolated to propagate the classification values for all pixels of an image without nodes in graph $G$. The result of this approach is showed as an image in Fig. 4.3. Such a method was not used in our study because the required output to evaluate is the perspective image, so the estimation of every pixel is more suitable.

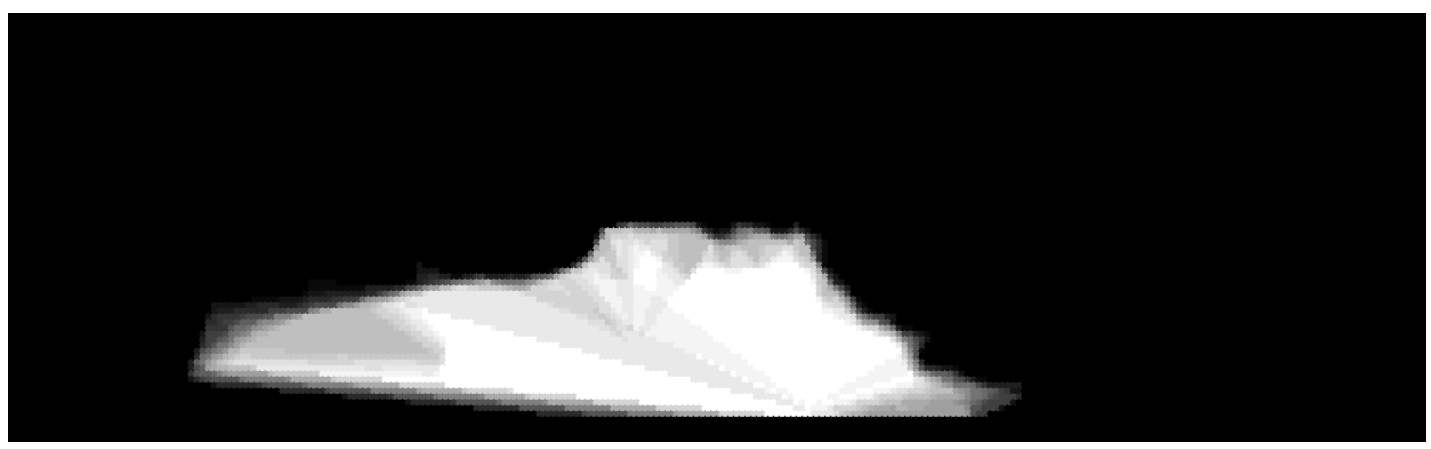

Figure 4.3: Result of our road estimation method as "estimation of each node $p$ of $G$ ". The $R(p)$ is propagate for all pixels neighbours of $p$ that are inside of an arbitrary radius of distance.

In this work, the road detection method generates a road-confidence in screen planes. This method is not dependant of road markings, color-models, shape or geometric features as flat surface and height. It uses continuous values (obstacle-confidence) as input to estimate a continuous confidence map as Figure 4.3 that can be transformed to real 3D space by reconstruction of stereo system or return to 3D-LIDAR coordinate frame to create 2D/3D maps commonly used by path-planning algorithms available on literature. 


\subsection{Improvements with Green-Confidence}

In order to take advantage of the colors already available in the screen planes derived from cameras, this work also presents a processing method to calculates how likely a particular pixel is green (green-confidence). The motivation behind the use of this detector is that common roads (most of time, out-city roads) sometimes does not have curbs to delimit them, and instead, they have grass shortly after coasting of road (see Figure 4.4 for a sample). Therefore, the green-confidence can be combined with obstacle-confidence to create a more robust road estimation method. Another advantage about this green-confidence is that the green color is the most representative color in the Chromaticity Diagram ${ }^{1}$, i.e. it is a color with a larger range of combinations.
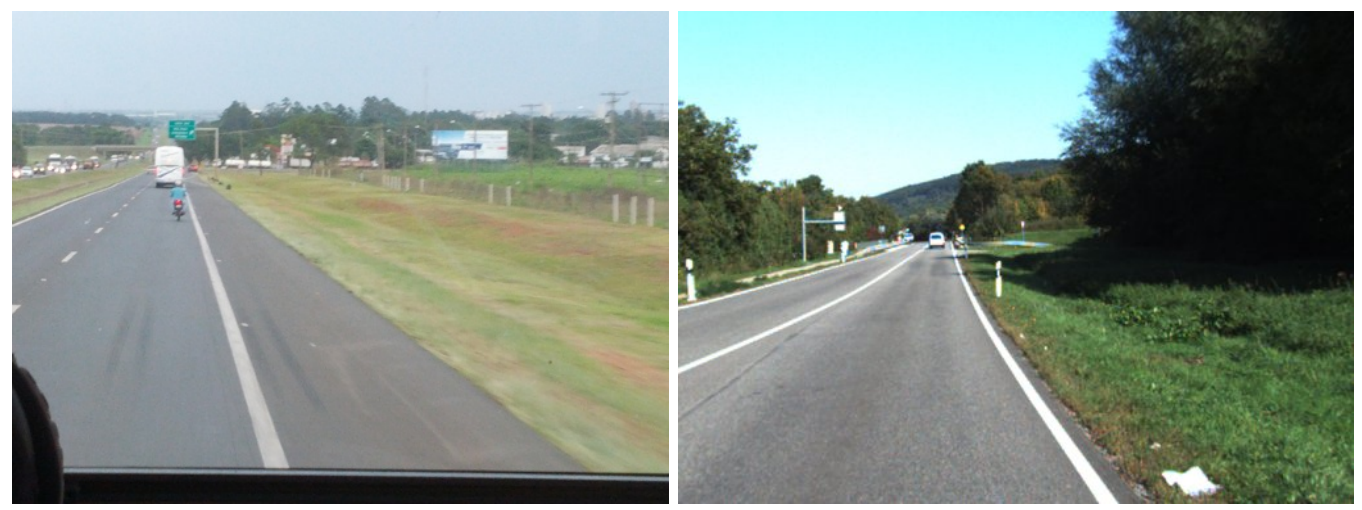

Figure 4.4: Out-city road without curbs but with grass as delimiter of the road area.

The $\mathrm{L}^{*} \mathrm{a} * \mathrm{~b} *$ color space $\left(\mathrm{CIELAB}^{2}\right)$ is a color space independent of device, includes all perceivable colors (gamut exceeds RGB model), and also minimizes redundancy between the colors. Color spaces like HSV, HSL and RGB, have a lot of redundancy in cases with strong light effects (any color can be gray depending of saturation value). The three coordinates of CIELAB represent the lightness of the color $\left(\mathrm{L}^{*}=0\right.$ yields black and $\mathrm{L}^{*}=100$ indicates white), its position between red/magenta and green $\left(\mathrm{a}^{*}\right.$, negative values indicate green while positive values indicate magenta) and its position between yellow and blue ( $b^{*}$, negative values indicate blue and positive values indicate yellow). Figure 4.5 shows the RGB color space projected into $\mathrm{L}^{*} \mathrm{a} \mathrm{b}^{*}$ space with several different $\mathrm{L}^{*}$ values, $\mathrm{a}^{*}$ flows in the horizontal line (left-to-right), and $\mathrm{b}^{*}$ in the vertical line (bottom-to-up), both lines varying from -127 to +127 .

\footnotetext{
${ }^{1}$ Chromaticity Diagram is two-dimentional diagram in which points specified by chromaticity coordinates represent the chromaticities of color stimuli.

${ }^{2} \mathrm{CIE} \mathrm{L} * \mathrm{a} * \mathrm{~b} *(\mathrm{CIELAB})$ is a color space specified by the International Commission on Illumination(CIE, 2014)
} 


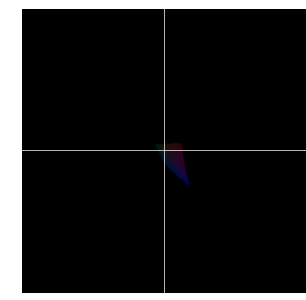

(a) $\mathrm{L}^{*}=5$

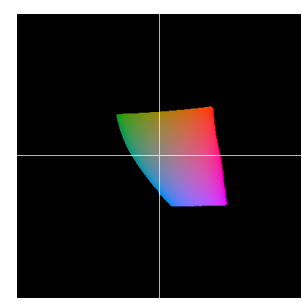

(f) $\mathrm{L}^{*}=55$

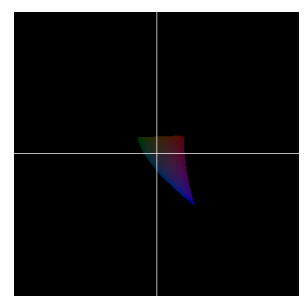

(b) $\mathrm{L}^{*}=15$

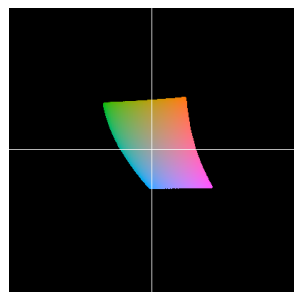

(g) $\mathrm{L}^{*}=65$

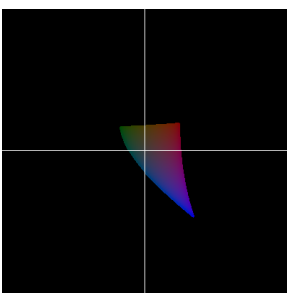

(c) $\mathrm{L}^{*}=25$

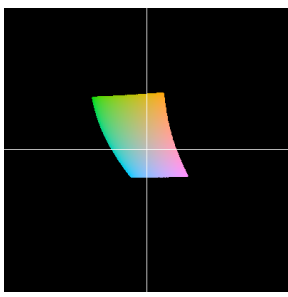

(h) $\mathrm{L}^{*}=75$

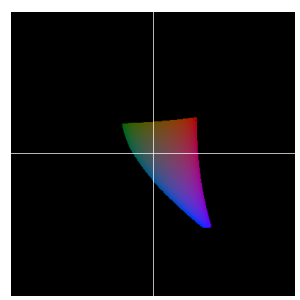

(d) $\mathrm{L}^{*}=35$

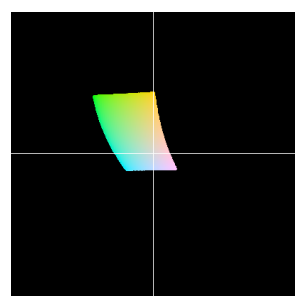

(i) $\mathrm{L}^{*}=85$

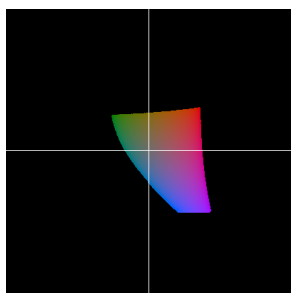

(e) $\mathrm{L}^{*}=45$

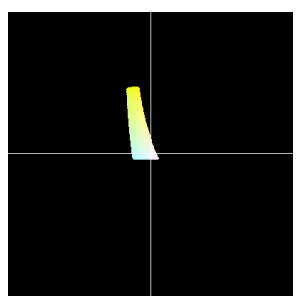

(j) $\mathrm{L}^{*}=95$

Figure 4.5: $\mathrm{L} * \mathrm{a} * \mathrm{~b} *$ color space showing the $\left(\mathrm{a}^{*} \mathrm{~b}\right)$-space with different $\mathrm{L} *$ values. The darker colors are more likely to be blue than green/red. Coordinate $a^{*}$ flows in the horizontal line, and coordinate $b^{*}$ in the vertical line.

In $\mathrm{L}^{*} \mathrm{a} * \mathrm{~b} *$ color space, due to the fact that the green color is located in the upper left quadrant, any pixel that has $\left(b^{*}<0\right)$ or $\left(a^{*}>0\right)$ are ignored, i.e. their green-confidence are zero. Furthermore, any pixel with $\left(\mathrm{L}^{*}>90\right)$ is also ignored to avoid colors with high lightness (white color from road markings). The green-confidence of remaining pixels are computed by a $2 \mathrm{D}$ euclidean distance in the $\left(\mathrm{a}^{*} \mathrm{~b}^{*}\right)$-plane between the green-point $g$ and pixel $p$. Note that $g$ must represent the green-color, due to this $g$ is located in the central area of the upper left quadrant of $\mathrm{a}^{*} \mathrm{~b}^{*}$-plane. Equation 4.3 shows how compute the green-confidence of each pixel $p$.

$$
G(p)=1.0-\left(\frac{\sqrt{\left(p_{a *}-g_{a *}\right)^{2}+\left(p_{b *}-g_{b *}\right)^{2}}}{\sqrt{\left(\text { max_dist }_{a *}\right)^{2}+\left(\text { max_dist }_{b *}\right)^{2}}}\right)
$$

where $\left(g_{a *}=-64\right),\left(g_{b *}=+64\right)$, because $g$ is the central point of upper left quadrant and since other quadrants are ignored, max $\left._{-} d i s t_{a *}=\max _{-} d i s t_{b *}=64\right)$.

In order to maintain compatibility with the obstacle-estimation method, $p$ is a node and the $\left(\mathrm{L}^{*}, \mathrm{a}^{*}, \mathrm{~b}^{*}\right)$ values are calculated after performing a median blur filter in the RGB image. Without loss of generality, all possible euclidean distances calculated in Equation 4.3 always are smaller than 1.0, so $G(p)$ always will be smaller than 1.0. The result of computation of the green-confidence is showed in Figure 4.6 where only green nodes are highlighted, warmer colors represents greater confidence about node $p$ be green and therefore, be the grass that 


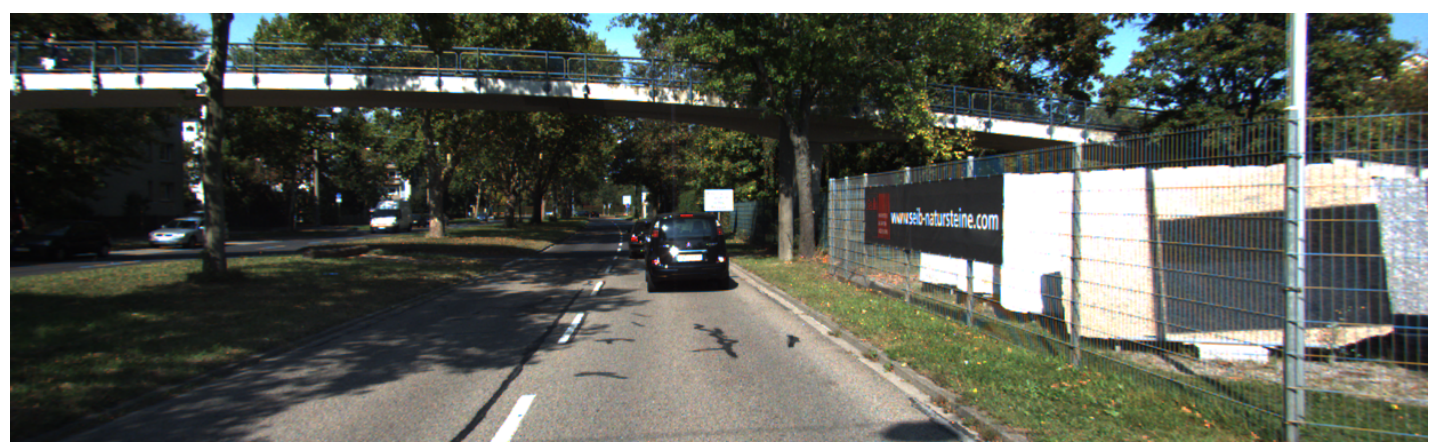

(a) RGB image

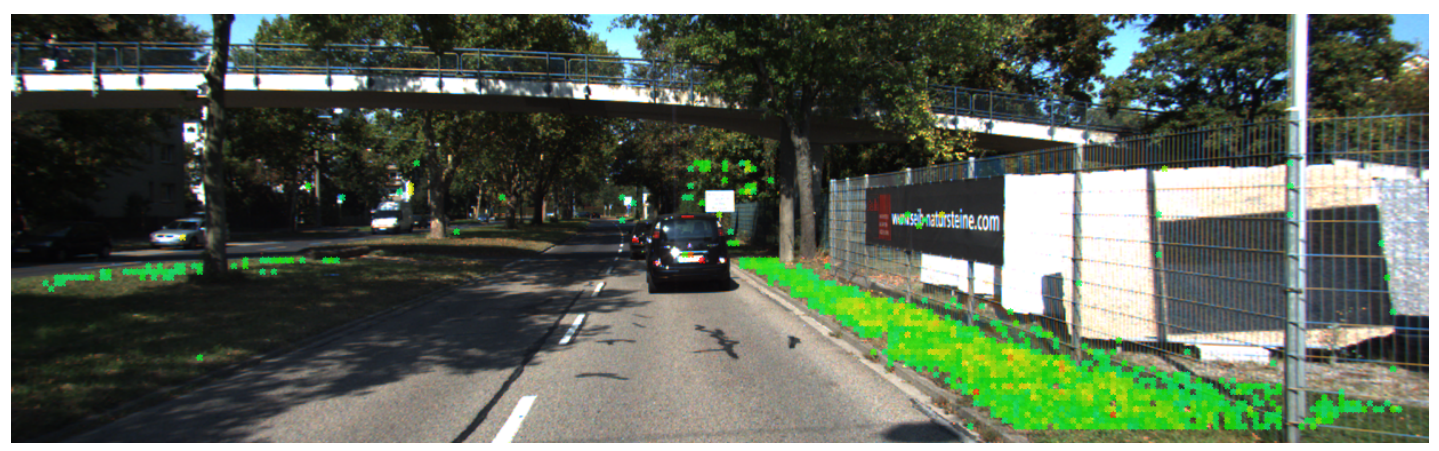

(b) Green Estimation

Figure 4.6: Result of our green detection method, warmer colors represents greater confidence about node $p$ be the grass that limits the road. Is possible to see that darker green does not have a good confidence since is too close to the limits of the upper left quadrant, i.e. too close of darker colors in the $\mathrm{L}^{*} \mathrm{a}^{*} \mathrm{~b} *$ space.

limits the road. It is possible to see that darker green does not have a good confidence since it is too close to the limits of the upper left quadrant, i.e. too close to darker colors from other quadrants in the $\mathrm{L}^{*} \mathrm{a} * \mathrm{~b}^{*}$ space. If $G(p)>$ green_threshold, our system incorporates this confidence to obstacle-confidence as described in Equation 4.4 before compute the road boundaries. Figure 4.7 shows obstacle-confidence alone and obstacle-confidence combined with green-confidence. It is easy to see a improvement in the grass area with good light conditions, i.e. outside of dark shadows areas on the left part of image.

$$
C(p)= \begin{cases}\min ((C(p)+G(p)), 1.0) & \text { if } G(p)>\text { green_threshold } \\ C(p) & \text { otherwise }\end{cases}
$$

Since it is an outdoor application, it is fair to make the assumption that green color means the grass that also delimits the road. Incorporating a green-confidence with the obstacle-confidence our system is able to handle situations where the road is not delimited by curbs, and instead, delimited by grass. Since this situation occurs only occasionally, its improvement is quite low 


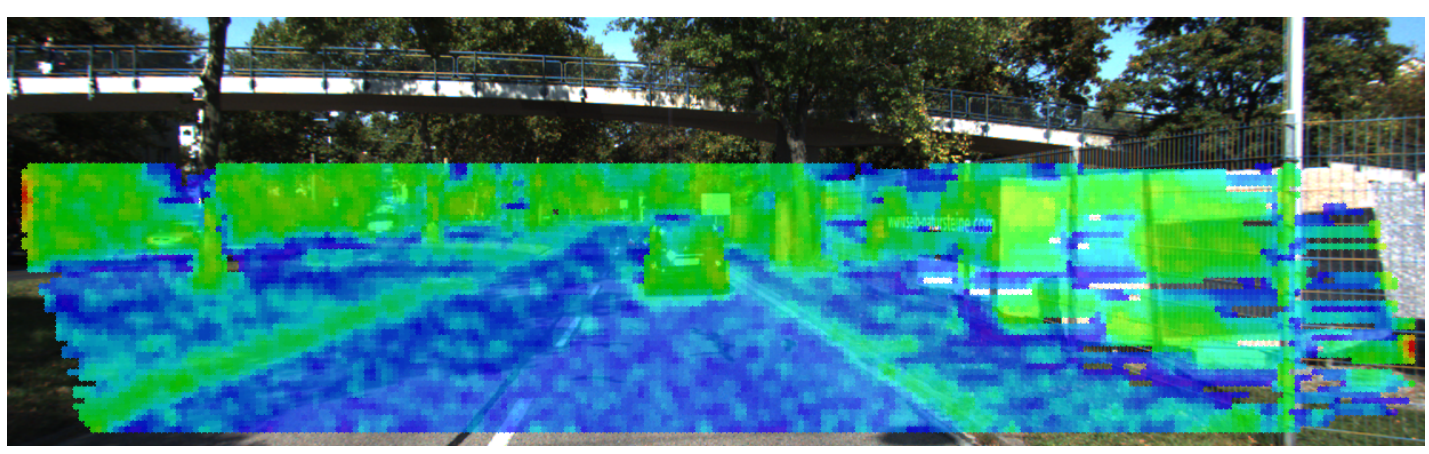

(a) Obstacle Confidence

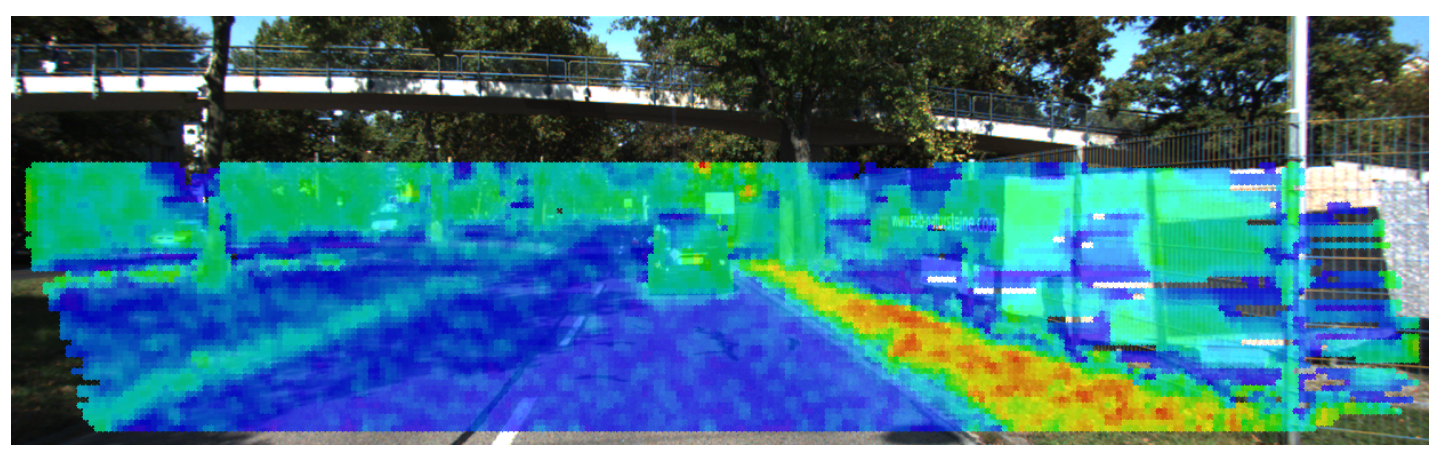

(b) Obstacle Confidence+Green Confidence

Figure 4.7: Result of combination of green-confidence with obstacle-confidence, warmer colors represents greater confidence about node $p$ be obstacle/grass that limits the road. Is easy to see a improvement in the grass area with good light conditions.

but as runtime of this method is very low, its use is recommended for intelligent vehicles applications even in road scenarios.

\subsection{Considerations}

This chapter presented a method to estimate the road area using the obstacle-confidence detailed in previous chapter. This chapter also presented a method to create a "grass-confidence" to improve the road area estimation. This method uses $\mathrm{L}^{*} \mathrm{a}^{*} \mathrm{~b}^{*}$ colorspace to take advantage of structured separation of green color from others. In general, to handle sensor noise and inaccuracy from any processing step, all methods generates continuous values as confidence maps. Furthermore, the majority of these methods avoid several assumptions and uses a reduced number of parameters to work. 


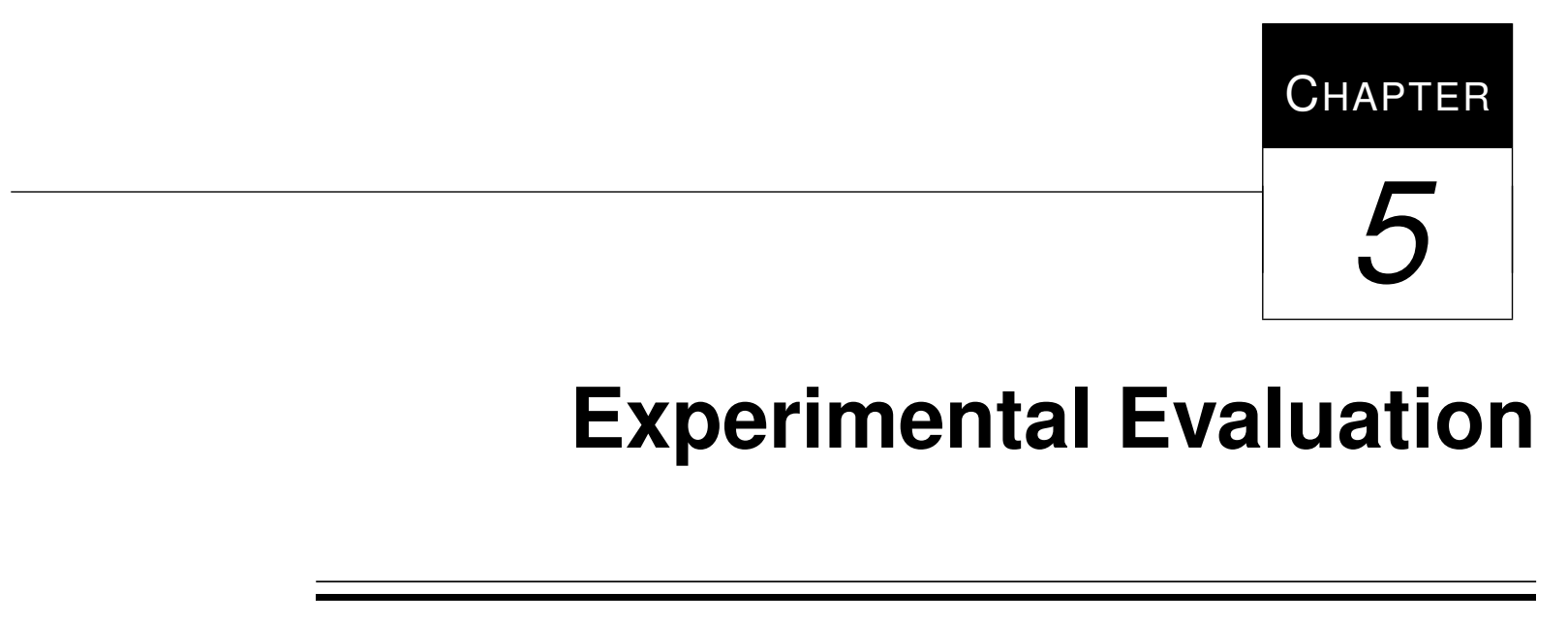

This chapter presents several experiments using a public available dataset to evaluate and compare the proposed algorithm with other current approaches over the world. It discuss some concepts about evaluation metrics, the ground-truth quality and how the choice of parameters influences the accuracy of results. This chapter also describes results of the proposed algorithm with different sensor systems to show the non-dependence of specific sensors, i.e. the proposed algorithm can be used by several 3D sensors, like stereo cameras, kinect, and combinations of LIDARs or 3D-LIDARs.

In order to validate the road estimation method described in the previous chapter, we used experimental data from the AnnieWAY platform available online on KITTI Benchmark (Geiger et al., 2013, 2012a). More specifically we used the KITTI-ROAD Dataset (Fritsch et al., 2013), which includes calibration parameters, ground-truth images, and scripts for evaluation of road detection methods. We adopted the same evaluation of Fritsch et al. (2013) which verifies the road area in a 2D metric space. Therefore, we transformed the ground-truth images and our road estimation result data into bird-eye-view (BEV) image (Mallot et al., 1991) because it avoids the perspective effect that brings bias to the obstacles in a near range (see Figure 5.1 for a sample). The images in the BEV representation cover $-10 m$ to $10 m$ in the lateral direction and $6 \mathrm{~m}$ to $46 \mathrm{~m}$ in the longitudinal direction. The resolution used is 0.05 meters / pixel and the images have $800 \times 400$ pixels. 


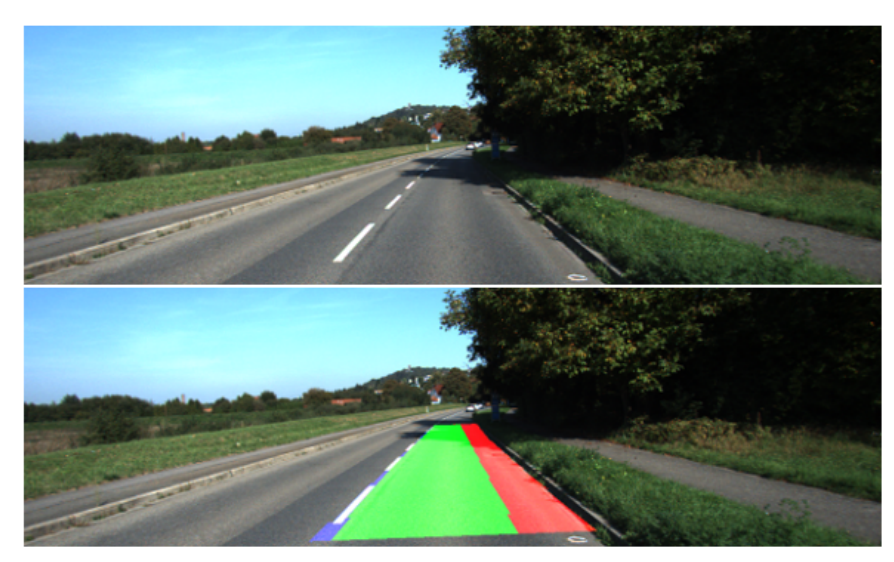

(a) Perspective Images
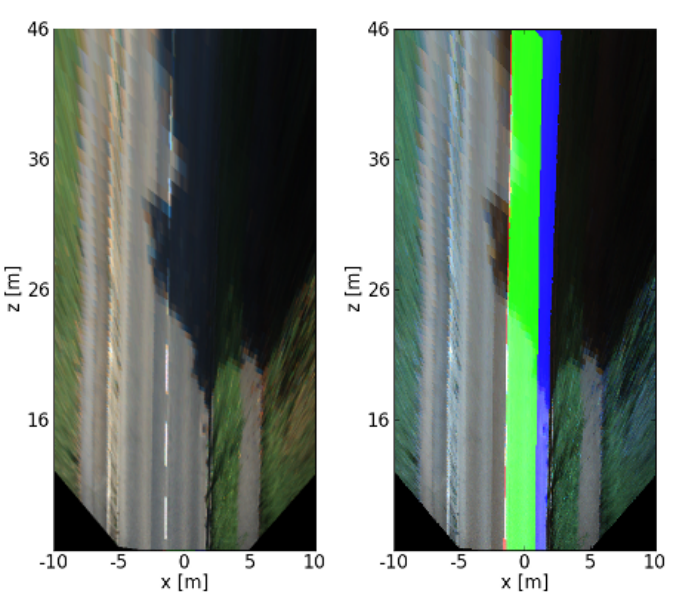

(b) Bird-eye-view Images

Figure 5.1: We adopted the same evaluation of (Fritsch et al., 2013) which evaluates the road area in a $2 \mathrm{D}$ metric space represented by bird-eye-view image (BEV). These samples of image results from a lane detection system shows the perspective effect that brings bias to pixels in near range (bottom part of image (a)), while in BEV images all pixels have the same weight in any classical pixel-based evaluation method.

Since most road detection approaches are based on supervised machine learning, the ROAD Benchmark is divided in two datasets. The first one is the training-dataset, which includes ground-truth images and the second one is a dataset that the evaluation is only available online ${ }^{1}$, i.e., with no externally available ground-truth. These datasets comprise three different categories, namely Urban Marked (UM), Urban Multiple Marked (UMM), Urban Unmarked (UU) and the scripts provided by (Fritsch et al., 2013) generate a specific score for each one. These scores are the classical pixel-based metric known as Precision, Recall and False Positive Rate (FPR) and False Negative Rate (FNR), and are computed after the transformation between image domain and BEV space. As the primary metric value to comparison, the KITTI-ROAD benchmark uses F1-measure (hamonic mean of Precision and Recall, with $\beta=1$ ) to rank all methods, also the $\operatorname{argmax}(\mathbf{F 1}$-measure) is used to select the best threshold of a confidence map in order to generate all classical scores (TP, TN, FP, FN) from a binary classification. In order to provide insights into the performance over the full Recall range, the Average Precision is computed for different Recall values $r$. The follow equations shows how compute all these scores:

$$
\begin{gathered}
\text { Precision }=\frac{T P}{T P+F P} \\
\text { Recall }=\frac{T P}{T P+F N}
\end{gathered}
$$




$$
\begin{gathered}
F 1-\text { measure }=\left(1+\beta^{2}\right) \frac{\text { PrecisionRecall }}{\beta^{2} \text { Precision }+ \text { Recall }} \\
\text { AveragePrecision }=\frac{1}{11} \sum_{r \in 0,0.1, \ldots, 1} \text { maxPrecision }(r)
\end{gathered}
$$

Where TP, FP and FN are, respectively, the number of classified pixels True Positive, False Positive and False Negative. As the approach presented in this thesis does not need training, we used the training-dataset to make a more detailed evaluation and discuss some particular cases. Our implementation was written in C++ with optimizations using OpenMP library(Dagum e Menon, 1998) and evaluated in a machine with Intel(R) Core(TM) i7-2640M CPU @ 2.80GHz and with 8Gb DDR3, running Ubuntu 12.0464 bits.

As previously mentioned, this thesis presents a method that can be performed using several different sensors. Therefore, this chapter discuss the performance of our method with three different sensor setups, first is using 3D-LIDAR points projected in monocular image sensor (GRES3D+VELO), second is using stereo image sensor with a modified ELAS algorithm to compute disparity (GRES3D+SELAS), and finally third is using also stereo image sensor but with classical Semi-Global Block Matching algorithm that is available in OpenCV Library (Bradski e Kaehler, 2008) to perform the correspondence step (GRES3D+SGBM). Table 5.1 shows the chosen parameters of all approaches.

\begin{tabular}{|c|c|c|}
\hline Parameters & GRES3D+VELO & \begin{tabular}{l|l} 
GRES3D+SELAS & GRES3D+SGBM
\end{tabular} \\
\hline$\theta$ & $77^{\circ}$ & $11.7^{\circ}$ \\
\hline$\sigma$ & \multicolumn{2}{|r|}{50.0} \\
\hline$\lambda$ & not required & $5 p x$ \\
\hline roi & \multicolumn{2}{|r|}{ width $\times \frac{2}{3} h e i g h t$} \\
\hline green-threshold & \multicolumn{2}{|r|}{0.2} \\
\hline precision-degree & \multicolumn{2}{|r|}{$5^{\circ}$ (means 72 bins) } \\
\hline$\beta$ 's & \multicolumn{2}{|r|}{$0.08,0.10,0.12,0.14,0.16$} \\
\hline global-origin-point $g(u, v)$ & \multicolumn{2}{|r|}{ width $/ 2$, height -50} \\
\hline offsets in width of $g$ & \multicolumn{2}{|r|}{$-100,-50,0,+50,+100$} \\
\hline
\end{tabular}

Table 5.1: Chosen parameters in this experiment.

Even with different sensor approach, the reader can observe that only three parameters are different between them. The most important change is $\theta$, which enables the estimation of obstacles even in different 3D spaces, as UVD-space versus three-dimensional Euclidean space. Parameters $\lambda$ and roi are not required in GRES3D+VELO approach because there is no selection of points, since there are already sparse points inside the image due to the extrinsic matrix provided by a calibration method. The two last rows of Table 5.1 describes all origin-points used in the estimation of road area, these rows means given the global-origin-point $g(u, v)$ and 
the offsets (described in last row), all origin-points used to generate the polygons are these five points $(u-100, v),(u-50, v),(u, v),(u+50, v),(u+100, v)$. The follow sections discuss firstly the online evaluation and the runtime performance, followed by the evaluations using the training-dataset of GRES3D+VELO, GRES3D+SELAS and GRES3D+SGBM.

\subsection{Online KITTI-ROAD Benchmark}

The current result of our methods in general rank from the KITTI benchmark are showed in Table 5.2 and is possible see that GRES3D+VELO and GRES3D+SELAS belongs to the top ten group.

Table 5.2: Rank of road area estimation in online Kitti Benchmark (December,2014). MaxF is the maximum f1-measure.

\begin{tabular}{|c|c|c|c|c|c|c|c|c|}
\hline Rank & $\begin{array}{c}\text { Method } \\
\text { Environment }\end{array}$ & MaxF & $\mathbf{A P}$ & PRE & REC & FPR & FNR & Runtime \\
\hline 1 & $\begin{array}{c}\text { DDN (Mohan, 2014) } \\
\text { GPU @ 2.5 Ghz (Python + C/C++) }\end{array}$ & $92.55 \%$ & $89.34 \%$ & $94.62 \%$ & $90.58 \%$ & $2.97 \%$ & $9.42 \%$ & $2 \mathrm{~s}$ \\
\hline 2 & $\begin{array}{l}\text { ProbBoost (Vitor et al., 2014) } \\
>8 \text { cores @ } 3.0 \mathrm{Ghz}(\mathrm{C} / \mathrm{C}++)\end{array}$ & $87.21 \%$ & $77.79 \%$ & $86.96 \%$ & $87.47 \%$ & $7.55 \%$ & $12.53 \%$ & $2.5 \mathrm{~min}$ \\
\hline 3 & $\begin{array}{c}\text { SPRAY (Kuehnl et al., 2012) } \\
\text { NVIDIA GTX } 580 \text { (Python + OpenCL) }\end{array}$ & $86.33 \%$ & $90.91 \%$ & $86.78 \%$ & $85.89 \%$ & $7.53 \%$ & $14.11 \%$ & $45 \mathrm{~ms}$ \\
\hline 4 & $\begin{array}{c}\text { RES3D-Velo (Shinzato et al., 2014a) } \\
1 \text { core @ } 2.5 \mathrm{Ghz}(\mathrm{C} / \mathrm{C}++)\end{array}$ & $85.49 \%$ & $79.03 \%$ & $79.93 \%$ & $91.88 \%$ & $13.28 \%$ & $8.12 \%$ & $0.36 \mathrm{~s}$ \\
\hline 5 & $\begin{array}{c}\text { GRES3D+VELO } \\
4 \text { cores @ } 2.8 \mathrm{Ghz}(\mathrm{C} / \mathrm{C}++)\end{array}$ & $84.71 \%$ & $84.44 \%$ & $82.91 \%$ & $86.59 \%$ & $10.28 \%$ & $13.41 \%$ & $60 \mathrm{~ms}$ \\
\hline$\vdots$ & $:$ & : & $:$ & : & $:$ & $:$ & : & $\vdots$ \\
\hline 7 & $\begin{array}{c}\text { GRES3D+SELAS } \\
4 \text { cores @ } 2.8 \mathrm{Ghz}(\mathrm{C} / \mathrm{C}++)\end{array}$ & $83.94 \%$ & $86.44 \%$ & $81.15 \%$ & $86.93 \%$ & $11.62 \%$ & $13.07 \%$ & $110 \mathrm{~ms}$ \\
\hline$\vdots$ & $\vdots$ & & $:$ & $\vdots$ & : & $\vdots$ & $\cdot$ & $\vdots$ \\
\hline 11 & $\begin{array}{c}\text { GRES3D+SGBM } \\
4 \text { cores @ } 2.8 \mathrm{Ghz}(\mathrm{C} / \mathrm{C}++)\end{array}$ & $79.91 \%$ & $81.56 \%$ & $78.55 \%$ & $81.32 \%$ & $12.79 \%$ & $18.68 \%$ & $0.72 \mathrm{~s}$ \\
\hline$\vdots$ & $\vdots$ & : & : & $\vdots$ & : & : & : & $\vdots$ \\
\hline 13 & RES3D-Stereo (Shinzato et al., 2014b) & $79.91 \%$ & $81.56 \%$ & $78.55 \%$ & $81.32 \%$ & $12.79 \%$ & $18.68 \%$ & $0.7 \mathrm{~s}$ \\
\hline$\vdots$ & $\begin{array}{c}1 \text { core @ } 2.5 \mathrm{Ghz}(\mathrm{C} / \mathrm{C}++) \\
\vdots\end{array}$ & . & : & : & : & : & $\therefore$ & $\vdots$ \\
\hline
\end{tabular}

Despite our lower MaxF (if compared with the first approach), it is fair enough to highlight that among the approaches in the top ten group, our system is one of the fastest, losing only to (SPRAY)(Kuehnl et al., 2012) in which is an approach that makes use of GPGPU. Furthermore, it is important to notice that all the approaches (except ours) are based on a supervised machine learning techniques, i.e. all methods already has some kind of previous information (model of features) about the environment. A brief comparison between our current method (GRES3D) and our previous methods (RES3D-Velo (Shinzato et al., 2014a) and RES3D-Stereo (Shinzato et al., 2014b)) shows a significant improvement in our runtime which is $6 \times$ faster for the 
3D-LIDAR approach and almost $7 \times$ faster for the stereo approach. Such an improvement was achieved because our current approach does not require intermediate filters and does not compute disparity, neither classifies all the pixels, but only points selected to compose the graph. Furthermore, despite the previous approaches be based on the same idea of estimation, these systems does not have the same algorithm like current approach (GRES3D) in which changes only in the graph generation step making fairer the comparison between sensor systems (all GRES3D). All metric evaluations for each category of dataset proposed in (Fritsch et al., 2013) are shown in Table 5.3 and a graphical relation between Precision and Recall are shown in Figure 5.2. By default, all figures in this section show the GRES3D+VELO results in the first row, results of GRES3D+SELAS on the second row and finally, results of GRES3D+SGBM the third row. The score values were obtained by submitting our result images in the online website from KITTI benchmark.

Table 5.3: Road-area evaluation [\%] in a Testing Dataset. Online available

\begin{tabular}{|c|c|c|c|c|c|c|}
\hline Benchmark & MaxF & $\mathbf{A P}$ & PRE & REC & FPR & FNR \\
\hline \multicolumn{7}{|l|}{ GRES3D+VELO } \\
\hline UM_ROAD & $85.49 \%$ & $83.39 \%$ & $81.20 \%$ & $90.27 \%$ & $9.65 \%$ & $9.74 \%$ \\
\hline UMM_ROAD & $85.56 \%$ & $88.39 \%$ & $81.49 \%$ & $90.06 \%$ & $23.94 \%$ & $9.94 \%$ \\
\hline UU_ROAD & $83.78 \%$ & $80.48 \%$ & $81.39 \%$ & $86.31 \%$ & $6.57 \%$ & $13.69 \%$ \\
\hline URBAN_ROAD & $84.71 \%$ & $84.44 \%$ & $82.91 \%$ & $86.59 \%$ & $10.28 \%$ & $13.41 \%$ \\
\hline \multicolumn{7}{|l|}{ GRES3D+SELAS } \\
\hline UM_ROAD & $84.08 \%$ & $85.23 \%$ & $79.34 \%$ & $89.42 \%$ & $10.75 \%$ & $10.58 \%$ \\
\hline UMM_ROAD & $84.97 \%$ & $89.96 \%$ & $84.79 \%$ & $85.14 \%$ & $17.87 \%$ & $14.86 \%$ \\
\hline UU_ROAD & $82.31 \%$ & $84.08 \%$ & $77.06 \%$ & $88.32 \%$ & $8.75 \%$ & $11.68 \%$ \\
\hline URBAN_ROAD & $83.94 \%$ & $86.44 \%$ & $81.15 \%$ & $86.93 \%$ & $11.62 \%$ & $13.07 \%$ \\
\hline \multicolumn{7}{|l|}{ GRES3D+SGBM } \\
\hline UM_ROAD & $82.56 \%$ & $83.69 \%$ & $77.20 \%$ & $88.73 \%$ & $12.10 \%$ & $11.27 \%$ \\
\hline UMM_ROAD & $82.03 \%$ & $87.02 \%$ & $78.22 \%$ & $86.22 \%$ & $28.09 \%$ & $13.78 \%$ \\
\hline UU_ROAD & $81.73 \%$ & $81.69 \%$ & $78.92 \%$ & $84.76 \%$ & $7.53 \%$ & $15.24 \%$ \\
\hline URBAN_ROAD & $81.77 \%$ & $83.33 \%$ & $80.30 \%$ & $83.29 \%$ & $11.77 \%$ & $16.71 \%$ \\
\hline
\end{tabular}

The URBAN_ROAD line in Table 5.3 contains the average of all scores from all three categories. Since our system is free of geometric model assumptions, free of previous training, and all MaxF of URBAN line are higher than $80 \%$, the results from the road estimation can be considered satisfactory in comparison to other approaches in the literature and KITTI-ROAD benchmark. Furthermore, it is possible to see that all approaches GRES3D have scores that are numerically close to each other, which means that quality and type of sensor is no longer a determinant factor to achieve good results in road detection task using $3 \mathrm{D}$ points. Figure 5.2 

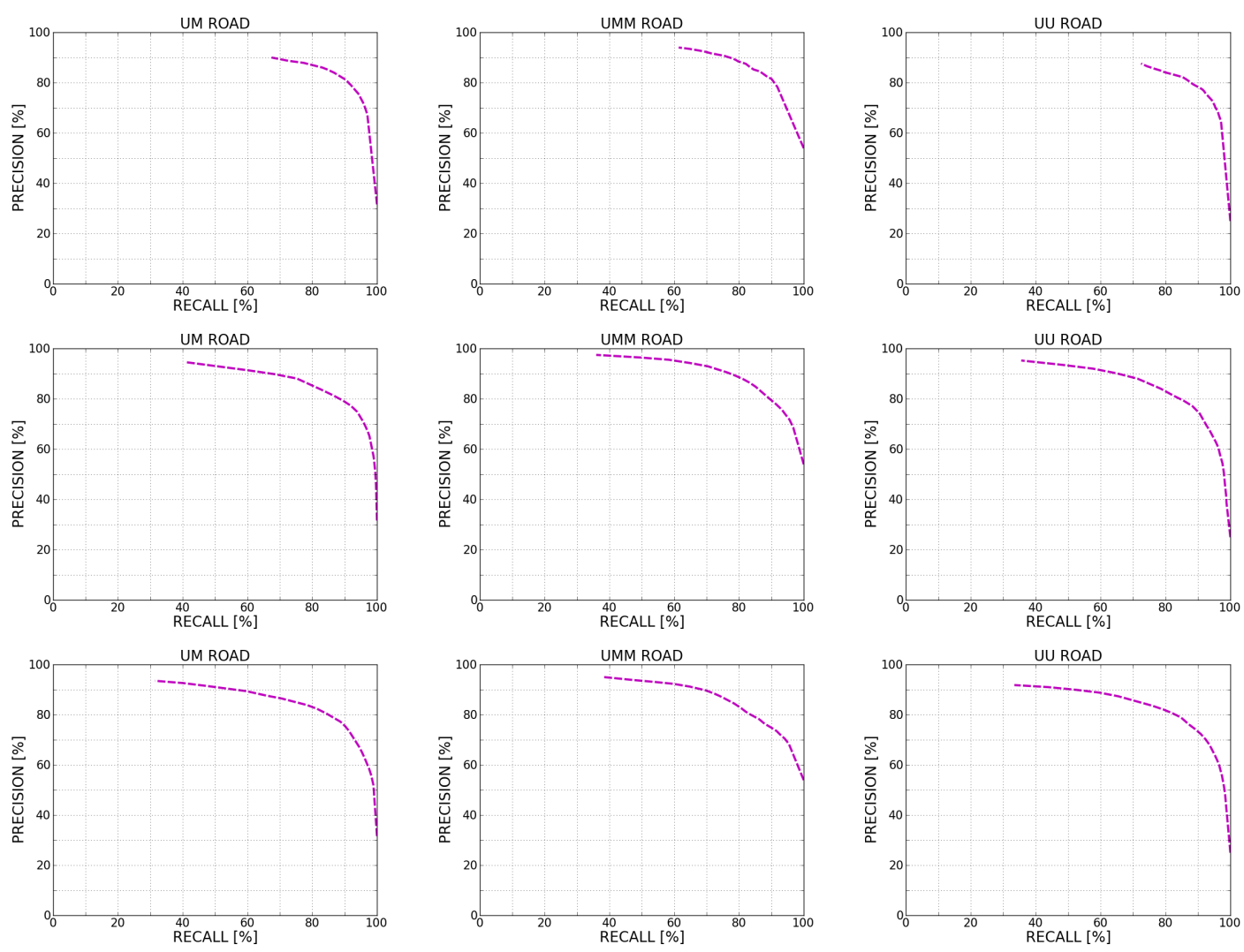

Figure 5.2: The results in the first row belongs to GRES3D+VELO, while second contains results from GRES3D+SELAS and finally, the third row shows GRES3D+SGBM. A comparison between GRES3D+VELO and stereo approaches shows that 3D-LIDAR system has few threshold values that changes the recall range which means a more stable approach. Graphics available in KITTI website.

shows precision-recall graphics for all three categories for all approaches GRES3D, and helps to concludes that GRES3D+VELO has few threshold values that changes the recall range (if compared with stereo approaches) which means a little variation in the truly relevant results even ranging the precision value.

A visual comparison between our approaches are shown in Figure 5.3 for UM category, Figure 5.4 for UMM, and Figure 5.5 for UU category, by default, the first row is result from GRES3D+VELO followed by GRES3D+SELAS in the second row and GRES3D+SGBM in the third row. The Figure 5.3 shows false negative area (red area) in the second row (GRES3D+SELAS) due a noise in disparity map caused by the road marking whose was classified as obstacle in the image. Furthermore, is possible to see a pattern of false negative on left side of all three images which is caused by chosen precision-degree of polar-range-histogram. The Figure 5.4 shows a false negative (red area) on right side in the second row which is caused by the imprecision 
of Modified ELAS. The remaining false negative on the left side of all three images are caused by the fact that the proposed algorithm does not detect the opposite side road (a discussion about this problem is provided in section 5.2). Since we do not use previous information, the rail area, i.e. the false positives (blue areas) occurs because, even with 3D-LIDAR sensor, our obstacle detection method was not able to detect these small curbs after a range of more less $15 \mathrm{~m}$. In Figure 5.5, the red area is caused by noise from 3D-LIDAR and from stereo matching algorithms. The blue area is caused by the inaccurate disparities in the near range.

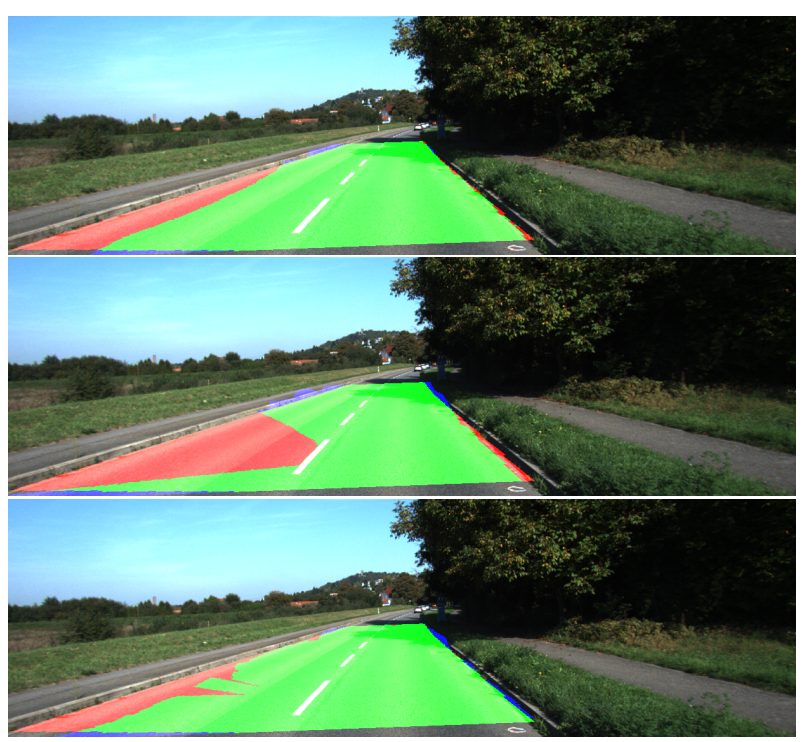

First row belongs to GRES3D+VELO, second row to GRES3D+SELAS and third row to GRES3D+SGBM. It shows false negative area (red area) in the second row due a noise in disparity map caused by the road marking whose was classified as obstacle in the image. Furthermore, is possible to see a pattern of false negative on left side of all three images which is caused by chosen precision-degree of polar-range-histogram.

Figure 5.3: Image results from all three approaches for the Urban Marked category.

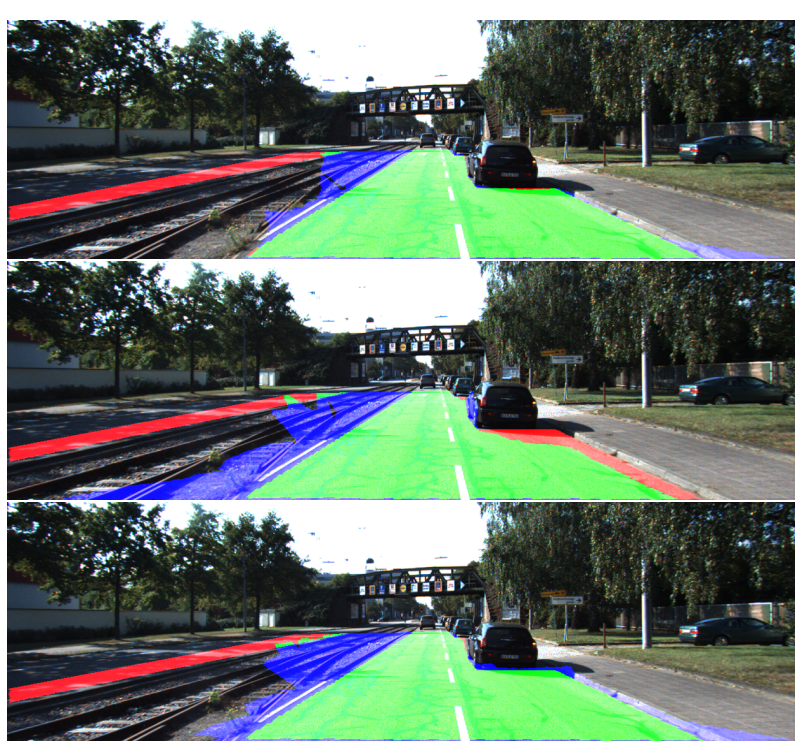

First row belongs to GRES3D+VELO, second row to GRES3D+SELAS and third row to GRES3D+SGBM. The false negative (red area) on right side in the second row is caused by the imprecision of SELAS. The left false negative on the left side of all three images are caused by the fact that the proposed algorithm does not detect the opposite side road. The rail area, i.e. the false positives (blue areas) occurs because our obstacle detection method was not able to detect these small curbs in this range (more less $15 \mathrm{~m}$ ).

Figure 5.4: Image results from all three approaches for the Urban Multiple Marked category. 


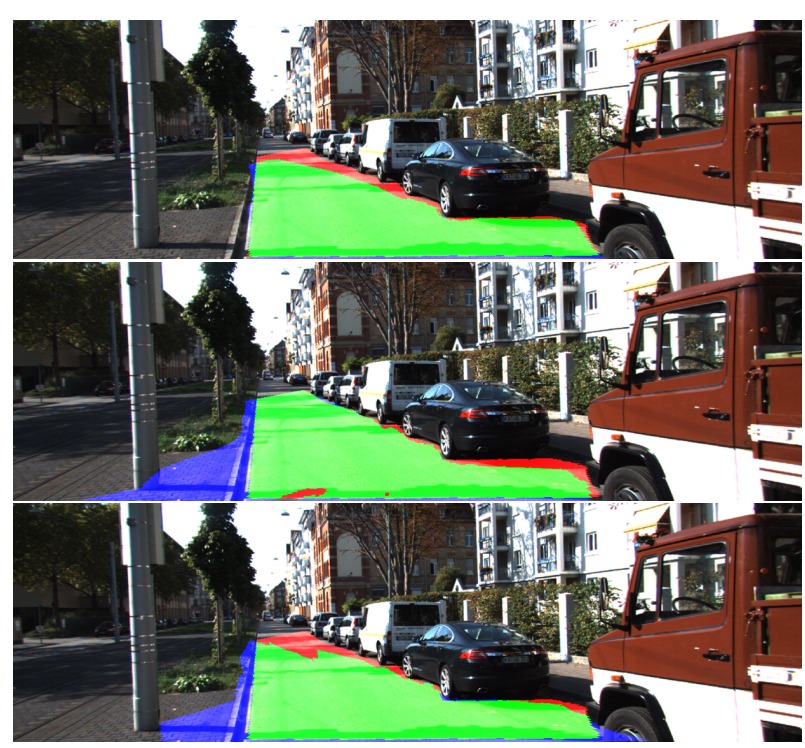

First row belongs to GRES3D+VELO, second row to GRES3D+SELAS and third row to GRES3D+SGBM. The red area is caused by noise from 3D-LIDAR and from stereo matching algorithms. The blue area is caused the inaccurate disparities in the near range.

Figure 5.5: Image results from all three approaches for the Urban Unmarked category.

Based on runtime showed in Table 5.2, we can state that our system takes approximately 55 milliseconds to detect obstacles and estimate the road, since the 3D-LIDAR approach runs only in 60 milliseconds and the stereo-camera approach with ELAS runs in approximately 110 milliseconds ( 55 milliseconds is the time it takes the disparity algorithm to process the relevant points from an image). Since it uses only local information and 3D data in a list of points, it can be parallelizable to decrease the runtime. Furthermore, it can also be combined with other approaches that use other types of information, like texture to improve the evaluation scores.

\subsection{Ground-Truth Images From training-dataset}

We consider that the road estimation problem should be handled as a local context problem, therefore only the ego-road should be detected. If a ground-truth image does not comply with this condition (see example in Figure 5.6), this ground-truth will degrade the performance of any ego-road estimation method. Since this type of system will never detect the opposite road in Figure 5.6, the false negative rate (FNR) will be increased, and consequently, the recall and other metric values will decrease.

In order to show the performance degradation due the evaluation using ground-truth images from KITTI-ROAD dataset as showed in Figure 5.6. The road estimation method was evaluated separately from obstacle estimation method using a pseudo-optimal obstacle detection result as input to estimate the road area. In other words, using the ground-truth images from training-dataset, the obstacle-confidence of a node being an obstacle is zero if it is inside the 


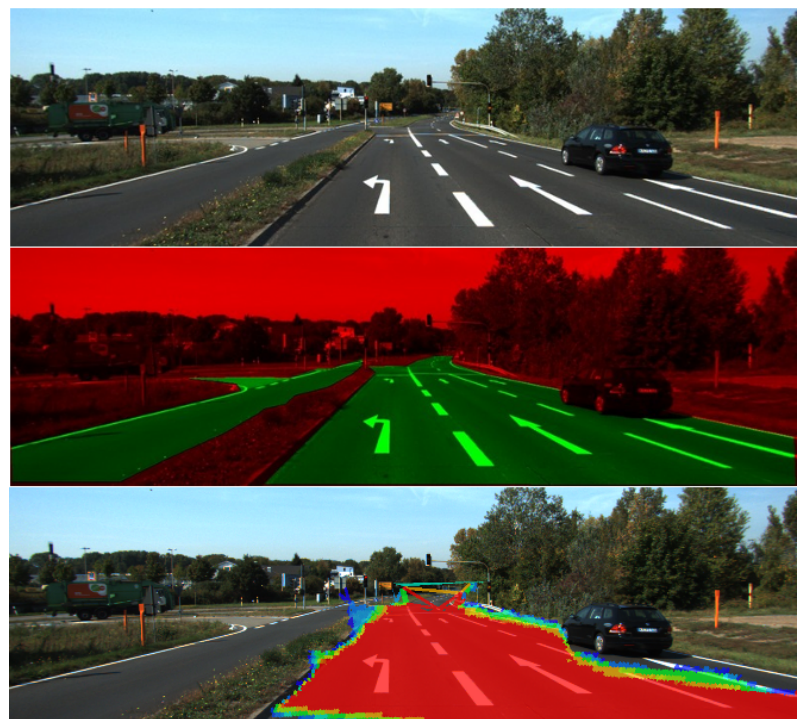

Figure 5.6: Example of ground-truth image that will degrade the performance of any ego-road estimation method, since it is not able to detect the side road.

Upper Image: Original Image. Middle Image: Ground-truth from Kitti benchmark; green pixels represent the expected road area and red pixels represent no-road area.

Bottom Image: Output from our method using a sensors system composed by a 3D-LIDAR and Monocular Camera.

road area (green-area of middle image from Figure 5.6) defined by KITTI and is 1.0 if it is not, i.e. 1.0 if the point is inside to red area (the Figure 5.7 depicts this process). Table 5.4 shows the results from our road estimation method by changing the precision-degree used to define the number of bins of the polar-range-histogram. Since there is no noise in this pseudo-optimal obstacle-detection, all previously chosen parameters were used in this evaluation except by the number of possible $\beta$ 's, and its value equals to 0.5 . We can consider the scores from Table 5.4 as the maximum possible hit rate that our road estimation method can achieve using any type of sensor with the respective precision-degree.
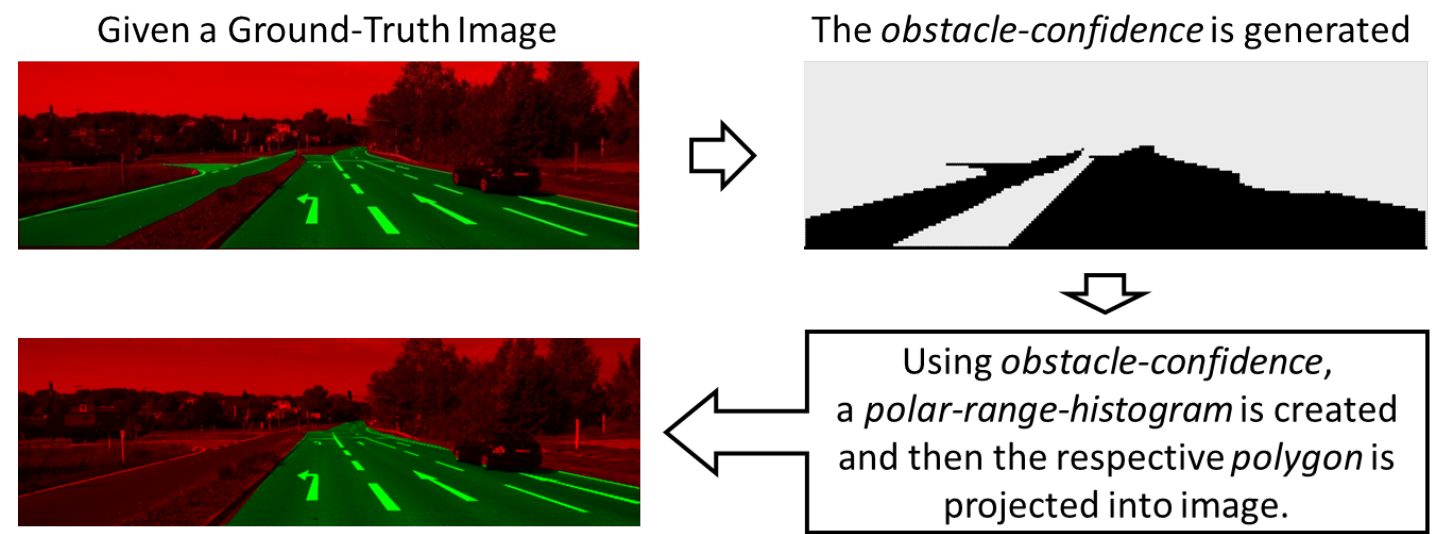

Figure 5.7: Given a ground-truth image (upper left image), the obstacle estimation method creates an obstacle-confidence map (upper right image) where lighter color represents more certainty about the pixel be an obstacle and darker pixels represents more certainty about the pixels be road. After that, the road estimation method generates the polygon that represents the road area, i.e. the green area from (bottom left image). 
Table 5.4: Comparison of several precision-degree in road-estimation evaluation [\%] using the ground-truth images as estimated-obstacles. The results shows that is not possible to achieve $100 \%$ of MaxF due to only ego-road be detected.

\begin{tabular}{c|c|c|c|c|c|c} 
precision-degree & MaxF & AP & PRE & REC & FPR & FNR \\
\hline $1^{\circ}$ & 94.07 & 88.89 & 92.53 & 95.70 & 4.44 & 4.30 \\
$2^{\circ}$ & 94.63 & 91.57 & 95.15 & 94.12 & 2.99 & 5.88 \\
$3^{\circ}$ & 94.29 & 92.21 & 93.85 & 94.75 & 3.66 & 5.25 \\
$4^{\circ}$ & 93.76 & 92.26 & 93.13 & 94.40 & 4.23 & 5.60 \\
$5^{\circ}$ & 93.25 & 92.20 & 93.54 & 92.96 & 3.99 & 7.04 \\
$6^{\circ}$ & 92.62 & 92.08 & 93.71 & 91.56 & 3.87 & 8.44 \\
$7^{\circ}$ & 92.08 & 90.69 & 92.51 & 91.69 & 4.44 & 8.31 \\
$8^{\circ}$ & 91.67 & 90.56 & 91.56 & 91.85 & 4.75 & 8.15 \\
$9^{\circ}$ & 91.27 & 90.34 & 91.51 & 91.10 & 4.74 & 8.90 \\
$10^{\circ}$ & 90.74 & 90.18 & 91.14 & 90.41 & 4.96 & 9.59 \\
$11^{\circ}$ & 90.49 & 90.03 & 91.40 & 89.66 & 4.74 & 10.34 \\
$12^{\circ}$ & 90.07 & 89.81 & 91.08 & 89.15 & 4.91 & 10.85 \\
$13^{\circ}$ & 89.83 & 87.85 & 91.31 & 88.48 & 4.72 & 11.52 \\
$14^{\circ}$ & 89.63 & 87.75 & 91.34 & 88.05 & 4.71 & 11.95 \\
$15^{\circ}$ & 89.11 & 87.45 & 90.71 & 87.65 & 5.08 & 12.35 \\
$16^{\circ}$ & 89.01 & 87.33 & 91.27 & 86.95 & 4.68 & 13.05 \\
$17^{\circ}$ & 88.63 & 85.23 & 91.07 & 86.40 & 4.82 & 13.60 \\
$18^{\circ}$ & 88.15 & 85.10 & 90.17 & 86.31 & 5.33 & 13.69 \\
$19^{\circ}$ & 88.28 & 85.49 & 91.16 & 85.66 & 4.67 & 14.34 \\
$20^{\circ}$ & 87.61 & 84.74 & 89.99 & 85.46 & 5.28 & 14.54
\end{tabular}

Based on Table 5.4, since the best achieved MaxF is $94.63 \%$ from the instance using precision-degree $=2^{\circ}$, it is possible to conclude that the current ego-road detection system can not achieve 100\% of MaxF. In addition, the differences of MaxF and AP between these instances with different precision-degree show a nearly small linear variation between the results (see graphic in Figure 5.8). In order to build a system with integer number of bins, and since the maximum possible results are numerically close, the precision-degree $=5^{\circ}$ was chosen to this experiment.

\subsection{D LIDAR + Monocular Image Sensor Approach}

In this section we present the quantitative results of our experiments using the 3D-LIDAR points from KITTI-ROAD training-dataset. Table 5.5 shows the same type of evaluation scores described by (Fritsch et al., 2013). This evaluation shows that results from GRES3D+VELO has a relevant (more less 10\%) difference from the maximum possible value showed in Table 5.4(Line 


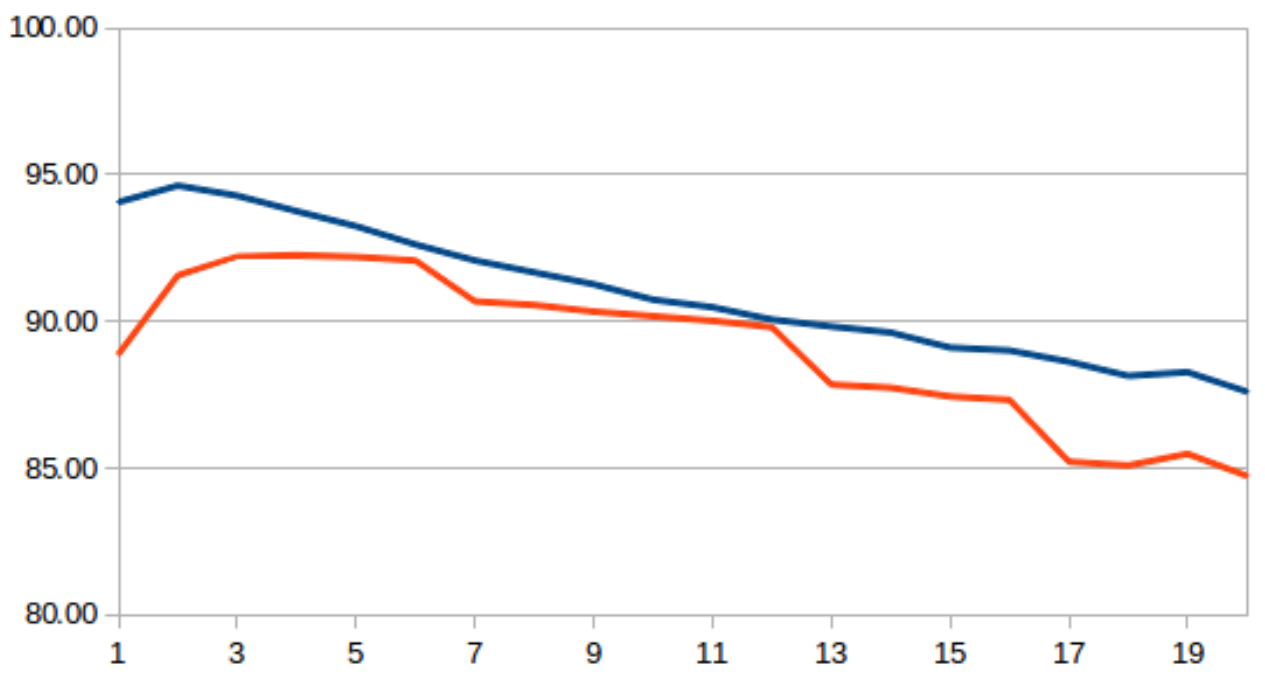

Figure 5.8: The MaxF and AP values (\% scores in y-axis) between these instances with different precision-degree's ( $x$-axis) show a nearly small linear variation between the results. Blue line represents MaxF and orange line represents AP.

5). Taking into account the runtime of 60 milliseconds, our method achieved satisfactory confidence degrees with a high confidence level, as shown in Figure 5.9. However, as one of the limitations of our method is the assumption that the road is delimited by curbs, when this assumption is not true, our system fails to detect only road area by incorporating wrong areas as sidewalks (Fig. 5.9a) or opposite roads (Fig. 5.9b). Despite the high accuracy of the 3D points of the 3D-LIDAR sensor, our method still fails to detect curbs in a far range (Fig. 5.9c) due the euclidean distance between the points be too large for the chosen constant $\theta$. The authors believes, that the incorporation of a function instead of this constant and other visual features can improve the method without severely compromising the current processing time.

Table 5.5: Road-area evaluation [\%] of (GRES3D+VELO) in a Training Dataset.

\begin{tabular}{c|c|c|c|c|c|c} 
Benchmark & MaxF & AP & PRE & REC & FPR & FNR \\
\hline UM_ROAD & $84.40 \%$ & $83.97 \%$ & $81.37 \%$ & $87.65 \%$ & $8.54 \%$ & $12.35 \%$ \\
UMM_ROAD & $87.52 \%$ & $89.40 \%$ & $86.61 \%$ & $88.45 \%$ & $14.18 \%$ & $11.55 \%$ \\
UU_ROAD & $83.20 \%$ & $79.44 \%$ & $76.96 \%$ & $90.54 \%$ & $9.28 \%$ & $9.46 \%$ \\
\hline URBAN_ROAD & $85.04 \%$ & $84.27 \%$ & $81.65 \%$ & $88.88 \%$ & $10.66 \%$ & $11.12 \%$
\end{tabular}

Despite the satisfactory scores showed in Table 5.5 is important to notice that all score values are computed over all pixels from all images from training-dataset after selection of threshold that maximizes F1-measure over all dataset. This type of evaluation provides insights about the general performance of a classifier and it is fair to compare several classification methods but it is not reliable to determine whether a particular classifier is good enough to be used in 

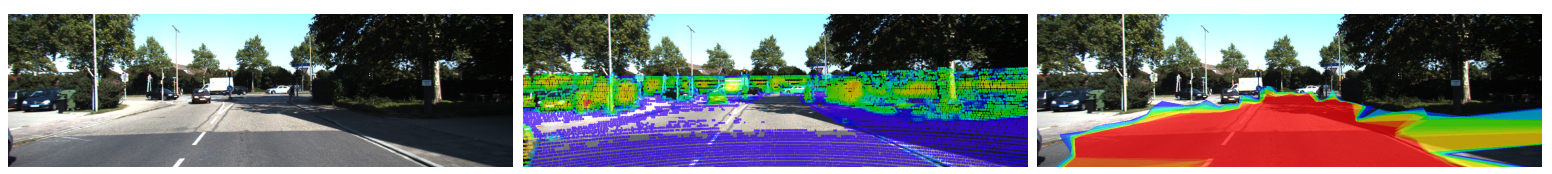

(a) 3D-LIDAR result fails by incorporating wrong area of sidewalk on left.
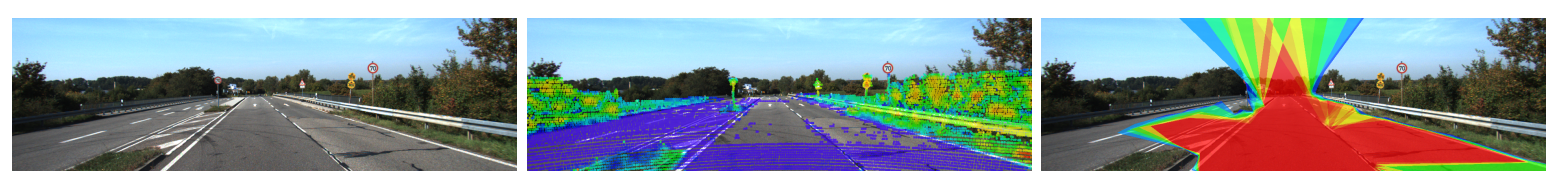

(b) 3D-LIDAR result fails by incorporating wrong area of opposite road on right.
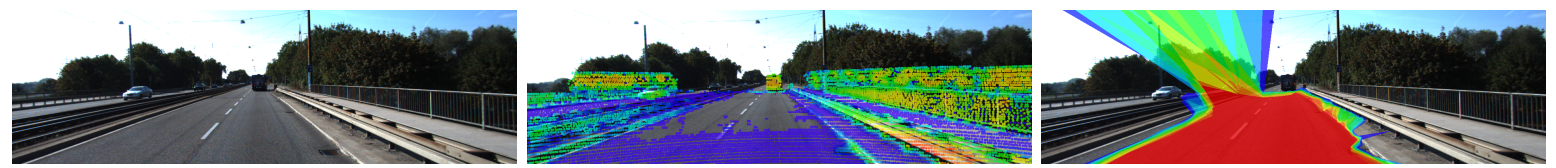

(c) 3D-LIDAR result with a high-confidence degree but without detection of curbs in a far range.

Figure 5.9: Output from our proposed method using 3D-LIDAR sensor. From left to right, the first figure shows the original image, the middle shows the obstacle-confidence using 3D-LIDAR points, and last the third is the road-confidence in perspective view. All confidence-maps are shown in thermal-color style images, i.e. red color means the highest value while blue the lowest non-zero value, note that zero confidence means the original color (RGB).

a large scenario such as a city. For an intelligent vehicle application, which aims to be robust to several scenarios over many conditions, the author of this thesis believes in the addition of an evaluation based on a graphical representation known as boxplot (Tukey, 1977), to provide insights about the accuracy of an classifier over every single frame provided in a dataset. Therefore, Figure 5.10 shows three sets of boxplots where each set shows four metric evaluations (Precision, Recall, FPR, FNR), i.e. one set for each category from training-dataset. The main idea of this evaluation is detect if a classifier has disadvantages in a specific scenario/condition, i.e. detect if the classifier has good performance in scenario $\mathrm{A}$ and an unacceptable performance over scenario B, where in this case, each scenario is just a single frame.

Figure 5.10 shows, respectively, the variation (Boxplot information) of (Precision, Recall, FPR, FNR) in a group in which each element is a metric computed for each image. Given the property of the Recall metric, let's assume without loss of generality, that a Recall value larger or equal to $80 \%$ in a single image is an acceptable result. Given the characteristics of boxplot that divides a group in four parts separated by first quartile, median and third quartile, we can state that GRES3D+VELO is able to achieve acceptable results in more than $75 \%$ of all tested images in all three categories. Furthermore, we can highlight some outliers(or unacceptable results) with very poor results by the symbol ' + ' in red, one of them is showed in Figure 5.11, 

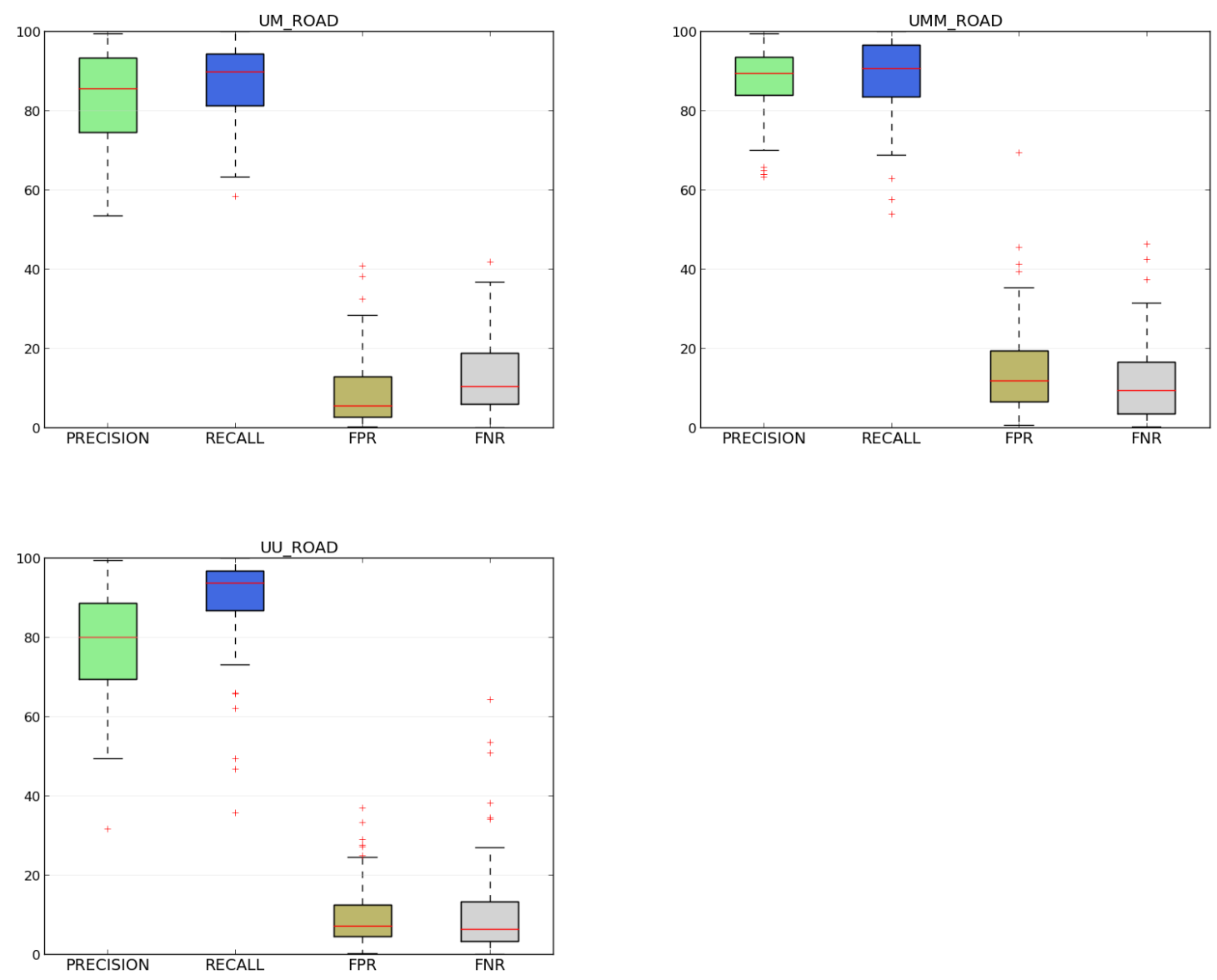

Figure 5.10: Single-Frame Evaluation of GRES3D+VELO in Boxplot. It shows three sets of boxplots where each set shows four metric evaluations (Precision, Recall, FPR, FNR) for each category from training-dataset.

and we can state that has poor results because is a scenario where road is not delimited by any obstacle or curb. Based on this sample, the author of this thesis shows that is possible to track and try to solve any possible scenario available in a dataset by the use of this metric representation. Furthermore, conclusions about the performance of a specific classifier over an big scenario like an entire city, can be geo-referenced and used to make a more fair comparison between several classifiers.

\subsection{Stereo Image Sensor Approach with Sparse-ELAS}

In this section we present the quantitative results of our experiments using 3D points generated after perform a modified version of a stereo correspondence algorithm known as ELAS. 

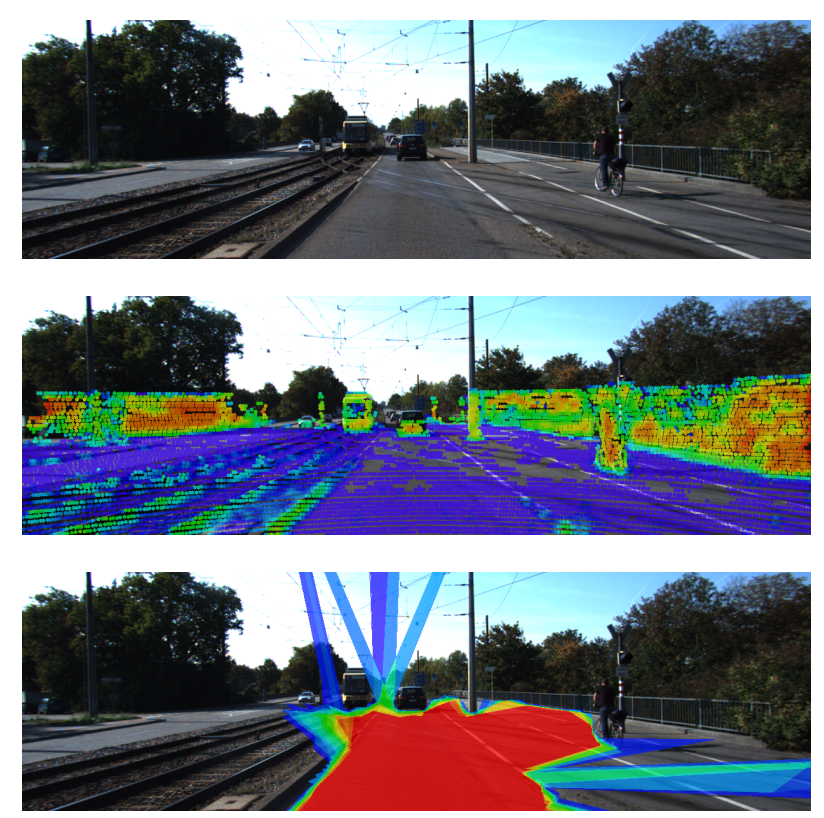

Figure 5.11: Worst result detected by the Boxplot representation. This image was highlighted as red ' + ' in category Urban Unmarked from the graphics displayed in Figure 5.10. This failure is caused by the non-existence of curbs or obstacles to delimit this road.

In this work, the 3D point is composed by coordinates $(u, v, d)$ and all pair of images are available in KITTI-ROAD training-dataset in order to have scores that allows us a way to compare all approaches presented in this thesis. As mentioned before, the difference between GRES3D+SELAS and GRES3D+VELO are only three parameters, $\theta, \lambda$ and roi. Table 5.6 shows the same type of values as Table 5.5 and at first sight is possible to see that the numerical values are very close (difference of $0.54 \%$ in URBAN line) between these approaches. This closeness allow us to believe that systems with a stereoscopic camera sensor can achieve similar results to approaches that use only 3D-LIDAR sensor. Since the processing time of this approach is 50 milliseconds slower than GRES3D+VELO, and the current state of art of stereo correspondence algorithms take more time, or dedicated hardware to process the disparity map, the results showed in Table 5.6 can be considered satisfactory. The boxplot graphics are shown in Figure 5.12.

The boxplot information shown in Figure 5.12 shows the same conclusion of GRES3D+VELO, i.e. that $75 \%$ of all tested images has acceptable results. However, recall value of remaining images (25\%) are spread over a larger range between $85 \%$ and $20 \%$, and the showed outliers achieves worst numerical results. Figure 5.13 shows the worst result highlighted as red ' + ' in category Urban Unmarked. This failure is caused by the low confidence in the road area caused 
Table 5.6: Road-area evaluation [\%] of (GRES3D+SELAS) in a Training Dataset.

\begin{tabular}{c|c|c|c|c|c|c} 
Benchmark & MaxF & AP & PRE & REC & FPR & FNR \\
\hline UM_ROAD & $84.91 \%$ & $85.56 \%$ & $82.94 \%$ & $86.97 \%$ & $7.61 \%$ & $13.03 \%$ \\
UMM_ROAD & $86.27 \%$ & $90.78 \%$ & $85.81 \%$ & $86.73 \%$ & $14.87 \%$ & $13.27 \%$ \\
UU_ROAD & $82.57 \%$ & $83.20 \%$ & $76.60 \%$ & $89.55 \%$ & $9.36 \%$ & $10.45 \%$ \\
\hline URBAN_ROAD & $84.58 \%$ & $86.51 \%$ & $81.78 \%$ & $87.75 \%$ & $10.61 \%$ & $12.25 \%$
\end{tabular}
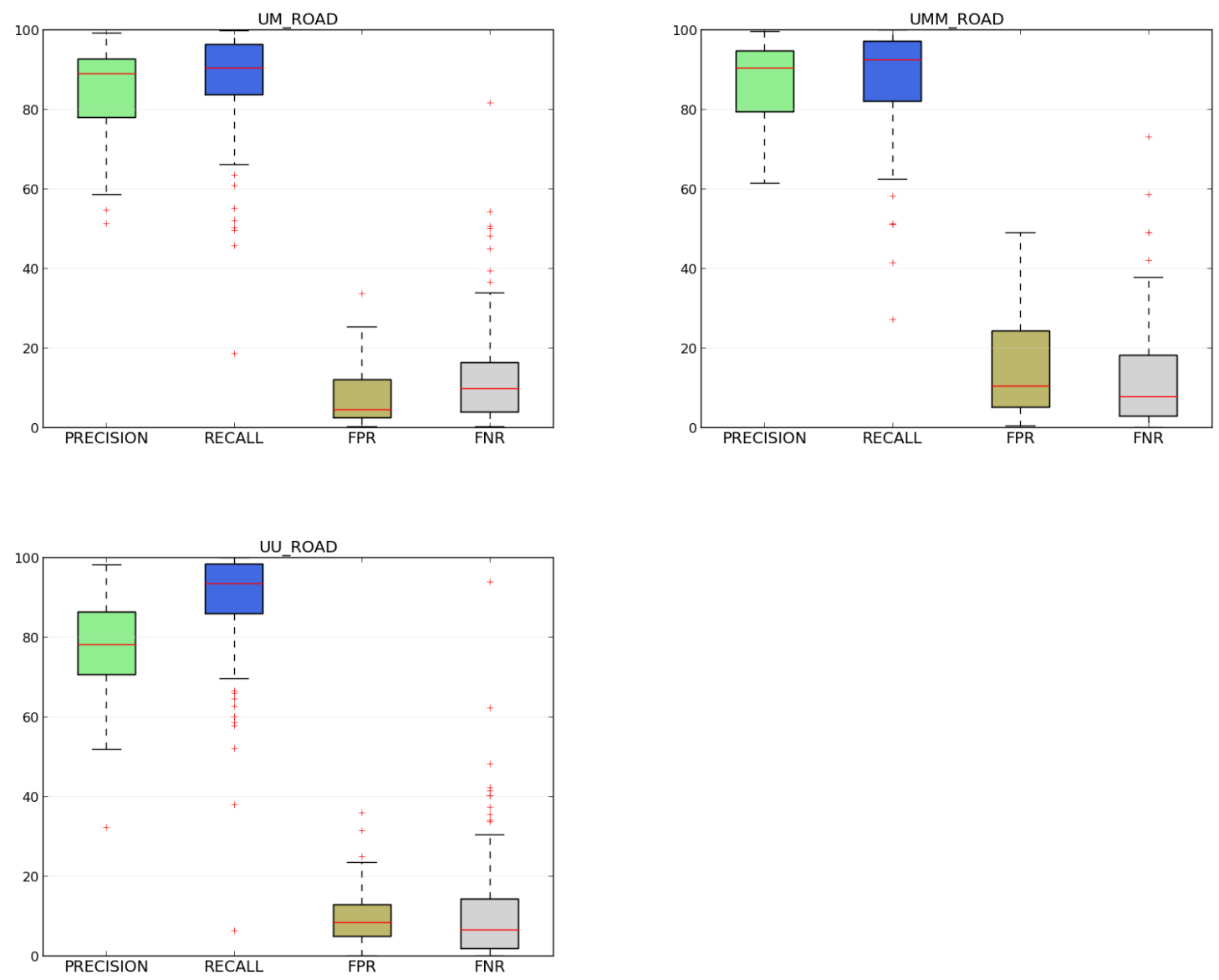

Figure 5.12: Single-Frame Evaluation of GRES3D+SELAS in Boxplot. It shows three sets of boxplots where each set shows four metric evaluations (Precision, Recall, FPR, FNR) for each category from training-dataset.

by noise in the disparity map. In other words, after the selection of best-threshold which maximizes F1-measure, a huge amount of pixels (green and blue pixels) are classified as non-road. Despite the poor results generated by low confidence, the reader can see a consistently classification result, i.e. even with low recall value, our system still shows a good performance in the road detection task. This case of evaluation allow us to believe that poor results are derived, 
most of times, due the needs to compare with binary classification systems that selects a threshold value to binarize the output of a estimation method. Furthermore, the author of this thesis believes that temporal information (accumulation of frames) can improve the confidence degree and then avoid these low scores.
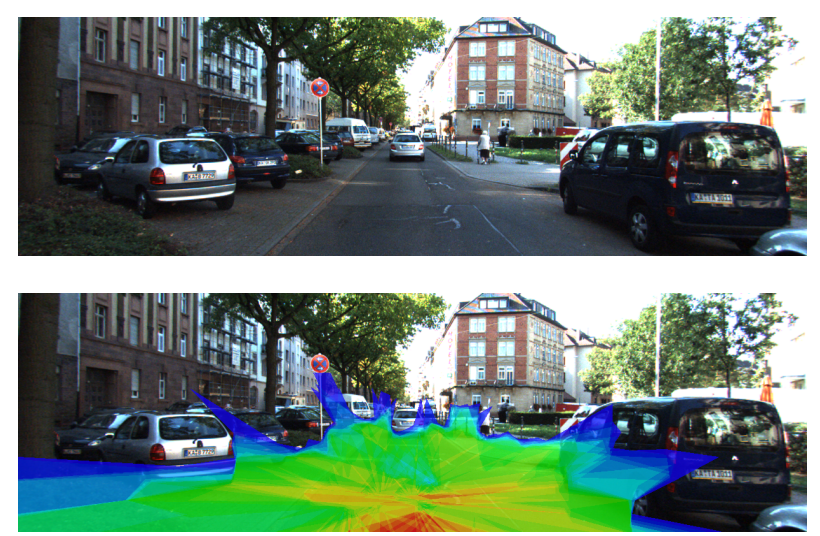

Figure 5.13: Worst result detected by the Boxplot representation. This image was highlighted as red ' + ' in category Urban Unmarked from the graphics displayed in Figure 5.12. This failure is caused by noise in the disparity map which implies in a low confidence degree in the road area. After the selection of best-threshold which maximizes F1-measure, a huge amount of pixels (blue pixels) are classified as non-road. Despite the poor results generated by low confidence, you can see a result consistently classified.

Other results are shown in Figure 5.14, these images have high road-confidence in all three categories and helps to show the good performance of the proposed method. Some images shows robustness in shadow situations, noise caused by road markings and failures in asphalt, and road area limited by obstacles like cars and walls. Furthermore, it is possible to see that sometimes the sidewalk is included in the road area but always with low confidence (blue and green colors). As previously mentioned, the use of temporal information and statistical methods can improve the performance of the proposed method. 

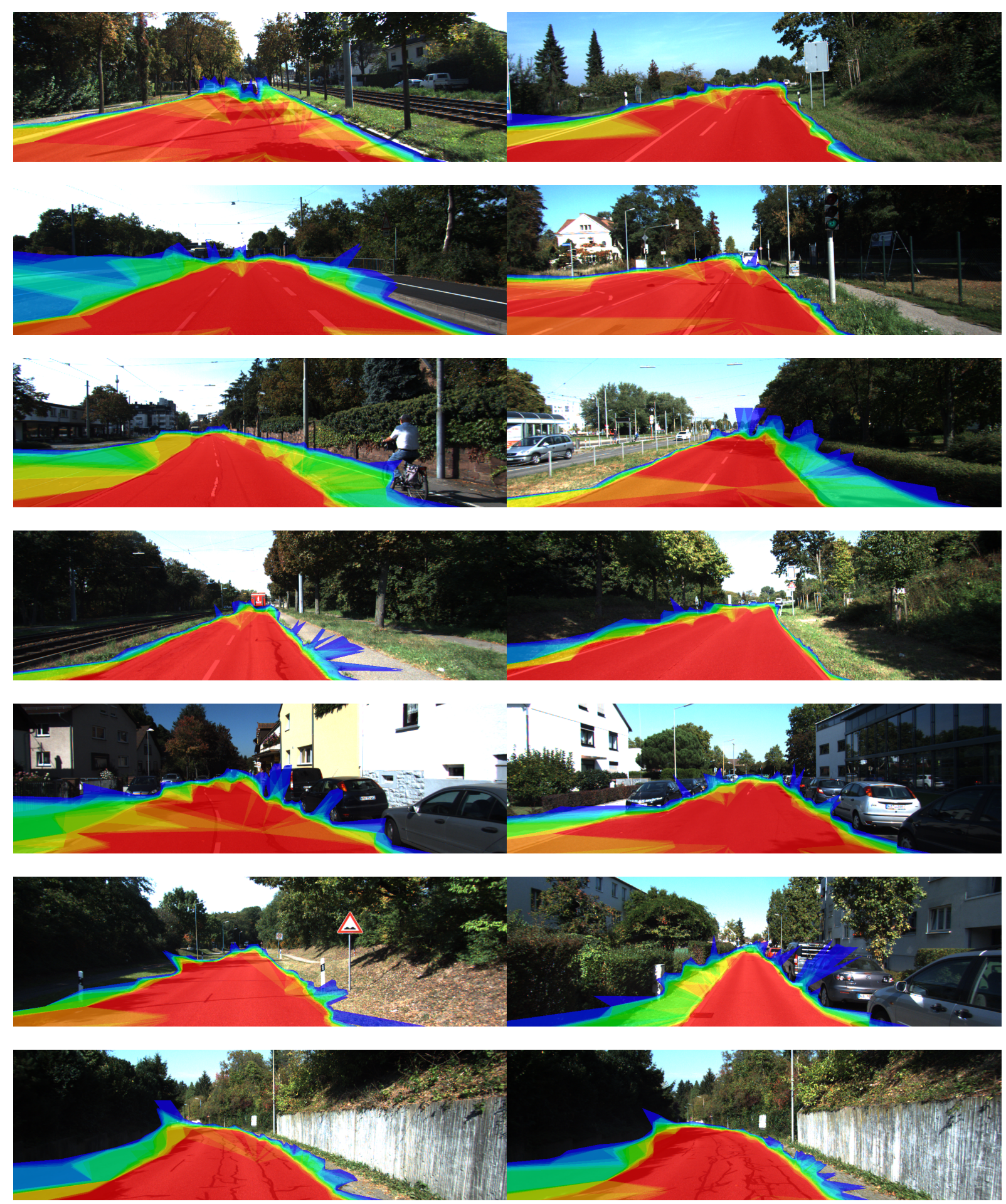

Figure 5.14: Good results of classification generated by GRES3D+SELAS showed in thermal color style, i.e. red means highest confidence degree about pixel be road and blue means lowest road-confidence that are greater than zero. 


\subsection{Stereo Image Sensor Approach with Semi-Global Block-Matching}

In order to show the absence of dependence of stereo correspondence algorithm, this thesis also evaluate the proposed method using a very common method used by research community, the Semi-Global Block Matching (SGBM) (Hirschmueller, 2008) available in OpenCV Library. All scores are shown in Table 5.7 and despite being the worst result obtained by our method, it is fair to highlight that is only $3 \%$ less than GRES3D+SELAS in the last line of MaxF column.

Table 5.7: Road-area evaluation [\%] of (GRES3D+SGBM) in a Training Dataset.

\begin{tabular}{c|c|c|c|c|c|c} 
Benchmark & MaxF & AP & PRE & REC & FPR & FNR \\
\hline UM_ROAD & $81.08 \%$ & $84.02 \%$ & $81.36 \%$ & $80.81 \%$ & $7.88 \%$ & $19.19 \%$ \\
UMM_ROAD & $83.30 \%$ & $89.15 \%$ & $80.65 \%$ & $86.14 \%$ & $21.43 \%$ & $13.86 \%$ \\
UU_ROAD & $80.76 \%$ & $81.82 \%$ & $78.62 \%$ & $83.03 \%$ & $7.73 \%$ & $16.97 \%$ \\
\hline URBAN_ROAD & $81.71 \%$ & $85.00 \%$ & $80.21 \%$ & $83.33 \%$ & $12.35 \%$ & $16.67 \%$
\end{tabular}

The main differences between these approaches are more accuracy in near range (approximately first $15 \mathrm{~m}$ ) and frequent lack of valid disparity values in some regions of an image in disparity map generated by SGBM. These differences allows better detection of curbs and obstacles in near range for GRES3D+SGBM but inaccuracy in medium and far range (see Figure 5.15). Another factor that decreases the performance of this approach is that SGBM is less robust to shadow situations and edges of road markings. Some post-processing filters could be employed to improve performance, however the overall system would be slower. Since our system with SGBM algorithm has 0.72 seconds of processing time because SGBM is much slower than modified version of ELAS (650 ms to generate disparity map) SGBM is not a satisfactory approach for real time applications with no hardware improvements (it is not a determinant factor since it can be replaced by optimized versions like implementations in GPU or a dedicated hardware).

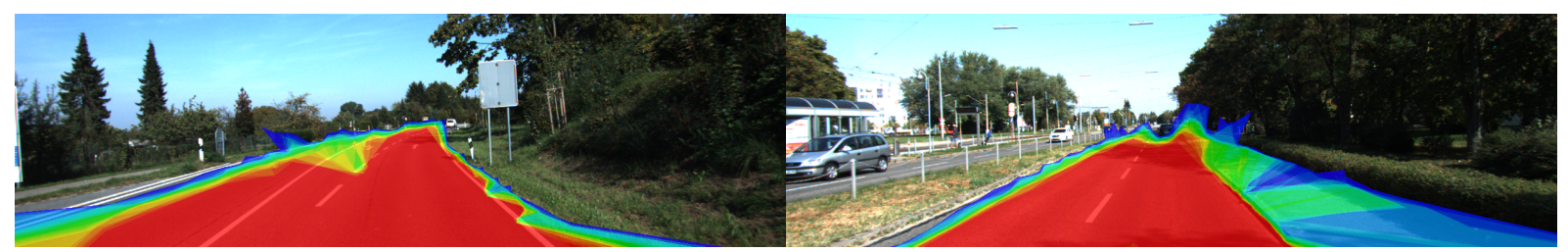

Figure 5.15: Samples of results from GRES3D+SGBM. It is possible see good detection in near range and failure in road markings edges. 
The evaluation based on the boxplot information shows a slightly variation in comparison with GRES3D+SELAS. However it is possible see that GRES3D+SGBM has less images where recall value does not achieve at least $50 \%$, which means that SGBM has a more homogeneous result even than be slightly worse.
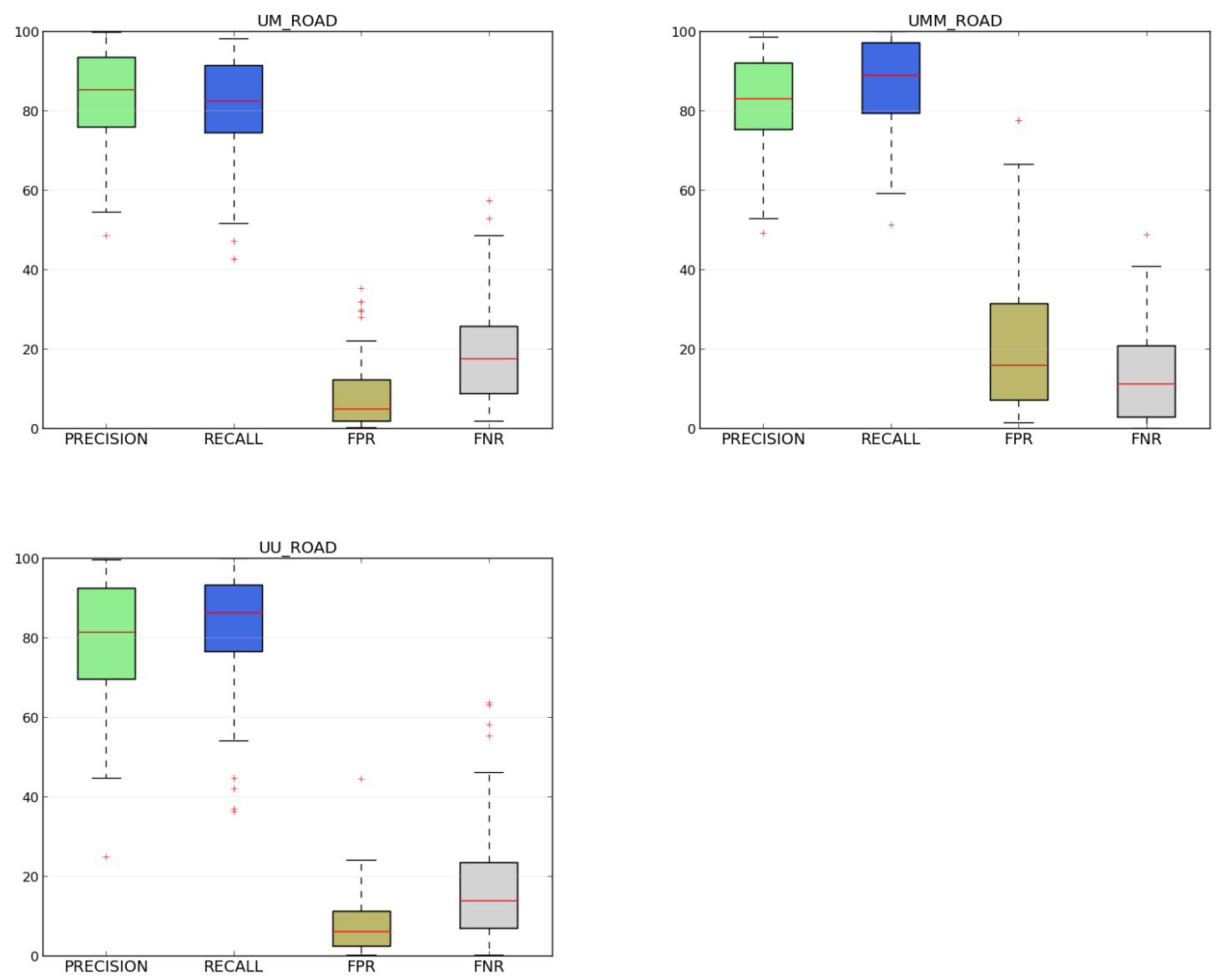

Figure 5.16: Single-Frame Evaluation of GRES3D+SGBM in Boxplot. It shows three sets of boxplots where each set shows four metric evaluations (Precision, Recall, FPR, FNR) for each category from training-dataset.

\subsection{Considerations}

This chapter presented several evaluation results from the proposed road estimation method. One of them is an online evaluation available in KITTI-ROAD Benchmark, which allow us to compare our method with several other approaches developed by researchers over the world and to demonstrate that our method belongs to top ten group and it is one of the fastest approaches. 
Furthermore, this chapter shows an evaluation over the ground truth data that allow us to check that our approach never will achieve $100 \%$ in all scores, since our method can only detect the ego-road. The tree remaining sections of this chapter present an evaluation more detailed of each sensor setup and using an visualization approach known as Boxplot our method was evaluated using each frame as an single scenario. This visualization allow us to detect and verify that the worst result is only a bad result because it does not have enough confidence about the road area. 


$\frac{1}{6}$

\section{Other Contributions and Applications}

This chapter reports on some contributions made in cooperation with other students. The first is a road marking enhancement method used in gray-scale images, whose the main objective is to highlight only the road markings and handle situations of shadows without any image transformation. The second is a Lane Departure Warning System developed to cooperate with a monocular camera fixed in CaRINA 2 (Batista et al., 2014). The third is an automatic algorithm to map urban road networks. This system aims at automatically detecting intersections and lanes to integrate semantic and topological information into a more sophisticated map structure (Zoccoler et al., 2014). The last contribution is a public demonstration of CaRINA-2 autonomous navigation using a modified version of our obstacle detector method for simplifying the adaptive cruise control (ACC). Such a demonstration has drawn plenty of attention and considerable coverage by the media.

\subsection{Road Markings Enhancement}

Road markings detection is an important issue for autonomous vehicles and ADAS, as Lane Departure Warning and Lane Keeping. Although progress has been made in recent years, the literature has shown several approaches with satisfactory results only for highway-like scenarios and restricted situations. Most of them assume the vehicle is initially set to the same direction of the target lane. This section presents a road marking enhancement method to be used by 
ADAS and Mapping systems. It can be applied to both bird-eye-view and perspective images used by algorithms of lane estimation.

The original image is defined as $\mathbf{I}(m, n)$, where $m$ is the number of lines and $n$ is the number of columns of an image. The main idea is to accumulate positive differences between a target pixel $\mathbf{I}(u, v)$ and its neighbours in several orientations. Given a set of orientations $\Phi$, a marking-confidence is computed through the accumulation of the smaller difference between the left and right pixels for each distance chosen in each orientation $d$. In other words, for each pixel, a new value $\mathbf{M}(u, v)$ is calculated based on the sum of all smaller differences from all pixels located in several different orientations and distances, as shown in Equation 6.1:

$$
\mathbf{M}(u, v)=\sum_{d \in \Phi} \sum_{i=1}^{S} \min \left(\begin{array}{c}
\max \left(\mathbf{I}(u, v)-l_{d}^{i}, 0\right), \\
\max \left(\mathbf{I}(u, v)-r_{d}^{i}, 0\right)
\end{array}\right)
$$

where $\Phi$ is a set of orientations, $S$ is a constant arbitrarily defined that represents the furthest neighbouring pixel, $\mathbf{I}(u, v)$ is the gray-scale value from the target pixel, $l_{d}^{i}$, and $r_{d}^{i}$ are, respectively, the gray-scale value from $i^{t h}$-pixel on the left and right sides of $\mathbf{I}(u, v)$ in orientation $d$, as shown in Fig. 6.1.

Figure 6.1: Each red box represents a pixel. The method calculates the difference of the value of the pixels in the same orientation (represented by the black lines) and distance from the target pixel (central box). Several orientations and distances are used for handling noise.

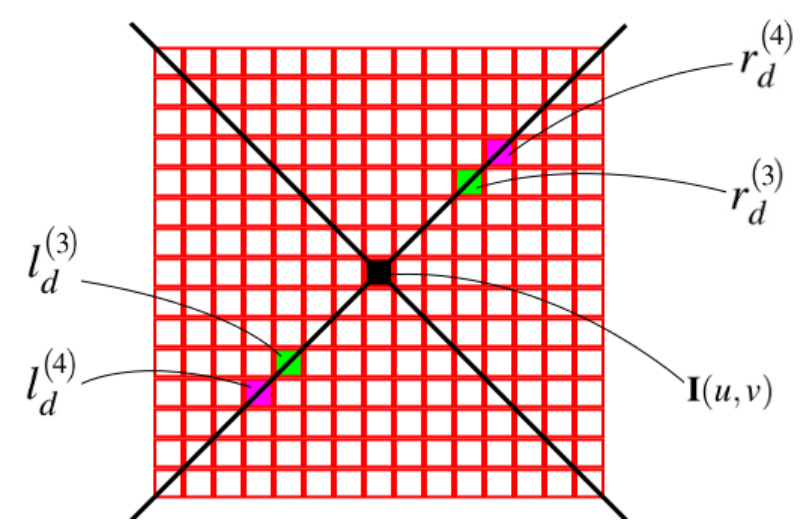

The results are shown in Figure 6.2. Even in situations of shadows, the enhancement method highlighted all road markings. Since neither a specific orientation, nor the width of the marking are assumed, arrows and other traffic signals are enhanced with no tuning in the internal parameters, i.e. no changes $\Phi$ and $S$ are made.

\subsection{Lane Departure Warning}

Unlike other approaches in the field, this work proposed detection of lane and localization of vehicle on it without the requirement of calibration. The detection system is composed by several 

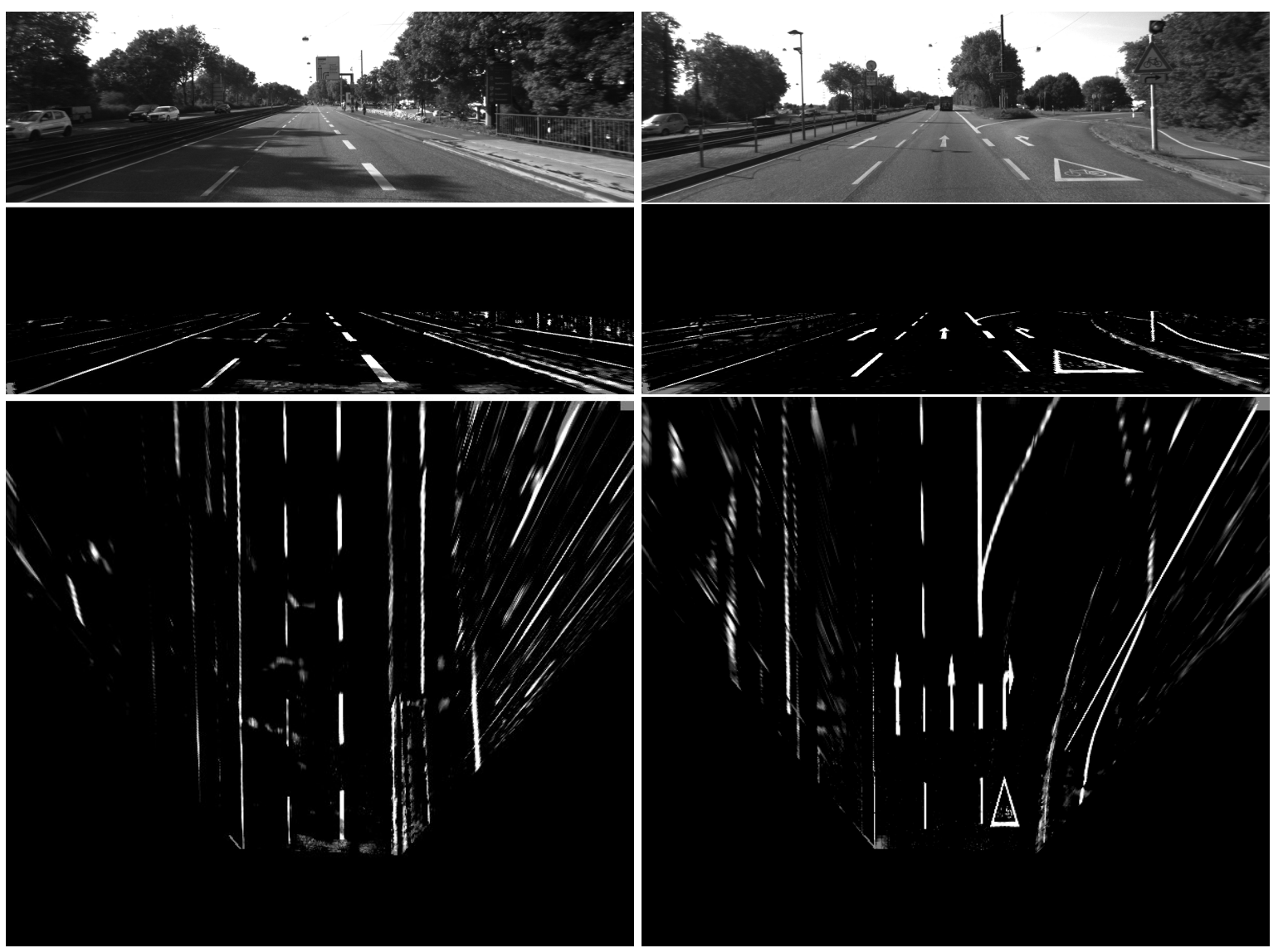

Figure 6.2: Upper image: shows the original image. Middle image: shows the same image after apply our method. Bottom image: shows the bird-eye-view image after apply the filter. Note that this filter is robust to shadows conditions.

steps, the first step performs the road marking enhancement described in section 6.1 followed by a Otsu's threshold method (Otsu, 1979) (Figure 6.3). The second step uses Probabilistic Hough Transform (Kiryati et al., 1991) in the binarized image to detect all possible lines that have at least a minimum number of votes and size. In this work, these lines are divided in two groups, left and right side, to compose the trapezoidal shape of ego-lane by an extrapolation process that create a full line segment of each side. The final part of this detection system estimates the ego-lane over the time in order to handle frames without road markings or with noise. More details about this method are available in (Batista et al., 2014), which presents an detailed evaluation for detection and estimation methods with datasets on several scenarios (Brazilian Scenarios).

Since the trapezoidal shape is estimated, it is possible to obtain the center of the lane. Since the camera is stuck in a fixed place on the middle of the vehicle, this system assumes that the center of image corresponds to exact half width of the front of vehicle. Thus, the difference in 

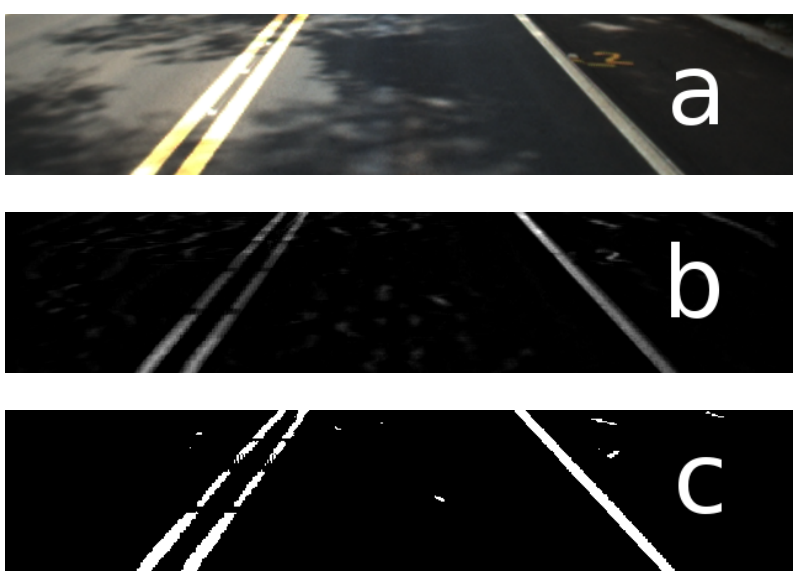

Figure 6.3: (a) ROI from the original image. (b) Image $M$ which has accumation from pixel's contrast. (c) Processed ROI image using a Otsu thresholding.

pixels between ideal trajectory and current car position can be computed and used by ADAS in order to alert the driver. The experiments were performed using $\operatorname{ROS}^{1}$ platform and OpenCV (Bradski e Kaehler, 2008) library. The visual results (detected ego-lane) are shown in Figure 6.4 and Figure 6.5 .
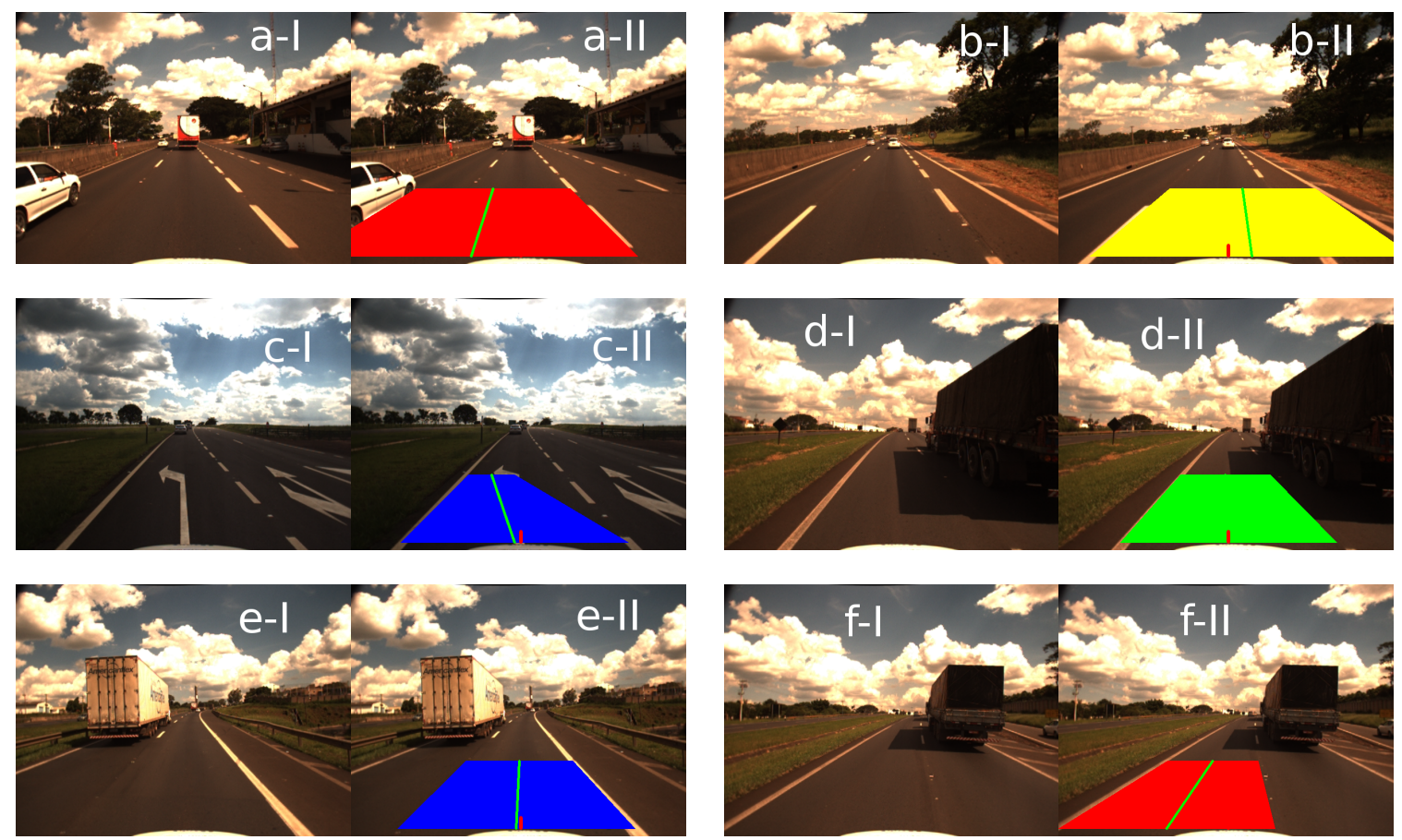

Figure 6.4: Images result from ego-lane detetion system in normal conditions

\footnotetext{
$1_{\text {WWW }}$ ros.org
} 

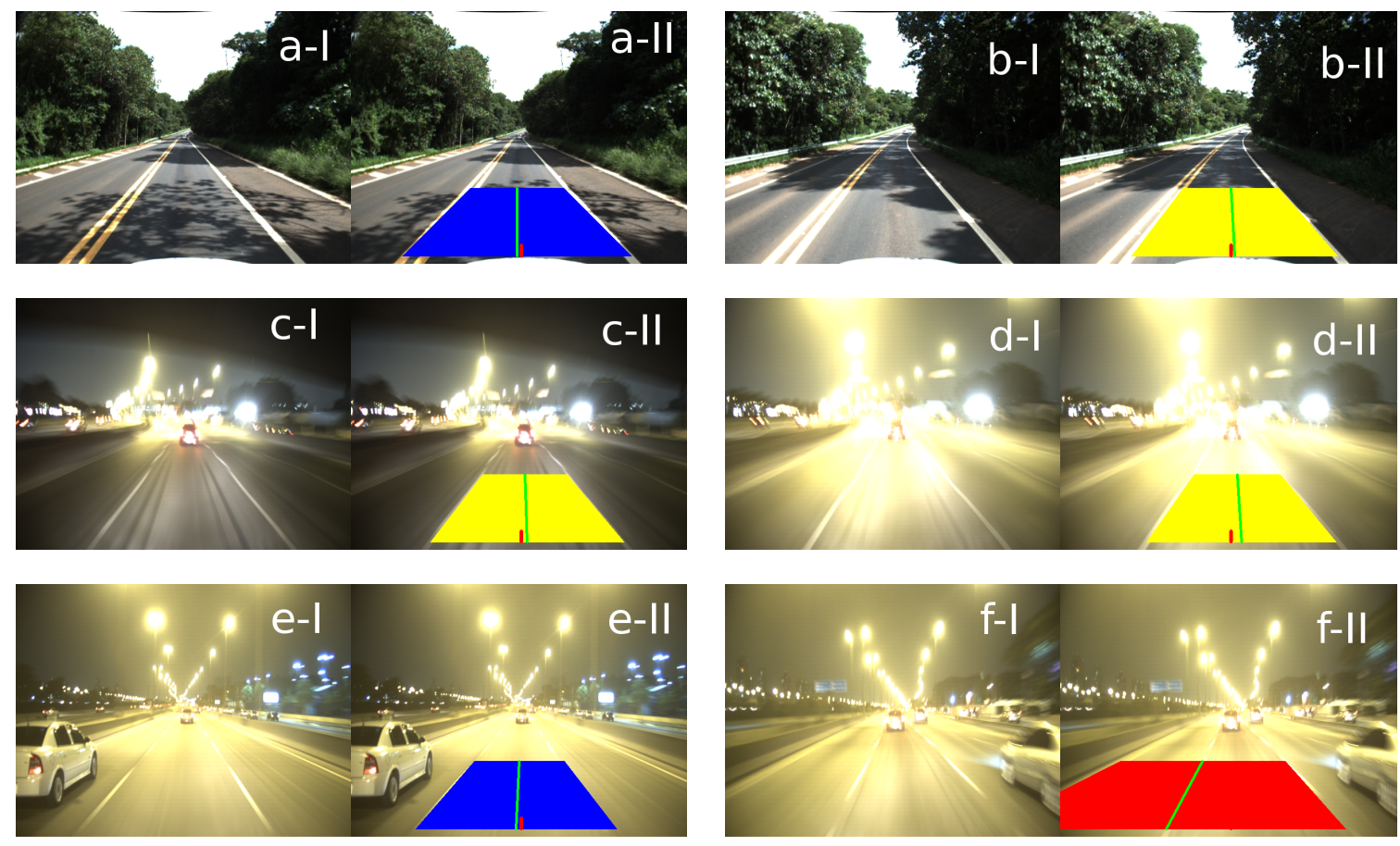

Figure 6.5: Images result from system in night situations and strong shadows occurences

In Figure 6.4 and Figure 6.5, images displayed with red lanes indicate that the center of the vehicle was 50 pixels or more distant from the center of a lane, indicating incorrect positioning of the vehicle. The yellow marked lanes indicate the vehicle is positioned slightly to the left and blue marked lanes similarly show a slightly right offset from the center of the lane. Some results shows failures where the system incorporates other elements in the lane shape (Figure 6.4(a) and (b), Figure 6.5(f)), which can be solved by incorporation of restrictions in width of lane and integrating of obstacle-detection system. Other images shows the robustness of the algorithm in different scenarios like traffic situations (Figure 6.4(d)), segmented road markers (Figure 6.4(e)) and night conditions (Figure 6.5) where the images shows situations with plenty of shade and brightness. The results indicates robustness to variation of illumination (including night images) and lane types. It was provided an alternate solution to Bird's Eye View approach allowing avoid any prior calibration dependency. This system needs just to set up the ROI(the position only, the size is constant) in front of the car, which is generally in the bottom part of the image. Therefore, the cameras can be quickly mounted on autonomous vehicles or assisted steering vehicles. 


\subsection{Automatic Semantic Waypoint Mapping}

In order to autonomous vehicles determine the optimal path to reach a destination, it is important to maintain the road network of the environment. Since a city is continuously expanding and changing, the road network also changes the number and position of traffic signs, lanes, and intersections. Therefore one of current issues for autonomous vehicles is the ability of a easy create and maintain precise and updated maps of urban environments. Automatic mapping system can help to solve this problem.

The road network is an environment representation that informs the traversable paths and may contain information about traffic signals and obstacles present in the streets. Basically, the road network map is formed by a set of waypoints that can be expanded to store semantic information as lanes, crosswalks and stop signs. This work proposed a road network map that automatically relates waypoints with any traffic information (Zoccoler et al., 2014). More specifically, we focused on the detection of roundabouts and lane structures in order to further combination with perception data (road estimation from previous chapters). We tested the proposed road network mapping using GPS data collected in real urban environments using the vehicle CaRINA-2 and the reconstruction of roundabouts and lanes were compared with publicly available satellite aerial maps.

Our road network mapping system converts the GPS points into more compact and suitable information for vehicle navigation. These structures allow us to classify each point with different semantic values, for instance, roundabouts points and lane points. In short, we eliminate points without losing world information by applying filters and detecting traffic structures. Also, specific tags can be appended to a waypoint to indicate location or traffic information.

Roundabout points can be detected by analyzing every waypoint and its successor in a list to identify closed-loops in the map. When the sum of all differences of orientation of a list reaches $2 \pi$, a roundabout candidate is created. After removal of redundant candidates, the optimal circle is computed by a two-dimensional circle fitting as described in (Shakarji, 1998). We define the cost function as the squared distance from each point to the calculated solution:

$$
J(x, y, r)=\sum_{i=W_{1}}^{W_{2}}\left(\sqrt{\left(x_{i}-C_{x}\right)^{2}+\left(y_{i}-C_{y}\right)^{2}}-r\right)^{2}
$$

where the pair $\left(C_{x}, C_{y}\right)$ represents the estimated circle center coordinates, $r$ is the estimated radius and $\left(x_{i}, y_{i}\right)$ are each sample point coordinates belonging to roundabout candidate. Since this is a non-linear problem for which no closed-form solutions can be derived, we choose the Gauss-Newton method with the Levenberg-Marquardt correction (Levenberg, 1944; Marquardt, 
1963). Also known as Levenberg-Marquardt method, this solution was adopted for its high rate of convergence and high probability of convergence given a random initial guess (Chernov e Lesort, 2005; Gander et al., 1994). The Levenberg-Marquardt minimization was performed through MINPACK library (Moré et al., 1980). A post-processing step detects and merge roundabout with same center point but different radius as shown in Figure. 6.6.
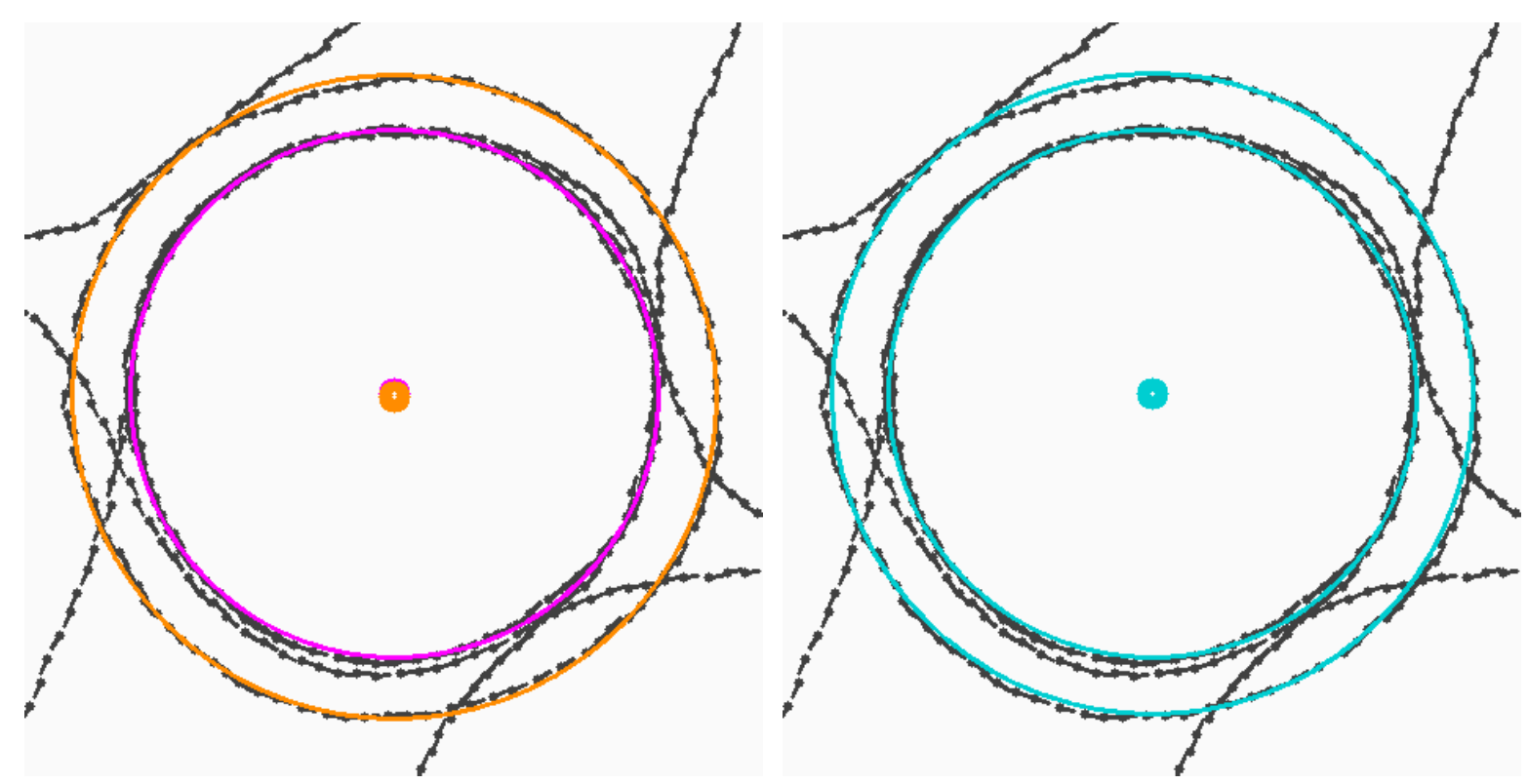

Figure 6.6: Levenberg-Marquardt fitting results. The orange and the magenta roundabout in 6.6a were collected at different times and have slightly different centers. In $6.6 \mathrm{~b}$ we perform the roundabout filtering to merge them into one Roundabout structure.

To detect lanes, the system split all points in several sub-lists using the detected roundabouts as divisor. If the remaining points does not belongs to roundabouts then it belongs to a sub-list that describes a single lane. For each lane, a filtering process is performed to remove redundant points in straight lines (see Figure 6.7), and to compute connections in intersection areas (between roundabouts and lanes).

The final result is a road network map (see Figure 6.8) in which can be used by several modules composing a complete and sophisticate system as a navigation system of an autonomous vehicle. Figure 6.8 firstly shows a 3D visualization of our robot over the road network map. Note that our map was previously created by our automatic system, i.e. no human intervention. The following images shows a visualization of our map with Velodyne points and a top-down view of part of our map. Figure 6.9 shows a visualization of a part of our map (around 5Km) in red at the corresponding satellite aerial map. 


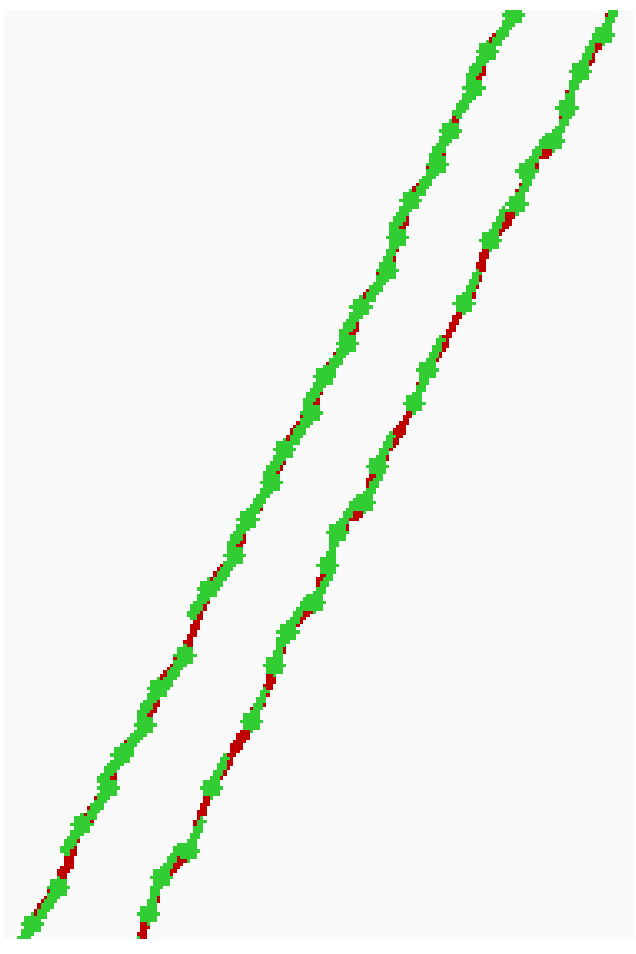

(a) Before lane filtering

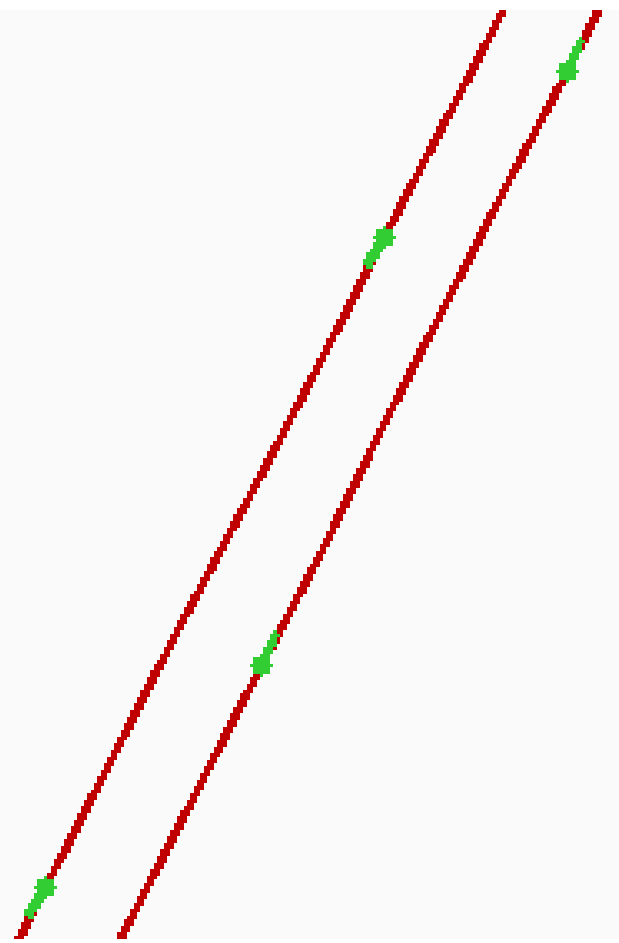

(b) After lane filtering

Figure 6.7: Lane noise reduction. The lane points are shown in green and the red lines represent the calculated trajectory.

This section presented a method for automatic construction of a waypoint map containing semantic information about roads. We developed the processing steps that implement flexible waypoint structures and help reducing the number of waypoints. More details about this project can be found in (Zoccoler et al., 2014). In the future, we plan to append more information to the semantic waypoint structures by integrating data collected from other sensors (road and lanes detected by our road estimation method). We will study detection solutions for cross intersections and cloverleaf interchanges. 


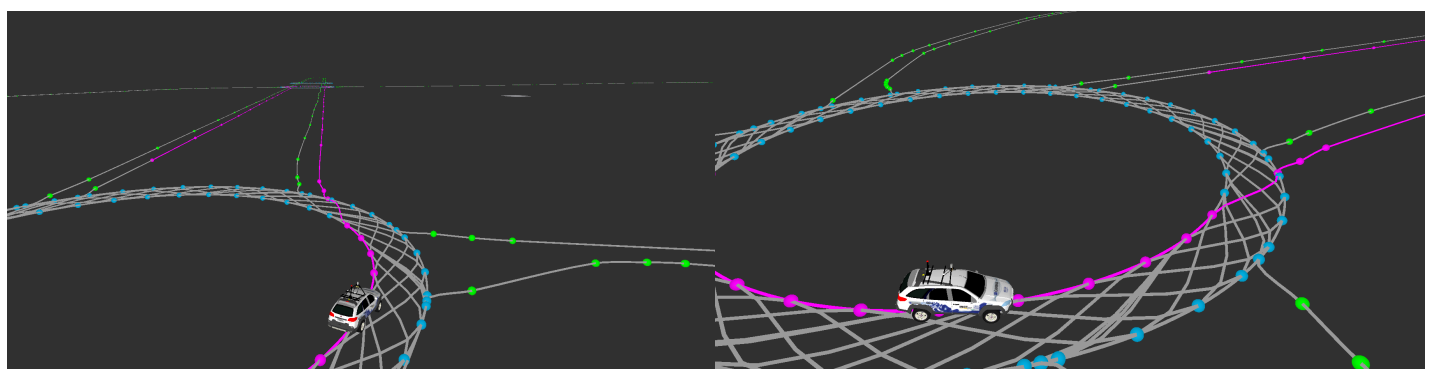

(a) 3D model in the mapped roundabout

(b) The purple line represents it current trajectory

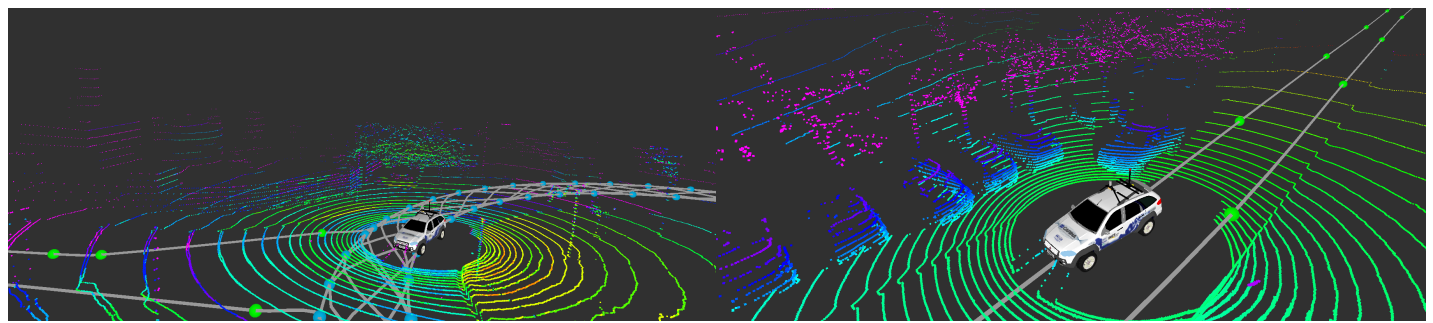

(c) Velodyne Points vied over the road map

(d) Parked Vehicles over road network map

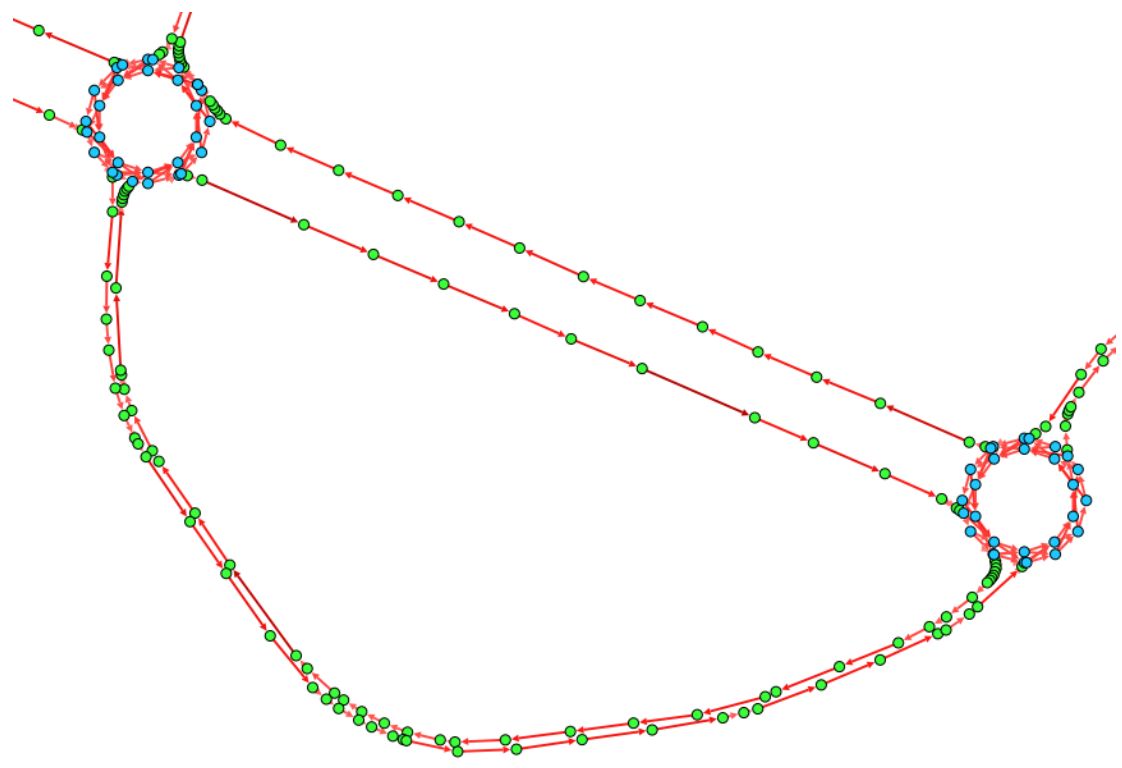

(e) A top down view of part of map

Figure 6.8: Campus II of USP Sao Carlos RVIZ view. The user can follow the 3D model of CaRINA II after selecting some point from our map. The current path is painted in purple.

\subsection{Navigation and Adaptive Cruise Control}

This work aimed to build a system to detect obstacles in front of the vehicle and depending on distance between them, our system must decrease the current velocity or even stop our 


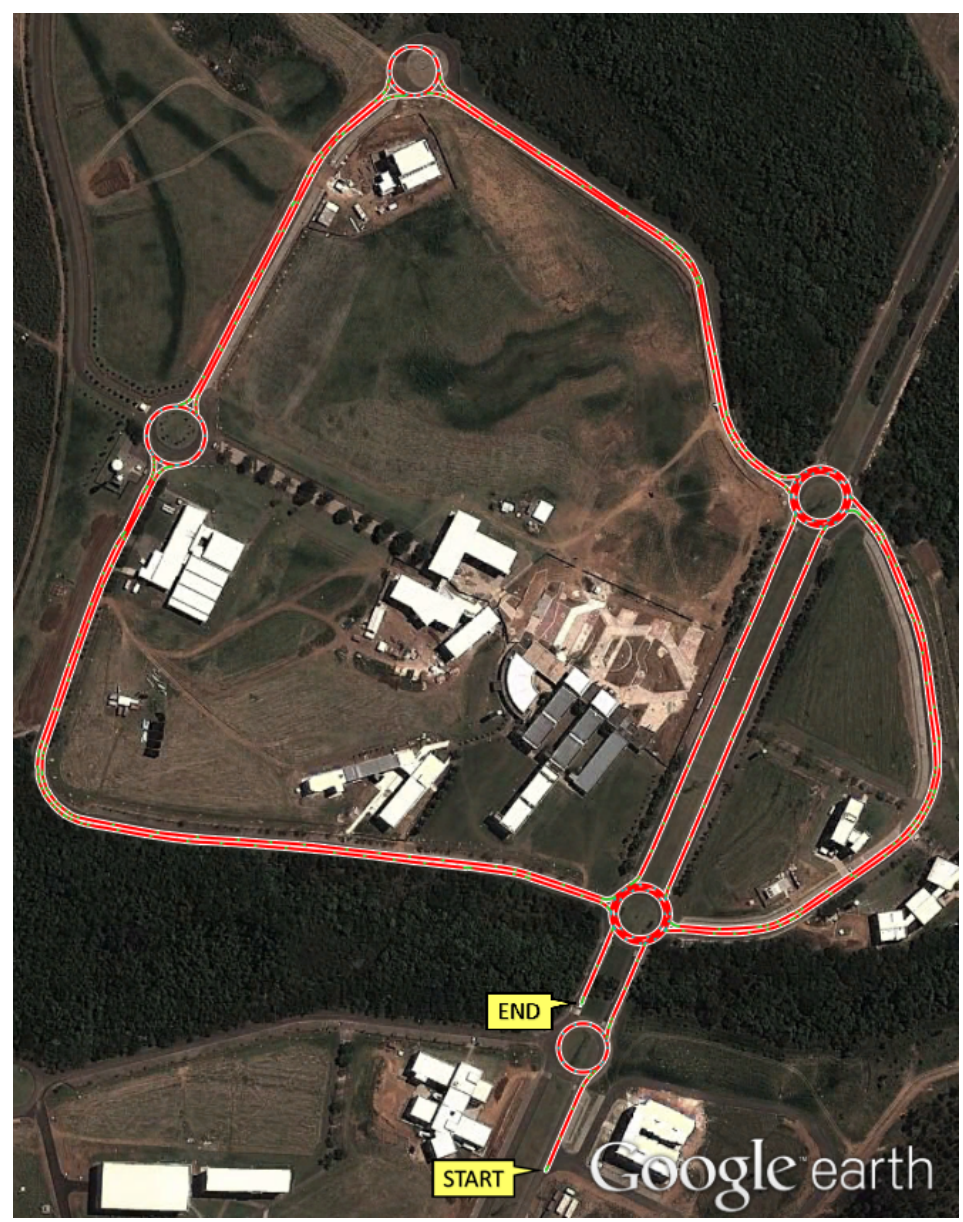

Figure 6.9: Campus II of USP Sao Carlos satellite view and resulting map shown in red.

vehicle if this distance is too small. This system is known in literature as adaptive cruise control (ACC), and our approach is to process information data from a 3D-LIDAR sensor, Velodyne HDL-32E, to create a data structure known as Range Image (RI). In this data structure, each pixel represents a $3 \mathrm{D}$ point projected in a cylindrical model where the main axis is the rotation axis of 3D-LIDAR. The value of each pixel is a distance value in the respective direction. Given a constant $\epsilon$, the number of columns ofthe RI is determined by $360 / \epsilon$, and all 3D information $(x, y, z)$ of a pixel $(u, v)$ can be recovered by Equation 6.3:

$$
\left(\begin{array}{l}
x \\
y \\
z
\end{array}\right)=\left(\begin{array}{c}
-d * \cos (\text { angle }[u]) * \sin (v * \epsilon) \\
-d * \sin (\text { angle }[u]) * \cos (v * \epsilon) \\
d * \sin (\text { angle }[u])
\end{array}\right)
$$


where $d$ is pixel value in distance, angle[] is a vector that store an angle for each emitter, angle $[u]$ is the angle of $u$-th laser emitter, $v$ is the column index, and $\epsilon$ is the chosen constant that defines the horizontal precision.

This RI is used as input by obstacle detection method presented in this thesis that uses only local information to classify each pixel in obstacle or non-obstacle (see Figure 6.10). The neighbourhood chosen is image processing like 8-connected, therefore a pixel $(u, v)$ has a set of neighbours described in Eq. 6.4. The result of obstacle detection is showed in Fig. 6.10 where white pixels are obstacles while black pixels are non-obstacles.

$$
\begin{array}{ccc}
n_{1}=(u-1, v-1) & n_{2}=(u-1, v) & n_{3}=(u-1, v+1) \\
n_{4}=(u, v-1) & \mathbf{p}=(\mathbf{u}, \mathbf{v}) & n_{5}=(u, v+1) \\
n_{6}=(u+1, v-1) & n_{7}=(u+1, v) & n_{8}=(u+1, v+1)
\end{array}
$$

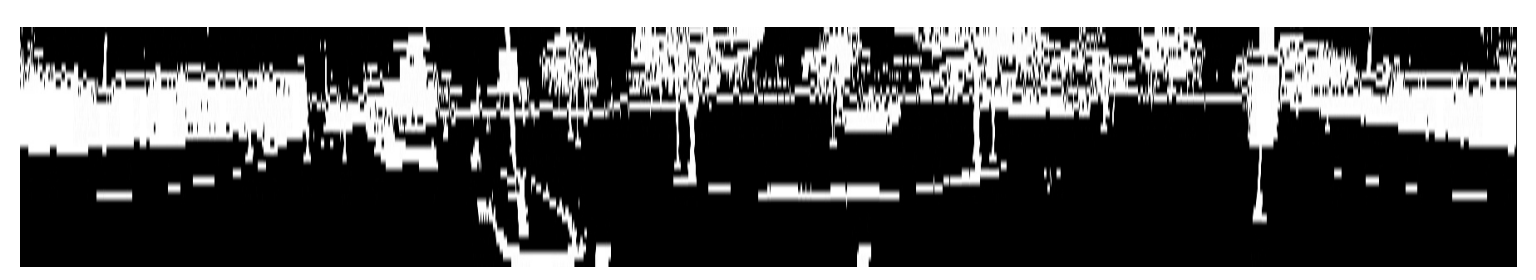

Figure 6.10: Results of obstacle detection method in a range image. White pixels are obstacles and black pixels otherwise.

The next step is to build a virtual 2D-LIDAR sensor with $360^{\circ}$ field of view (VirtualScan) that projects all closest obstacles around the vehicle (see Figure 6.11, VirtualScan in red points). The array of distances of this VirtualScan is used to verify if there is an obstacle in front the vehicle and to estimate the distance between them, after compute this information (perception status), an action is chosen to be executed.

Inside Carina-2, all the processes are executed in parallel as different modules, i.e. while one module is responsible by detection of obstacles, another module computes the required actions (steering, throttle and brake) to drive our platform to the goal. Therefore, after classifying the range image and verify if exists an obstacle in front of the vehicle, a supervisor module receives, at same time, an "expected action" and a "perception status". If an obstacle exists in front of our vehicle, the maximum velocity in meters by second is computed by our ADAS using Equation 6.5:

$$
\text { vel }=\left\{\begin{array}{cc}
\frac{d-5.0}{2.0} & \text { if } d>\kappa \\
0 & \text { otherwise }
\end{array}\right.
$$




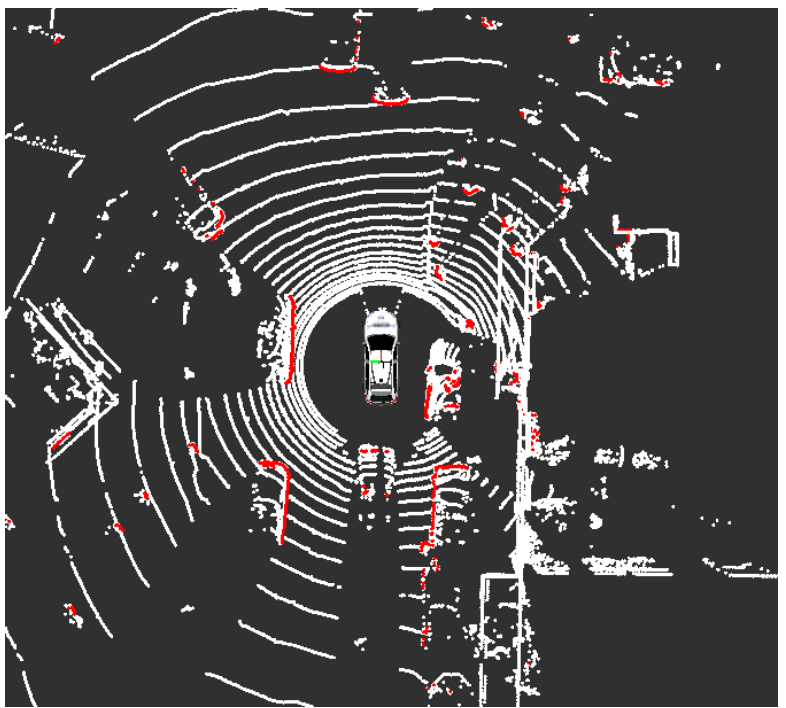

(a) UpDown View

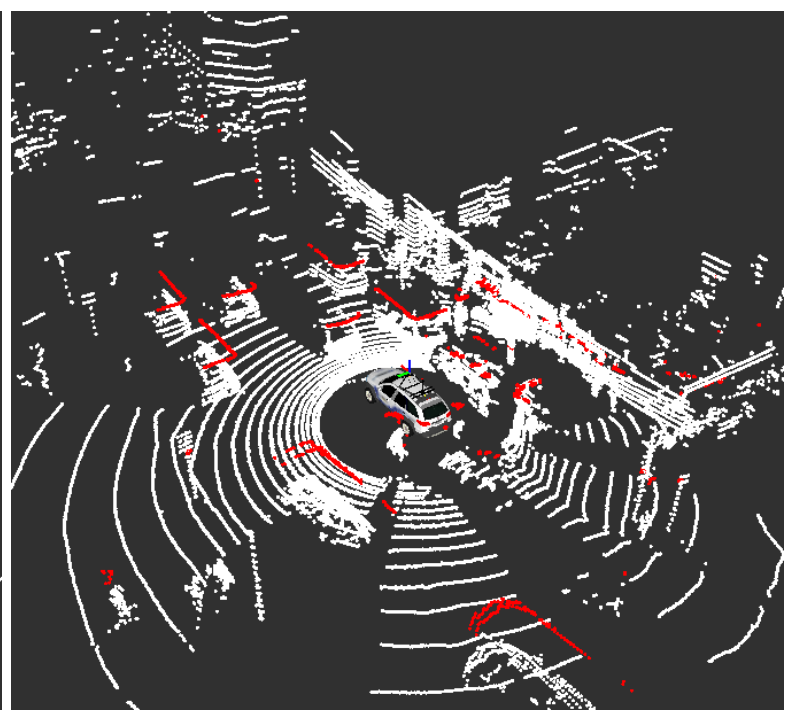

(b) Perspective view

Figure 6.11: Vizualization of VirtualScan in red over all white points from 3D-LIDAR Velodyne HDL-32E. Note that white points are in 3D coordinates while red points are in $2 \mathrm{D}$ coordinates.

where $d$ is the distance of the closest obstacle in front of our vehicle, $\kappa$ is the minimum distance allowed between our vehicle and one obstacle. If the current velocity is lower than vel, the supervisor module send the current action to CaRINA-2 execute. If not but vel $>0$ then the supervisor module starts to reduce the current velocity activating the brake pedal. If vel $=0$ then the brake pedal is fully activated.

This section presented an ACC based on the use of 3D-LIDAR sensor to detect a obstacle in front of our vehicle. Due to the high cost of this kind of sensor, this sensor is not considered viable for the industry. As the algorithm is based in a data structure that can be used in other kind of sensors, a stereo image sensor approach can be used in order to reduce a big part of the cost of this system. The method used in this ACC showed high robustness since it was used in the experimental demonstration performed in October of 2013 (Fig. 6.12). This experimental demonstration, to the best of our knowledge was the first commercial vehicle from Latin America capable of performing autonomous navigation in real streets and it was showed in media as Globo ${ }^{2}, \mathrm{SBT}^{3}$, WebMotors ${ }^{4}$ and FAPESP's Magazine ${ }^{5}$. The video of FAPESP's Magazine

\footnotetext{
${ }^{2}$ http: / / www youtube. com/watch?v=v7sQYNWLbLg

${ }^{3}$ http: / / www youtube. com/watch? v=NND 7x5EDp28

${ }^{4}$ http: / / www youtube. com/wat ch? v=LnzyaqmenPo

${ }^{5}$ http: // www youtube. com/watch? $\mathrm{v}=3 \mathrm{chjXuVx} 8 \mathrm{Fs}$
} 

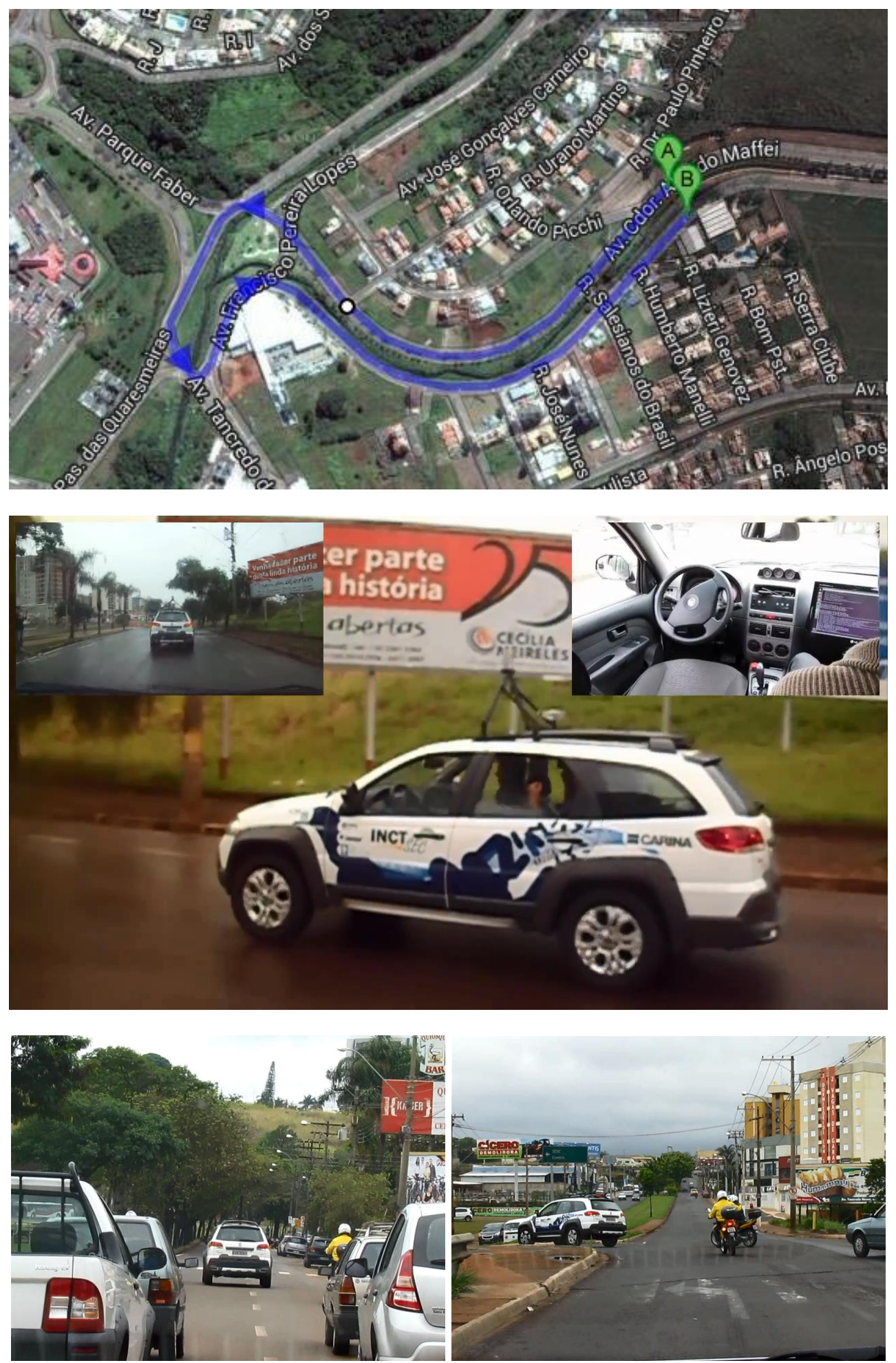

Figure 6.12: Pictures of public demonstration of CaRINA-2. 
shows with more details the execution of our ACC, where the vehicle stopped to give preference to pedestrians.

\subsection{Considerations}

This chapter presented several contributions as road marking enhancement, a lane departure warning, adaptive cruise control and an automatic mapping system that are and will be part of project CaRINA-2. All these works are a directly ramification of the work presented in this thesis and they were developed in cooperation with other members from LRM. 


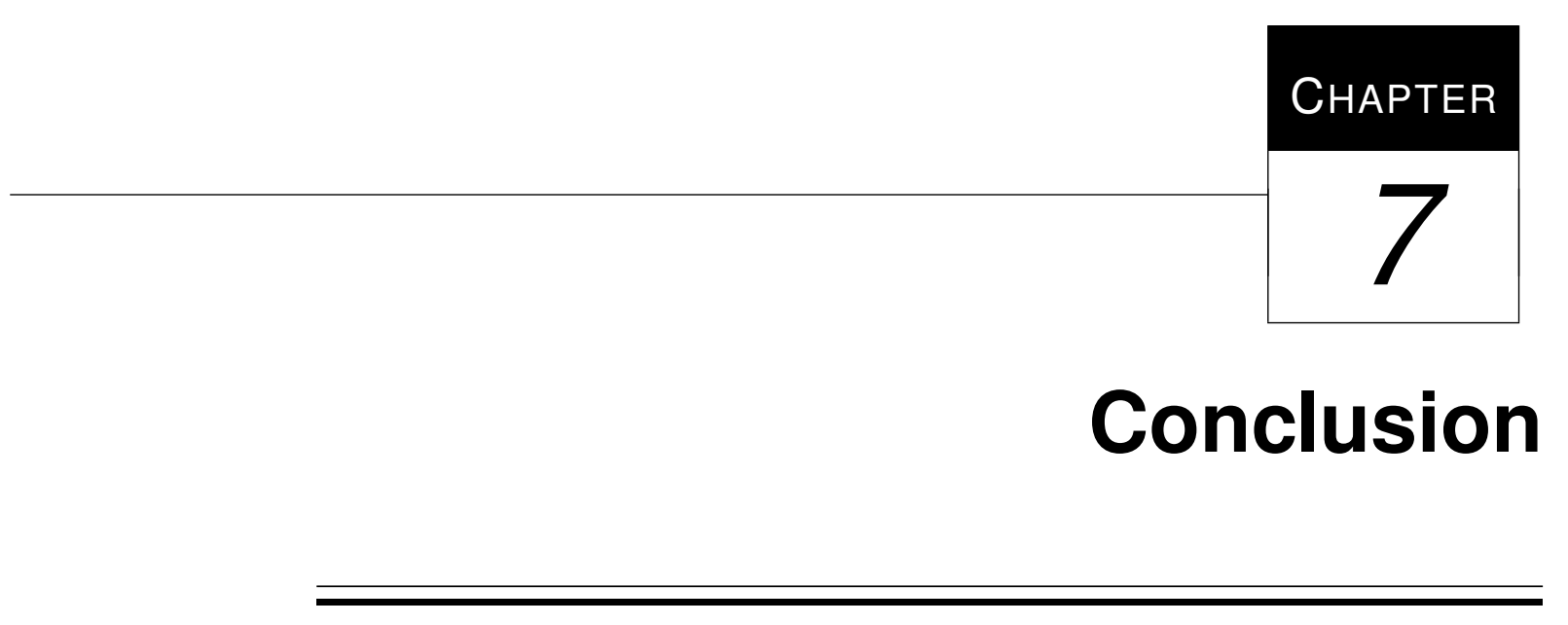

Advanced Driver Assistance Systems (ADAS) and Self-driving cars aim at reducing the large number of deaths and injuries caused by traffic accidents. Therefore, several universities and companies have been researching into these topics and they achieved impressive results that show the viability of this technology for the next years. Among the various issues related to this technology, perception systems as obstacle and road detection are still unsolved problems. To date, sophisticated systems use high-cost sensors like 3D-LIDAR and/or adopt, in majority, machine learning methods which have impressive results if they were trained with good training-datasets. Since it is a complex and expensive job to create and maintain databases of road scenarios, adaptive and/or self-supervised methods are good candidates for robust detection systems for the future. Due to that, this thesis presented a method to estimate obstacles and roads using low cost sensors. It avoids machine learning techniques and the most common assumptions presented in literature. These methods can be used as a pre-processing step to improve and allow adaptive methods of learning.

The main contribution presented in this work is a road estimation using stereo camera systems. To create a general approach, a fast obstacle estimation method was developed with capability to detect several type of obstacles avoiding several assumptions like structured obstacles, minimum height and other. This obstacle estimation has as output an obstacle-confidence value for each pixel and can be used by other systems such as detection and tracking of obstacles as shown in Figure 7.1. Furthermore, the development of these methods also generates several 
other single approach contributions such as the use of the projection of point clouds in screen planes to create a local-relationship allowing detection of road area and obstacles without several common assumptions. The proposed system was evaluated using different combinations of sensors by a standard benchmark. It successfully detected the road (paved free space) in multiple scenarios without previous training. The proposed system requires only one major assumption which is the road area it is delimited by obstacles (curbs included) instead of assumptions about road shape. Furthermore it does not require previous selection of threshold values for the height and size of obstacles, and no other parameters, such as cell size value, differently from grid and voxel-based approaches.

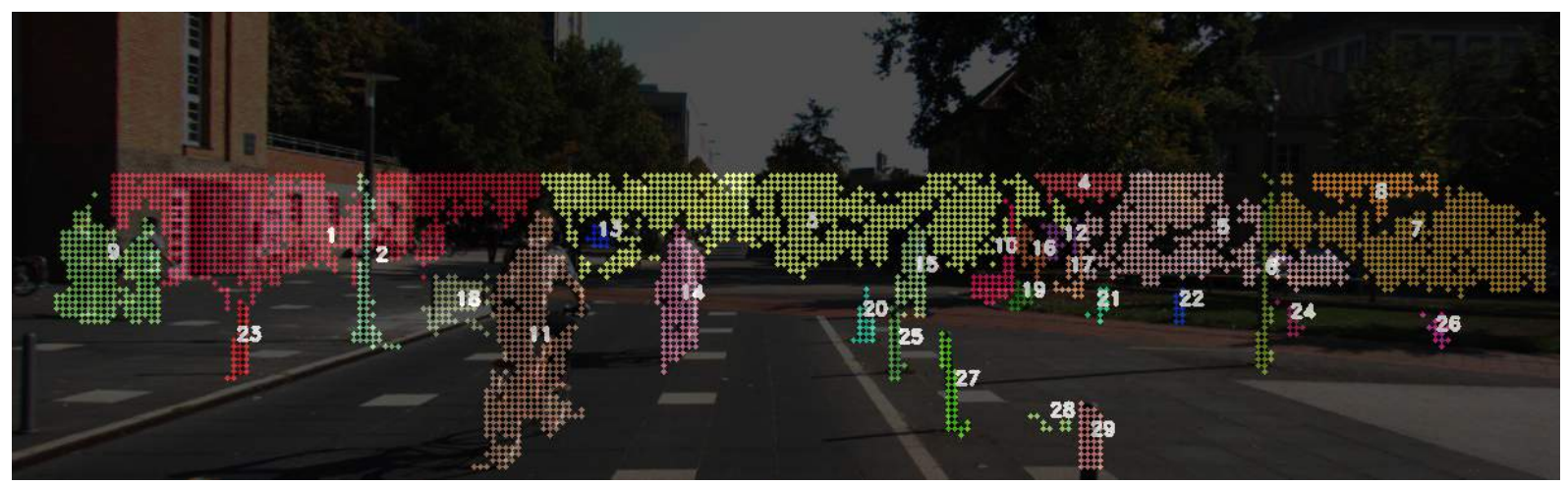

Figure 7.1: Example of future work: Detection and tracking of moving obstacles.

Taking advantage of the "sparsity approach", this thesis also shows the possibility of replacing a high cost sensor as 3D LIDAR by a low cost stereo image sensor. The main feature is the low runtime because our modified version of correspondence algorithm does not compute and not depends on dense disparity map as the most common methods in literature.

\subsection{Future Directions}

As future work to our road estimation, we aim at integrating visual information, like edges or texture. The replacement of our constants $\theta$ and $\lambda$ by functions to handle perspective effect will improve the range of view of our method (a pre-calibration process can provide these functions). The integration with uncertainties of disparity values available in some disparity algorithms also can improve the obstacle and road detection methods.

Since one of the current issues for autonomous vehicles is the ability of a easily creation and maintenance of precise and updated maps of urban environments, the proposed road estimation method can be used to compose an automatic mapping system of road network, since it does 
not need previous training. However, before combining and storing a huge amount of data as a grid map, we can take advantage of the knowledge of the known path to integrate semantic information like lanes into the perception system. This integration allows generation of accurate maps in a data-structure that combine topological information with metric information.

Given the road estimation output and previous calibration matrix it is possible to create a 2D map of road pixels only. Since all obstacles points already are removed, we can interpolate points along all edges that belongs to the graph that connects just road points. The result is a dense $3 \mathrm{D}$ point cloud from the road or a $2 \mathrm{D}$ map very similar to bird eye view images. If it is combined with a good localization system, visual odometry and/or high precision GPS, it is possible to accumulate processed frames over time and generate a grid map of road. Also, it is possible to highlight, for example, all road markings as results that can be viewed on Figs.7.2.

Using this map with detected obstacles, we are able to combine different informations like curbs and difference of color between road and side-walks to get an estimation of the border of the road. Note, that this system will not need any previous information about geometry, colors or texture from road (everything can be adaptive). This work also can be combined with road network structure presented in (Zoccoler et al., 2014) in order to initialize all possible lanes and confirm its existence by the road markings as well as detect other urban elements like pedestrian crosswalk and driveway. 

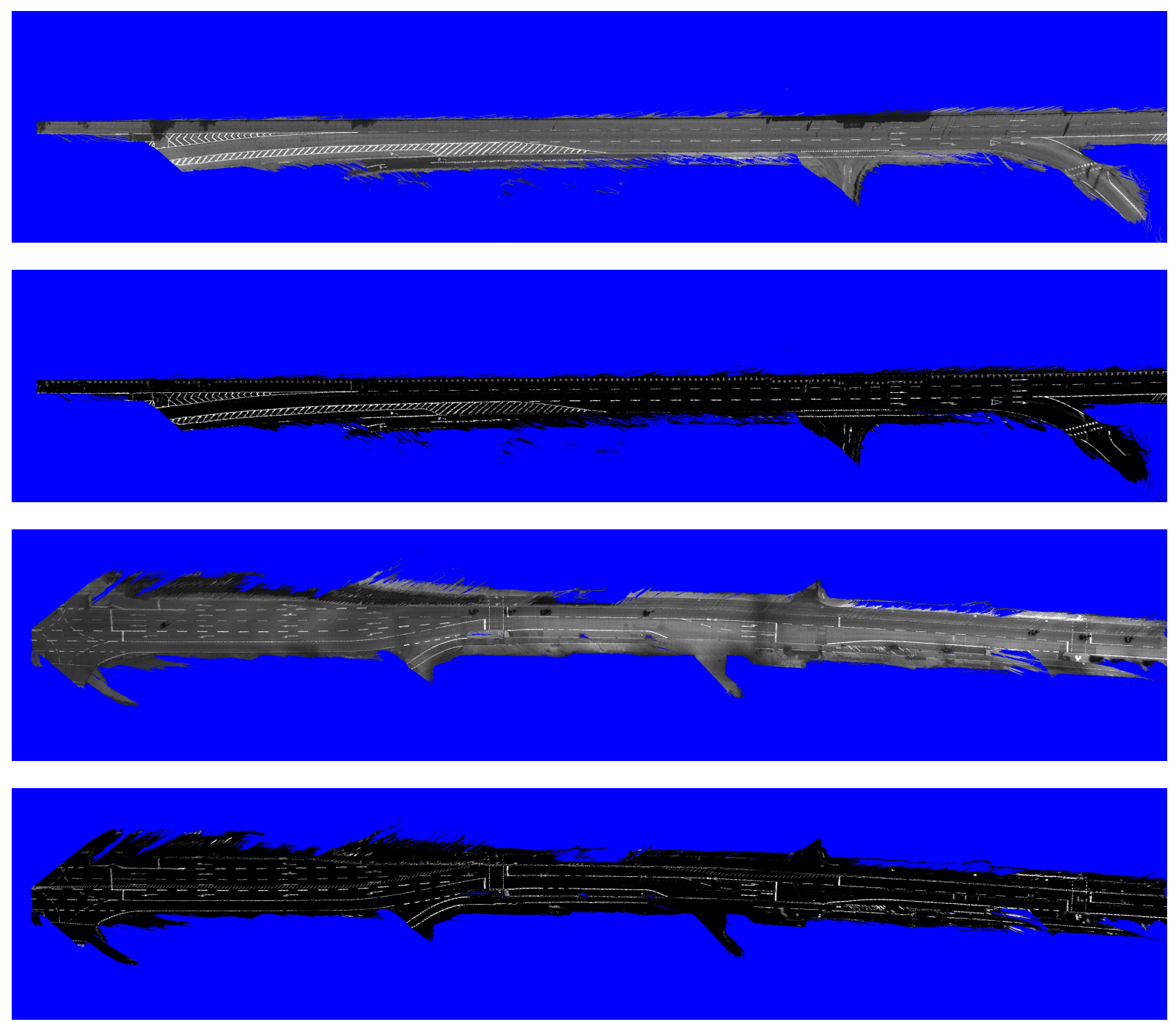

Figure 7.2: $2 \mathrm{D}$ map created with just a single frame of gray-scale image and 3D points generated by a 3D-LIDAR. 


\section{Bibliography}

A. Miranda Neto, A.C. Victorino, I. F. J. F. Real-time collision risk estimation based on pearson's correlation coefficient. In: IEEE Workshop on Robot Vision, 2013, p. 1 - 6.

Aufrere, R.; Aufrère, R.; Mertz, C.; Thorpe, C. Multiple sensor fusion for detecting location of curbs, walls, and barriers. In: Proceedings of the IEEE Intelligent Vehicles Symposium (IV2003, 2003.

AUTONOMOSLABS Artificial vision and intelligent systems laboratory. http:// autonomos.inf.fu-berlin.de/, visitado em Abril, 2012.

Bar Hillel, A.; Lerner, R.; LeVi, D.; RAZ, G. Recent progress in road and lane detection: a survey. Machine Vision and Applications, p. 1-19, 2012.

Disponível em http://dx.doi.org/10.1007/s00138-011-0404-2

Batista, M. P.; Y., S. P.; Wolf, D. F.; Gomes, D. Lane detection and estiamtion using perspective image. In: Robotic Symposium, 2014. LARS 14. IEE Latin American, 2014.

Bertozzi, M.; Broggi, A. Gold: a parallel real-time stereo vision system for generic obstacle and lane detection. Image Processing, IEEE Transactions on, v. 7, n. 1, p. 62 -81, 1998.

Bertozzi, M.; Broggi, A.; Fascioli, A. Vision-based intelligent vehicles: State of the art and perspectives. Robotics and Autonomous Systems, v. 32, n. 1, p. 1-16, 2000. 
Bonin-Font, F.; Ortiz, A.; Oliver, G. Visual navigation for mobile robots: A survey. Journal of Intelligent \& Robotic Systems, v. 53, p. 263-296, 10.1007/s10846-008-9235-4, 2008.

Borenstein, J.; Koren, Y. The vector field histogram-fast obstacle avoidance for mobile robots. Robotics and Automation, IEEE Transactions on, v. 7, n. 3, p. 278-288, 1991.

Bosch Bosch. http://www.bosch-mobility-solutions.com/en/de/ _technik/component/CO_PC_DA_Road-Sign-Recognition_CO_PC_ Driver-Assistance_2563.html?compId=5442, 2014.

BRADSKI, G.; KAEHLER, A. Learning opencv: Computer vision with the opencv library. O’Reilly, 2008.

Broggi, A.; Bertozzi, M.; FAscioli, A. Argo and the millemiglia in automatico tour. Intelligent Systems and their Applications, IEEE, v. 14, n. 1, p. 55 -64, 1999.

Buehler, M.; Iagnemma, K.; Singh, S. The 2005 darpa grand challenge: The great robot race. 1st ed. Springer Publishing Company, Incorporated, 2007.

Buehler, M.; IAgnemma, K.; Singh, S. The darpa urban challenge: Autonomous vehicles in city traffic. 1st ed. Springer Publishing Company, Incorporated, 2009.

CAPES Coordination for the improvement of higher level -or education- personnel. http: //www.capes.gov.br, 2014.

Chernov, N.; Lesort, C. Least squares fitting of circles. J. Math. Imaging Vis., v. 23, n. 3, p. 239-252, 2005.

CIE International commission on illumination. http://www.cie.co.at/, 2014.

CNPQ National council for scientific and technological development. http://www . cnpq. br, 2014.

Crisman, J.; Thorpe, C. Unscarf, a color vision system for the detection of unstructured roads. In: Proceedings of IEEE International Conference on Robotics and Automation, 1991, p. $2496-2501$.

D.A. LiMA, G. P. Stereo vision system for autonomous vehicle navigation into environments with obstacles (original text in portuguese). In: Congresso Brasileiro de Automatica, 2010, p. $224-231$. 
Dagum, L.; Menon, R. Openmp: an industry standard api for shared-memory programming. Computational Science \& Engineering, IEEE, v. 5, n. 1, p. 46-55, 1998.

DAIMLER Daimler. http://www.daimler.com/dccom/ 0-5-658451-1-1550372-1-0-0-0-0-0-13-7145-0-0-0-0-0-0-0.html, 2014.

DesouZA, G.; KAK, A. Vision for mobile robot navigation: a survey. Pattern Analysis and Machine Intelligence, IEEE Transactions on, v. 24, n. 2, p. 237 -267, 2002.

DickMANns, E. D. Vehicles capable of dynamic vision: a new breed of technical beings? Artif. Intell., v. 103, p. 49-76, 1998.

ELROB Eureopean land-robot trial. http: / / www.elrob.org, visitado em Abril, 2012.

Enzweiler, M.; Greiner, P.; KnOpPel, C.; Franke, U. Towards multi-cue urban curb recognition. In: Intelligent Vehicles Symposium (IV), 2013 IEEE, 2013, p. 902-907.

EUROFOT European field operational test. http://www.eurofot-ip.eu, visitado em Abril, 2012.

FAPESP São paulo research fundation. http://www. fapesp.br, 2014.

Forsyth, D. A.; Ponce, J. Computer vision: A modern approach. Prentice Hall, 2002.

Fritsch, J.; Kuehnl, T.; Geiger, A. A new performance measure and evaluation benchmark for road detection algorithms. In: International Conference on Intelligent Transportation Systems (ITSC), 2013.

Gander, W.; Golub, G.; Strebel, R. Least-squares fitting of circles and ellipses. BIT Num. Math., v. 34, n. 4, p. 558-578, 1994.

GCDC Grand cooperative driving challenge. http: //www. gcdc . net, visitado em Abril, 2012.

Geiger, A.; Lenz, P.; Stiller, C.; Urtasun, R. Vision meets robotics: The KITTI dataset. International Journal of Robotics Research (IJRR), v. 32, p. 1229-1235, 2013.

Geiger, A.; LenZ, P.; URTASUn, R. Are we ready for autonomous driving? the kitti vision benchmark suite. In: Conference on Computer Vision and Pattern Recognition (CVPR), 2012a. 
Geiger, A.; Moosmann, F.; Car, O.; Schuster, B. Automatic calibration of range and camera sensors using a single shot. In: International Conference on Robotics and Automation (ICRA), 2012b.

Geiger, A.; Roser, M.; Urtasun, R. Efficient large-scale stereo matching. In: ACCV, 2010.

Google The official google blog, 2010. http://googleblog.blogspot.com/ 2010/10/what-were-driving-at.html by Sebastian Thrun, visitado em Abril, 2012.

Google Googleselfdrivingcar. https://plus.google.com/ +GoogleSelfDrivingCars/videos, 2014.

Gregor, R.; Lutzeler, M.; Pellkofer, M.; Siedersberger, K.; Dickmanns, E. Ems-vision: a perceptual system for autonomous vehicles. In: Intelligent Vehicles Symposium, 2000. IV 2000. Proceedings of the IEEE, 2000, p. 52 -57.

H. Roncancio, A.C. Hernandes, M. B. Vision-based system for pedestrian recognition using a tuned svm classifier. In: IEEE Workshop on Engineering Applications, 2012, p. 1 6.

HAVEIT Highly automated vehicles for intelligent transport. http://www . haveit-eu . org, visitado em Abril, 2012.

He, Y.; WAnG, H.; Zhang, B. Color-based road detection in urban traffic scenes. Intelligent Transportation Systems, IEEE Transactions on, v. 5, n. 4, p. 309-318, 2004.

Hirschmueller, H. Stereo processing by semiglobal matching and mutual information. PAMI, v. 30, p. 328-41, 2008.

HÄSElich, M.; Arends, M.; LAng, D.; PAulus, D. Terrain classification with markov random fields on fused camera and 3d laser range data. In: 5th European Conference on Mobile Robotics (ECMR), Proceedings of the, Örebro University, 2011, p. 153-158.

JUNG, C. R.; KELBER, C. R. Lane following and lane departure using a linear-parabolic model. Image Vision Comput., v. 23, n. 13, p. 1192-1202, 2005.

KAMMEL, S.; PITZER, B. Lidar-based lane marker detection and mapping. In: Intelligent Vehicles Symposium, 2008 IEEE, 2008, p. 1137-1142. 
Kiryati, N.; Eldar, Y.; Bruckstein, A. A probabilistic hough transform. Pattern Recognition, v. 24, n. 4, p. 303-316, 1991.

Kong, H.; Audibert, J.-Y.; Ponce, J. Vanishing point detection for road detection. In: Computer Vision and Pattern Recognition, 2009. CVPR 2009. IEEE Conference on, 2009, p. 96-103.

Kong, H.; Audibert, J.-Y.; Ponce, J. General road detection from a single image. Image Processing, IEEE Transactions on, v. 19, n. 8, p. 2211-2220, 2010.

Kuehnl, T.; Kummert, F.; Fritsch, J. Spatial ray features for real-time ego-lane extraction. In: Proc. IEEE Intelligent Transportation Systems, 2012.

L. MG, L.M. HonORIO, M. R. Modeling path searching for local navigation of an intelligent autonomous vehicle (original text in portuguese). In: Congresso Brasileiro de Automatica, 2010.

L. Veronese, L.J. Lyno Jr., J. O. N. A. F. C. B. A. D. S. Parallel implementations of the csbp stereo vision algorithm. In: Simposio em Sistemas Computacionais, 2011, p. 12 - 21.

Lee, D. T.; Schachter, B. J. Two algorithms for constructing a Delaunay triangulation. International Journal of Parallel Programming, v. 9, n. 3, p. 219-242, 1980.

Levenberg, K. A Method for the Solution of Certain Non-linear Problems in Least Squares. Quart. Appl. Math., v. 2, p. 164-168, 1944.

De Lima, D.; Pereira, G. Navigation of an autonomous car using vector fields and the dynamic window approach. Journal of Control, Automation and Electrical Systems, v. 24, n. 1-2, p. 106-116, 2013.

Disponível em http://dx.doi.org/10.1007/s40313-013-0006-5

L.L.G. Veermas, L.M. Honorio, M. V. L. M. A methodology for supervised learning applied to intelligent vehicles (original text in portuguese). In: Congresso Brasileiro de Automatica, 2010.

LRM Mobile robotics laboratory. http://www. lrm.icmc.usp.br, 2014.

M. Heinen, F. Osorio, F. H. C. K. Seva3d: using artificial neural networks to autonomous vehicle parking control. In: IEEE International Joint Conference on Neural Networks IJCNN'06, 2006, p. $4704-4711$. 
Mallot, H.; BÜlthoff, H.; Little, J.; Bohrer, S. Inverse perspective mapping simplifies optical flow computation and obstacle detection. Biological Cybernetics, v. 64, n. 3, p. 177-185, 1991.

Disponível em http://dx.doi.org/10.1007/BF00201978

MARquardT, D. W. An Algorithm for Least-Squares Estimation of Nonlinear Parameters. SIAM Journal on Applied Mathematics, v. 11, n. 2, p. 431-441, 1963.

Matthies, L.; Kelly, A.; Litwin, T.; Tharp, G. Obstacle detection for unmanned ground vehicles: a progress report. In: Intelligent Vehicles '95 Symposium., Proceedings of the, 1995, p. 66-71.

Matthies, L.; Litwin, T.; Owens, K.; Rankin, A.; Murphy, K.; Coombs, D.; Gilsinn, J.; Hong, T.; Legowik, S.; NAshman, M.; Billibon Performance evaluation of ugv obstacle detection with ccd/flir stereo vision and ladar. In: IEEE ISIC/CIRA/ISAS Joint Conference, 1998.

MERCEDES Mercedes. http://www.mercedes-benz.com/en/mercedes-benz/ innovation/ces-2015/, 2015.

Mohan, R. Deep deconvolutional networks for scene parsing. 2014.

Moosmann, F.; Pink, O.; Stiller, C. Segmentation of 3d lidar data in non-flat urban environments using a local convexity criterion. In: Proceedings of the IEEE Intelligent Vehicles Symposium, Xi'an, China, 2009, p. 215-220.

Moosmann, F.; Stiller, C. Joint self-localization and tracking of generic objects in 3d range data. In: Proceedings of the IEEE International Conference on Robotics and Automation, Karlsruhe, Germany, 2013, p. 1138-1144.

Moré, J. J.; Garbow, B. S.; Hillstrom, K. E. User Guide for MiNPACK-1. ANL-80-74, Argonne National Laboratory, 1980.

Oniga, F.; Nedevschi, S.; Meinecke, M. M. Curb detection based on a multi-frame persistence map for urban driving scenarios. In: Intelligent Transportation Systems, 2008. ITSC 2008. 11th International IEEE Conference on, 2008, p. 67-72.

Otsu, N. A Threshold Selection Method from Gray-level Histograms. IEEE Transactions on Systems, Man and Cybernetics, v. 9, n. 1, p. 62-66, 1979.

Disponível em http://dx.doi.org/10.1109/tsmc.1979.4310076 
SChARSTEIN, D.; SzEliski, R. A taxonomy and evaluation of dense two-frame stereo correspondence algorithms. Int. J. Comput. Vision, v. 47, n. 1-3, p. 7-42, 2002.

ShAKARJI, C. M. Least-Squares Fitting Algorithms of the NIST Algorithm Testing System. Journal of Research of the National Institute of Standards and Technology, v. 103, n. 6, p. 633-641, 1998.

ShIn, Y.; JUnG, C.; ChUnG, W. Drivable road region detection using a single laser range finder for outdoor patrol robots. In: Intelligent Vehicles Symposium (IV), 2010 IEEE, 2010, p. $877-882$.

Shinzato, P.; Wolf, D.; Stiller, C. Road terrain detection: Avoiding common obstacle detection assumptions using sensor fusion. In: Intelligent Vehicles Symposium Proceedings, 2014 IEEE, 2014a, p. 687-692.

ShinZato, P. Y.; Gomes, D.; Wolf, D. F. Road estimation with sparse 3d points from stereo data. In: Intelligent Transportation Systems (ITSC), 2014 IEEE 17th International Conference on, 2014b, p. 1688-1693.

Shinzato, P. Y.; Grassi JR., V.; Osório, F. S.; Wolf, D. F. Fast visual road recognition and horizon detection using multiple artificial neural networks. In: Intelligent Vehicles Symposium, 2012, p. 1090-1095.

Siegemund, J.; Pfeiffer, D.; Franke, U.; Forstner, W. Curb reconstruction using conditional random fields. In: Intelligent Vehicles Symposium (IV), 2010 IEEE, 2010, p. 203-210.

S.S. Bueno, H. Azevedo, L. M. E. P. J. R. A. V. J. A. A platform for research and development of outdoor ground robotics (original title in portuguese). In: Simposio Brasileiro de Automacao Inteligente, 2009, p. 1 - 6.

Takahashi, A.; Ninomiya, Y.; Ohta, M.; TAnge, K. A robust lane detection using real-time voting processor. In: Intelligent Transportation Systems, 1999. Proceedings. 1999 IEEE/IEEJ/JSAI International Conference on, 1999, p. 577 -580.

Talukder, A.; Manduchi, R.; Rankin, A.; Matthies, L. Fast and reliable obstacle detection and segmentation for cross-country navigation. In: Intelligent Vehicle Symposium, 2002. IEEE, 2002, p. 610-618 vol.2. 
Tan, C.; Hong, T.; Chang, T.; Shneier, M. Color model-based real-time learning for road following. In: Intelligent Transportation Systems Conference, 2006. ITSC 'O6. IEEE, 2006, p. 939-944.

Thorpe, C.; Hebert, M.; Kanade, T.; Shafer, S. Vision and navigation for the carnegie-mellon navlab. Pattern Analysis and Machine Intelligence, IEEE Transactions on, v. 10 , n. 3 , p. $362-373,1988$.

Tukey, J. W. Exploratory data analysis. Addison-Wesley, 1977.

V.B. Sabbagh, E.J.R. Freitas, G. C. M. S. M. B. T. S. P. I. L. T. G. P. Development of a control system for an autonomous car (original text in portuguese). In: Congresso Brasileiro de Automatica, 2010, p. 928 - 933.

VISLAB Artificial vision and intelligent systems laboratory. http://www.vislab.it, visitado em Abril, 2012.

Vitor, G. B.; Victorino, A. C.; Ferreira, J. V. A probabilistic distribution approach for the classification of urban roads in complex environments. In: Workshop on Modelling, Estimation, Perception and Control of All Terrain Mobile Robots on IEEE International Conference on Robotics and Automation (ICRA), 2014.

VOLVO Volvo. http://www.carsuk.net/volvos-drive-worlds-first/ -large-scale-autonomous-driving-pilot-project/, 2015.

WHO World health organization. Road traffic deaths. http://www. who. int/gho/ road_safety/mortality/traffic_deaths_number/en/index.html, 2014.

ZHAO, G.; YUAN, J. Curb detection and tracking using 3d-lidar scanner. In: Image Processing (ICIP), 2012 19th IEEE International Conference on, 2012, p. 437-440.

Ziegler, J.; Bender, P.; Schreiber, M.; Lategahn, H.; Strauss, T.; Stiller, C.; Dang, T.; Franke, U.; Appenrodt, N.; Keller, C.; Kaus, E.; Herrtwich, R.; Rabe, C.; Pfeiffer, D.; Lindner, F.; Stein, F.; Erbs, F.; EnZWeiler, M.; KNÖPPel, C.; Hipp, J.; Haueis, M.; Trepte, M.; Brenk, C.; Tamke, A.; GhanaAt, M.; Braun, M.; Joos, A.; Fritz, H.; Mock, H.; Hein, M.; ZeEb, E. Making bertha drive: An autonomous journey on a historic route. Intelligent Transportation Systems Magazine, IEEE, v. 6, n. 2, p. 8-20, 2014. 
Zoccoler, M.; Shinzato, P. Y.; HatA, A. Y.; Wolf, D. F. Automatic semantic waypoint mapping applied to autonomous vehicles. In: Robotic Symposium, 2014. LARS 14. IEE Latin American, 2014. 

APPENDIX

\section{$A$}

Published Papers

\section{A.1 Journal Papers}

1. Shinzato, P. Y. ; Gomes, D. ; Wolf, D. F. . Graph-Based Estimation of Obstacles and Road Area with Non-Accurate and Sparse 3D Points. IEEE Intelligent Transportation Systems Transactions. (SUBMITTED)

2. FERNANDES, LEANDRO C. ; Souza, Jefferson R. ; PESSIN, GUSTAVO ; SHINZATO, PATRICK Y. ; SALES, DANIEL ; MENDES, CAIO ; PRADO, MARCOS ; KLASER, RAFAEL ; MAGALHÃES, ANDRÉ CHAVES ; HATA, ALBERTO ; PIGATTO, DANIEL ; BRANCO, KALINKA CASTELO ; GRASSI, VALDIR ; OSORIO, FERNANDO S. ; WOLF, DENIS F. . CaRINA Intelligent Robotic Car: Architectural Design and Applications. Journal of Systems Architecture, v. 1, p. 1-21, 2014.

3. Souza, J. R. ; PESSIN, G. ; Shinzato, P. Y. ; OSORIO, F. S. ; Wolf, D. F. . Vision-based Waypoint Following Using Templates and Artificial Neural Networks. Neurocomputing (Amsterdam), v. 107, p. 77-86, 2013.

4. Shinzato, P. Y. ; Wolf, D. F. . A Road Following Approach Using Artificial Neural Networks Combinations. Journal of Intelligent \& Robotic Systems, v. 62, p. 527-546, 2011. 
5. Souza, J. R. ; SALES, D. O. ; Shinzato, P. Y. ; OSORIO, F. S. ; Wolf, D. F. . Template-based autonomous navigation and obstacle avoidance in urban environments. Applied Computing Review, v. 11, p. 49-59, 2011.

\section{A.2 Conference Papers}

1. Shinzato, P. Y. ; Gomes, D. ; Wolf, D. F. . Road Estimation with Sparse 3D Points From Stereo Data. In: International IEEE Conference on Intelligent Transportation Systems - ITSC, 2014, Qingdao, China. IEEE Intelligent Transportation Systems - ITSC, 2014, China.

2. Zocooler, M.; Shinzato, P. Y.; Hata, A. Y.; Wolf, D. F. . Automatic Semantic Waypoint Mapping Applied to Autonomous Vehicles. In: Latin American Robotics Symposium, 2014, São Carlos. IEEE Latin American Robotics Symposium, 2014.

3. Batista, M. P.; Shinzato, P. Y.; Gomes, D.; Wolf, D. F. . Lane detection and estimation using Perspective Image. In: Latin American Robotics Symposium, 2014, São Carlos. IEEE Latin American Robotics Symposium, 2014.

4. Shinzato, P. Y. ; Wolf, D. F. ; Stiller, C. . Road Terrain Detection: Avoiding Common Obstacle Detection Assumptions Using Sensor Fusion. In: IEEE Intelligent Vehicles Symposium - IV, 2014, Dearborn. IEEE Intelligent Vehicles Symposium - IV, 2014.

5. MENDES, C. C. ; Shinzato, P. Y. ; OSORIO, F. S. ; Wolf, D. F. . Detecção de Obstáculos em Tempo Real para Sensores de Profundidade Utilizando uma GPU. In: Congresso Brasileiro de Automática - CBA, 2012, Campina Grande. Congresso Brasileiro de Automática - CBA, 2012. p. 1-8.

6. Shinzato, P. Y. ; GRASSI JR., V. ; OSORIO, F. S. ; Wolf, D. F. . Fast visual road recognition and horizon detection using multiple artificial neural networks. In: IEEE Intelligent Vehicles Symposium - IV, 2012, Alcala de Henares. IEEE Intelligent Vehicles Symposium - IV, 2012. p. 1090-1095.

7. FERNANDES, L. C. ; Souza, J. R. ; Shinzato, P. Y. ; MENDES, C. C. ; OSORIO, F. S. ; Wolf, D. F. ; PESSIN, G. . Intelligent Robotic Car for Autonomous Navigation: Platform and System Architecture. In: Brazilian Conference on Critical Embedded Systems CBSEC, 2012, Campinas. Brazilian Conference on Critical Embedded Systems -CBSEC, 2012. p. 12-17. 
8. Shinzato, P. Y. ; Osório, F. S. ; Wolf, D. F. . Visual Road Recognition Based in Multiple Artificial Neural Networks. In: Conferência Brasileira de Sistemas Embarcados Críticos, 2011, São Carlos. Conferência Brasileira de Sistemas Embarcados Críticos, 2011. p. 26-31.

9. Souza, J. R. ; PESSIN, G. ; Shinzato, P. Y. ; Osório, F. S. ; Wolf, D. F. . Vision-based Autonomous Navigation Using Neural Networks and Templates in Urban Environments. In: Conferência Brasileira de Sistemas Embarcados Críticos, 2011, São Carlos. Conferência Brasileira de Sistemas Embarcados Críticos, 2011. p. 55-60.

10. Souza, J. R. ; SALES, D. O. ; Shinzato, P. Y. ; Osório, F. S. ; Wolf, D. F. . Template-based autonomous navigation in urban environments. In: ACM Symposium on Applied Computing, 2011, Taichung. ACM Symposium on Applied Computing, 2011. p. 1381-1386.

11. Shinzato, P. Y. ; Osório, F. S. ; Wolf, D. F. . Visual Road Recognition Using Artificial Neural Networks and Stereo Vision. In: IEEE/RSJ International Conference on Intelligent Robots and Systems - IROS - Workshop on Visual Control of Mobile Robots, 2011, San Francisco. IEEE/RSJ International Conference on Intelligent Robots and Systems IROS - Workshop on Visual Control of Mobile Robots, 2011. p. 13-18.

12. Shinzato, P. Y. ; Osório, F. S. ; MENDES, C. C. T. ; Wolf, D. F. . Performance Evaluation of Different Machine Learning Techniques With Stereo Vision Used to Road Detection Task. In: Congresso Brasileiro de Inteligência Computacional - CBIC, 2011, Fortaleza. Congresso Brasileiro de Inteligência Computacional - CBIC, 2011. p. 1-6.

13. EBOLI, G. B. ; Shinzato, P. Y. ; Wolf, D. F. . Análise de Atributos de Imagem em Segmentação Baseada em Blocos Utilizando Redes Neurais Artificiais. In: Congresso Brasileiro de Inteligência Computacional - CBIC, 2011, Fortaleza. Congresso Brasileiro de Inteligência Computacional - CBIC, 2011. p. 1-6. 